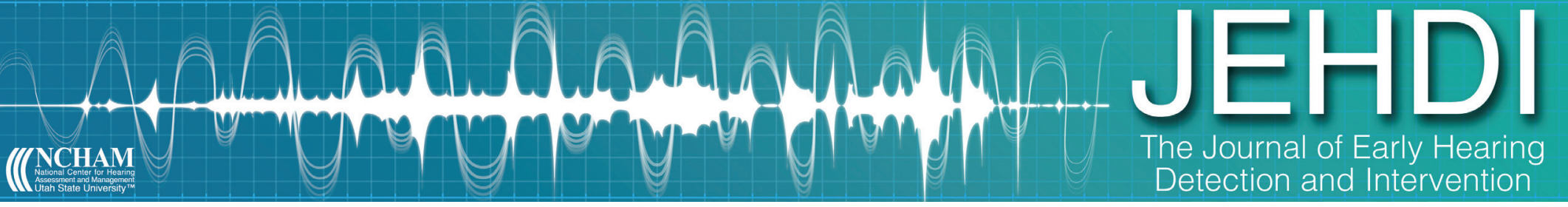

\title{
Year 2019 Position Statement: Principles and Guidelines for Early Hearing Detection and Intervention Programs
}

The Joint Committee on Infant Hearing

\section{Table of Contents}

Executive Summary.

Background

Principles

Guidelines for Early Hearing Detection and Intervention Programs.

Newborn Hearing Screening Programs.......

Pediatric Diagnostic Audiology.

Medical Evaluation

Early Intervention: Services for Infants/

Toddlers from Birth to Age Three Years and Their Families.

Medical Home and Ongoing Surveillance....

Protecting the Rights of Infants/Toddlers and Families.

EHDI Information Technology Infrastructure

Benchmarks and Quality Indicators.

Future Directions.

Research Needs

Acknowledgments.

References.

\section{Abbreviations}

2 AAA - American Academy of Audiology

AABR - Automated Auditory Brainstem Response

AAP - American Academy of Pediatrics

ABA - American Board of Audiology

ABR - Auditory Brainstem Response

$4 \mathrm{AHRQ}$ - Agency for Healthcare Research and Quality

ANSI - American National Standards Institute

ASHA - American Speech-Language-Hearing Association

ASL - American Sign Language

4 ASSR - Automated Steady-State Response

BOA - Behavioral Observation Audiometry

CART - Communication Access Real-time Translation

5 CAEP - Cortical Auditory Evoked Potentials

CMV - Cytomegalovirus

cCMV - Congenital Cytomegalovirus

$12 \mathrm{CDC}$ - Centers for Disease Control and Prevention

CE - Click Evoked

$17 \mathrm{Cl}$-Cochlear Implant

$\mathrm{dB}$ - Decibel

DEC - Division of Early Childhood

DPOAE - Distortion Product Otoacoustic Emissions

DSL - Desired Sensation Level

24 ECMO - Extracorpeal Membrane Oxygenation

EHDI - Early Hearing Detection and Intervention

EHDI PALS - Early Hearing Detection \& Intervention: Pediatric

28 Audiology Links to Services

$\mathrm{HL}$ - Hearing Level

HRCT - High Resolution Computed Tomography

HRSA - Health Resources and Services Administration

IDEA - Individuals with Disabilities Education Act

IEP - Individualized Education Program

33 IFSP - Individual Family Service Plan

IOM - Institute of Medicine

34 ISO - International Organization for Standardization

JCAHO - Joint Commission on Accreditation of Health Care Organizations

$35 \mathrm{JCIH}-$ Joint Committee on Infant Hearing

LTF/LTD - Loss-to-Follow-Up/Loss-to-Documentation

MRI - Magnetic Resonance Imaging

NCHAM - National Center for Hearing Assessment and Management

NICHQ - National Institute for Children's Health Quality Disorders

NICU - Neonatal Intensive Care Unit

$\mathrm{NIH}$ - National Institutes of Health

NLM - U. S. National Library of Medicine

NQF - National Quality Forum

OAE - Otoacoustic Emissions

PCP - Primary Care Physician

PHI - Public Health Information

RECD - Real-Ear to Coupler Difference

SPL - Sound Pressure Level

TEOAE - Transient Evoked Otoacoustic Emissions

TJC - The Joint Commission

UNHS - Universal Newborn Hearing Screening

VRA - Visual Reinforcement Audiometry

WNL - within normal limits 


\section{Terminology}

In this 2019 Statement, the Joint Committee on Infant Hearing $(\mathrm{JClH})$ seeks to use terms that: (a) are acceptable to a range of stakeholders, and (b) clearly convey the intended meaning to the entire community. Because of the diversity of the committee's composition and represented viewpoints, a compromise resulted in choosing currently-recognized terms that reflect accepted, person-first language. In particular, the term infant or child who is deaf or hard of hearing is intended to be inclusive of the entire spectrum of children, representing varied hearing levels. This spectrum includes children who are deaf or hard of hearing whose hearing losses may be congenital or acquired, unilateral or bilateral, of any degree from minimal to profound, and of any type, including conductive, sensory (sensorineural), auditory neuropathy, and mixed hearing condition, whether permanent, transient, or intermittent. This spectrum includes those individuals who identify themselves as being a part of either, or both, the Deaf or hard-of-hearing communities.

The commonly used term hearing loss is replaced, when grammatically appropriate to the written English language, with the terminology such as hearing thresholds in the mild, moderate, severe, or profound range, acknowledging that for an infant who is born with hearing thresholds outside the typical (normal) range, no loss has actually occurred. The $\mathrm{JCIH}$ recognizes that terms like hearing loss, hearing impairment, and hearing level have different values or interpretations assigned to them depending on one's cultural perspective. It is the intent of the $\mathrm{JCIH}$ to convey audiological concepts using culturally-sensitive language whenever possible. However, there are times the term hearing loss is retained to clearly convey audiological concepts/conditions, including references to late onset and progressive types. Further, use of the word normal as a type of hearing is replaced, when appropriate, with the word typical to avoid any suggestion of the stigma of abnormality. Finally, in an effort to use clear language, the term refer for a hearing screening result that is a not-pass outcome is avoided, due to lack of clarity and confusion about the meaning and implications of the word refer. The term fail, which in years past had been discouraged in the belief that it would stigmatize infants, is recognized as a commonly-used term in the medical world to describe the outcome of a binary screening and has been adopted for use in this document.

\section{Executive Summary}

Early Hearing Detection and Intervention (EHDI) activities beginning at the birth hearing screening and culminating in early intervention, have positively impacted outcomes for children who are deaf or hard of hearing and their families in the United States and world-wide. Universal newborn hearing screening has resulted in significantly lowering the average age of identification. Screening is a necessary first step, but does not ensure the next critical steps of timely identification and diagnosis of children who are deaf or hard of hearing, amplification, and referral to early intervention, all with the goal of promoting language development.

The goal of EHDI is to assure that all infants are identified as early as possible, and appropriate intervention initiated, no later than 3-6 months of age. There is a body of literature which demonstrates that children and families experience optimal outcomes when these benchmarks are met. Additionally, communication and linguistic competence (in spoken language, signed language, or both) are achievable when timelines are met, and when optimal audiologic and early intervention services are accessible. There remain critical areas of improvement within the EHDI system to ensure newborns benefit from early recognition and have access to appropriate supports.

This current 2019 document builds on prior Joint Committee on Infant Hearing ( $\mathrm{JClH}$ ) publications (2013 $\mathrm{JCIH}$ supplement on Early Intervention and $2007 \mathrm{JCIH}$ Guidelines), updating best practices through literature reviews and expert consensus opinion on screening; identification; and audiological, medical, and educational management of infants and young children and their families.

The current $\mathrm{JClH}$ document includes the following highlights.

\section{Global Benchmarks and Rationale}

- A review and reminder of the importance of early diagnosis of hearing loss following best-practices.
- Recognition of the value of implementation standards for EHDI information systems.

- Recognition of the frequency, and impact, of delayed-onset and/or progressive hearing loss in infants and the need for continued surveillance of auditory and speech-language development in all infants, regardless of outcome of newborn hearing screening.

- States who meet the 1-3-6 benchmark (screening completed by 1 month, audiologic diagnosis by 3 months, enrollment in early intervention by 6 months) should strive to meet a 1-2-3 month timeline.

\section{Newborn Screening}

- Endorsement of the necessity for audiology oversight of hearing screening programs.

- Recognition of the critical need for the ability to calibrate screening equipment using a uniform and validated standard across all screening devices.

- Recognition of the need for manufacturers of screening equipment to provide data on the proportion of children who are deaf or hard of hearing who pass the screening but are subsequently found to have a variety of degrees and types of hearing loss.

- An endorsement, for well-born infants only, who are screened by automated auditory brainstem response (AABR) and do not pass, that rescreening and passing by otoacoustic emissions testing is acceptable, given the very low incidence of auditory neuropathy in this population.

- An endorsement of rescreening in the medical home in some circumstances. If the rescreening is performed in the provider's office, the provider is responsible for reporting results to the state EHDI program.

\section{Diagnostic Audiology and Audiological Interventions}

- A review of current research on the physiologic/ electrophysiologic methods for diagnostic audiologic evaluation of hearing in infants.

- A reaffirmation of the importance of fitting hearing aid amplification using objective, evidence-based protocols to ensure maximal audibility. 


\section{Early Intervention and Family Support}

- Reaffirmation of the need to provide families with individualized support and information specific to language and communication development to support children who are deaf or hard of hearing by providing exposure to language models at the earliest possible age to ensure optimal cognitive, emotional, and educational development.

- Recognition that some families may benefit from infant mental health supports. Infant mental health is a field of research and practice that focuses on optimizing social, emotional, behavioral, and cognitive development of infants in the context of the emerging relationships between parents and infants.

\section{Medical Considerations}

- Reaffirmation of the need for otologic/medical evaluation and management of the newly-identified infant to be carried out as soon as possible following confirmation, in an effort to address potentially reversible conditions, discover associated medical disorders that can impact the infant's general health, and identify conditions that can impact communication strategy choice.

- Recognition that Congenital Cytomegalovirus has a larger impact than previously recognized.

- Updated risk indicators for congenital hearing conditions, including a new table with specified intervals for audiologic evaluation.

- Consideration of reduction in the FDA-approved age for cochlear implantation to less than 12 months.

JCIH's guiding principle is for continued improvements in the EHDI system. This includes lowering the age of identification and diagnosis of infants, as well as ensuring timely and effective interventions to improve language and social-emotional outcomes in children who are deaf or hard of hearing. Amplification (hearing aids, cochlear implants, bone conduction aids) and early language interventions (whether signed language, spoken language or both) should be based on best practice protocols and evidence-based practice as soon as possible following a diagnosis of hearing loss.

The Joint Committee on Infant Hearing $(\mathrm{JCIH})$ endorses early detection and early intervention for all infants who are, or who are at risk of being or becoming, deaf or hard of hearing. The goals of early hearing detection and intervention (EHDI) are to maximize language and communication competence, literacy development, and psychosocial well-being for children who are deaf or hard of hearing. Without appropriate language exposure and access, these children will fall behind their hearing peers in communication, language, speech, cognition, reading, and social-emotional development, and delays may continue to affect the child's life into adulthood. With early detection and appropriate, targeted intervention, developmental milestones for an infant who is deaf or hard of hearing can be expected to be achieved, more accurately reflecting the child's true potential (Tomblin, Oleson, Ambrose, Walker, \& Moeller, 2014; Yoshinaga-Itano, Baca, \& Sedey, 2010). Focusing on the importance of prompt diagnosis and timely, high-quality early intervention for such infants, EHDI systems should facilitate seamless transitions for infants and their families through the processes of screening, audiologic and medical diagnosis, and intervention.

\section{Background}

In the absence of early recognition (and with the resulting lack of access to language), a child who is deaf or hard of hearing in infancy, can experience delays in speech and language development, academic achievement, and social and emotional outcomes. Historically, children who are deaf or hard of hearing were not identified until two-to-three years of age, and those with hearing thresholds between 25 and $40 \mathrm{~dB}$ hearing level $(\mathrm{HL})$ were often undetected until school age (Yoshinaga-Itano, Sedey, Coulter, \& Mehl, 1998).

In the late 1980s, federal agencies such as the United States (U.S.) Health Resources and Services Administration (HRSA) and the National Institute on Deafness and Other Communication Disorders (NIDCD)/ National Institutes of Health $(\mathrm{NIH})$ recognized the new and evolving technologies for screening hearing in newborns, and appreciated the potential of universal newborn hearing screening (UNHS) to allow early diagnosis and prompt intervention for infants who were born deaf or hard of hearing (NIH, 1993). Soon thereafter in the 1990s, newborn hearing screening programs became a reality in a number of states. In the new millennium, newborn hearing screening has become the standard of care, not only for U.S. hospitals and birthing centers, but also for many countries around the world (Olusanya, 2011; van Dyk, Swanepoel, \& Hall, 2015).

EHDI programs throughout the nation have demonstrated not only the feasibility of UNHS programs, but also the increasingly measurable benefits of early identification and intervention (Ambrose, Unflat Berry, et al., 2014; Tomblin et al., 2014; Tomblin et al., 2015). Despite on-going challenges to track all infants who do not pass initial hearing screening, and despite the resulting delays in diagnosis and shortcomings in assuring adequate follow-up, both the average age of diagnosis and the average age of referral to early intervention have steadily decreased (Holte et al., 2012). As a result, each year in the United States some 5,000 infants who are deaf or hard of hearing and their families have the opportunity to experience improved outcomes in the child's language development (through early access to spoken and/or signed language), as well as improved outcomes in the child's overall well-being (Williams, Alam, \& Gaffney, 2015). 


\section{Principles}

All identified and at-risk children and their families should have access to resources necessary to reach their maximum potential. The following principles provide the foundation for effective EHDI systems and have been updated, building on and expanding the practices stipulated and endorsed in the $\mathrm{JCIH} 2007$ position statement.

\section{EHDI 1-3-6 Goals}

1. All infants should undergo hearing screening prior to discharge from the birth hospital and no later than one month of age, using physiologic measures with objective determination of outcome.

2. All infants whose initial birth-screen and any subsequent rescreening warrant additional testing should have appropriate audiologic evaluation to confirm the infant's hearing status no later than 3 months of age.

3. A concurrent or immediate comprehensive otologic evaluation should occur for infants who are confirmed to be deaf or hard of hearing.

4. All infants who are deaf or hard of hearing in one or both ears should be referred immediately to early intervention in order to receive targeted and appropriate services.

5. A simplified, coordinated point of entry into an intervention system appropriate for identified children is optimal.

6. Early intervention services should be offered through an approach that reflects the family's preferences and goals for their child, and should begin as soon as possible after diagnosis but no later than six months of age and require a signed Part $C$ of IDEA (Individuals with Disabilities Education Act, 2004) Individualized Family Service Plan.

7. The child and family should have immediate access, through their audiologist, to high-quality, well-fitted, and optimized hearing aid technology. Access should also be assured, depending on the child's needs, to cochlear implants $(\mathrm{Cl})$, hearing assistive technologies, and visual alerting and informational devices.

\section{Updated Principles Beyond EHDI 1-3-6}

8. The EHDI system should be family-centered with infant and family rights and privacy guaranteed through informed and shared decision-making, and family consent in accordance with state and federal guidelines.

9. Families should have access to information about all resources and programs for intervention, and support and counseling regarding the child's educational and communication/language needs.

10. All infants and children, regardless of newborn hearing screening outcome, should be monitored within the medical home according to the periodicity tables regarding their communication development (American Academy of Pediatrics [AAP] Committee, 2017).
11. Professionals with appropriate training should provide ongoing surveillance of communication development to all children with or without risk indicators.

12. Appropriate interdisciplinary early intervention programs for identified infants and their families should be provided by professionals knowledgeable about the needs and requirements of children who are deaf or hard of hearing $(\mathrm{JClH}, 2013)$.

13. Early intervention programs should recognize evidence-based practices and build on strengths, informed choices, language traditions, and cultural beliefs of families they serve.

14. EHDI information systems should be designed and implemented to interface with clinical electronic health records and population-based information systems to allow the exchange of electronic health information for the purposes of outcome measurement, quality improvement, and reporting the effectiveness of EHDI services for the patient/family within the medical home, healthcare community, state, and federal levels.

\section{Guidelines for Early Hearing Detection and Intervention Programs}

The guidelines presented in this 2019 position statement were developed to update the $2007 \mathrm{JClH}$ position statement and serve to support the goals of universal access to hearing screening, evaluation, and intervention for newborns and infants. The guidelines provide current information on the development and implementation of successful EHDI systems. Studies have demonstrated that current screening technologies are effective in identifying hearing thresholds of approximately 35-40 dB $\mathrm{HL}$ and greater (Norton et al., 2000a); however, mildly elevated hearing thresholds are not identified using current screening technologies and even mildly elevated hearing thresholds can impact speech and language development (Walker et al., 2015). Language, speech, social-emotional, and academic development may be affected when children who are deaf or hard of hearing lack full or complete access to linguistic input (Dillon, Cowan, \& Ching, 2013; Robertson, Howarth, Bork, \& Dinu, 2009; Yoshinaga-Itano, et al., 1998). Specific attention is given in this document to infants who receive care in the neonatal intensive care unit (NICU) because research data indicate that this population is at higher risk for hearing loss (Moeller, Carr, Seaver, Stredler-Brown, \& Holzinger, 2013).

Regardless of previous hearing-screening outcomes, all infants with or without risk factors should receive ongoing surveillance of communicative development beginning at 2 months of age during well-child visits in the medical home (AAP Committee, 2017). This recommendation provides an alternative, more inclusive strategy of surveillance of all children within the medical home based on the pediatric periodicity schedule (AAP Committee, 2017). All infants who do not pass the speech-language portion of a developmental screening in the medical home or for whom 
there is a concern regarding hearing or language should be referred for speech-language evaluation and audiology assessment. This protocol permits the detection of children with either missed neonatal or delayed-onset hearing loss, irrespective of the presence or absence of a high-risk indicator.

Depending on the screening technology selected, infants with auditory neuropathy may not be detected through a UNHS program. Given the low incidence of auditory neuropathy in the well-baby nursery, the $\mathrm{JCIH}$ recommends the use of either automated auditory brainstem response (AABR), or otoacoustic emissions (OAEs), or both for initial screenings and/or rescreening. However, the $\mathrm{JCIH}$ continues to recommend AABR screening and rescreening protocols in the NICU to allow for detection of auditory neuropathy.

Optimal EHDI programs have been defined as meeting the EHDI 1-3-6 goals. To provide appropriate access to language stimulation and intervention services as soon as possible, EHDI programs meeting current targets might consider setting a new target of 1-2-3 months (screening completed by one month of age, audiologic diagnosis completed by two months of age, and early intervention initiated no later than three months of age). The earliest possible age of identification is encouraged for two reasons. First, the infant can receive earlier intervention for auditory and/or visual access to language. Second, objective audiologic testing can be completed without sedation during the natural sleep that occurs when newborn/infants are young enough to sleep for prolonged periods of time. It may not be appropriate to apply this timeline to infants receiving care in the NICU. Because the majority of very preterm infants may still be in the NICU at 3 months of age, a recommendation is made that for very preterm infants with prolonged hospitalization, a diagnostic audiologic evaluation prior to discharge from the NICU be completed. Infants identified as being deaf or hard of hearing could then be referred directly for early intervention and audiological follow-up services at the time of discharge.

Primary care physicians need to be aware of the necessity for monitoring communication and language skills according to the most recent developmental periodicity tables (AAP Committee, 2017). Any child who demonstrates delayed auditory and/or communication skills development, even if that child passed newborn hearing screening, should be promptly referred for audiologic evaluation. Additionally, any child whose parents/caregivers express concern regarding auditory and/or communication skills development, even if that child passed newborn hearing screening, should be promptly referred for audiologic evaluation.

\section{Newborn Hearing Screening Programs}

Multidisciplinary teams of professionals including audiologists, physicians, and nursing personnel are needed to establish the UNHS component of EHDI programs (JClH, 2013; Moeller et al., 2013). An audiologist with experience in evaluating newborns and young children should be involved in the development and oversight of each component of the hearing screening program, particularly at the level of statewide implementation and, whenever possible, at the individual hospital level. Hospitals and agencies should also designate a physician/provider to oversee the medical aspects of the EHDI program.

Each team of professionals responsible for the hospital-based UNHS program should review the hospital infrastructure in relationship to the screening program. Evidence-based research is needed to support the reliability and validity of all screening equipment used, whether OAE or AABR. Hospital-based programs must consider a variety of issues, including the population of infants to be screened, screening technology choices, validity of the specific screening device, screening protocols (including the timing of screening relative to hospital discharge), availability of qualified screening personnel, suitability of the acoustical and electrical environments, follow-up referral criteria and pathways, information management, and quality control and improvement. Reporting and communication protocols must be well-defined, and such protocols must include the content of reports to physicians and to families, documentation of results in medical records, flow of screening result information from hospital-based medical records to outpatient records, and methods for reporting to state registries and national data sets. Hospitals should identify and define the referral pathway and systems for ensuring that each baby who does not pass in-patient screening has access to a pediatric audiologist or certified/registered screener who can complete the outpatient rescreening, and when indicated, refer for or complete a diagnostic audiologic evaluation according to the EHDI $1-3-6$ or $1-2-3$ goals.

\section{Audiology Oversight of Newborn Hearing Screening Programs}

Audiology oversight is recommended for all state/territory hearing screening programs, both at the systems level and at the individual program level. The systemsoversight audiologist (in collaboration with medical and nursing personnel) should design and implement procedures to (a) conduct periodic on-site and/or remote surveillance (e.g., phone, email, and web-conference) of the individual hospital programs, (b) provide oversight and participate in the writing of the policies and procedures for screening, (c) train staff and assure competency, and (d) monitor program statistics and quality assurance. Referral pathways and timelines should be developed in conjunction with community resources and the state EHDI program to ensure timely rescreening of those infants who do not pass their birth hearing screens, as well as timely and appropriate diagnostic audiologic evaluations.

Across the fifty states and nine territories, different models exist for audiology oversight of an individual UNHS program. In some state EHDI systems, individual hospital 
programs contract with an outside screening entity, an audiologist, or audiology program, while others provide this oversight from within their own staff audiology personnel. Some systems provide oversight over a specific geographic region. An audiologist with requisite knowledge should oversee all aspects of each individual UNHS program. This should entail, at a minimum:

1. Selection of screening technology and equipment based on the population(s) to be screened.

- Consideration of whether the equipment will be used for well babies, infants receiving care in the NICU, or both (e.g., due to the maturation of the auditory neural pathway, pre-term infants and/or older infants may not meet the criteria for automated screening protocols whose pass/fail outcome is based on average responses from a term baby).

- Comprehension of the manufacturer's required age-limits for the specific screening equipment selected to conduct valid screening of premature, as well as older infants.

- Referral of an infant who is too old for screening using automated equipment according to the manufacturer's validation statement to a diagnostic audiologic evaluation if appropriate (e.g., if the infant is in the NICU, the diagnostic evaluation can be conducted as an in-patient procedure).

2. Confirmation that initial equipment calibration performed by the manufacturer or distributor is done in a manner consistent with purported screening parameters.

- Establishment of a method by which an independent entity (e.g., hospital clinical engineering, local special instrument distributors who conduct routine annual audiometric calibration of all equipment, etc.) can perform calibration or provide oversight to ensure that equipment parameters remain stable and appropriate.

- Calibration of test stimuli performed in a coupler appropriate to the transducer and in reference to a published calibration standard (American National Standards Institute [ANSI; http://www.ansi.org], or International Organization for Standardization [ISO; http://www.iso.org/iso/home/standards.htm]).

- Calibration procedures should be fully transparent to the end-users, including such metrics as stimulus parameters (i.e., intensity and bandwidth of the stimulus, relationship of stimulus metrics to hearing threshold estimates as a binary outcome of pass/ fail, and expected pass/fail metrics for both the well-baby and NICU populations).

- Requirements for troubleshooting, annual calibration, and expected performance in the population to be screened should be reviewed by the audiologist.

3. Development and implementation of protocols for training and certifying competence of new screeners.

- Regular educational in-service training for all program personnel should be an ongoing commitment to assess and ensure continued competence.
- The audiologist, along with the manufacturer, should provide training to the screening personnel in the correct use of the screening equipment.

- The audiologist should provide training to screeners in optimizing infant state for screening. This includes ensuring a quiet, preferably sleeping baby, and using techniques to verify that the ear canal is open and patent to the extent possible before the earphone or probe is placed.

- The audiologist may, depending on the size and needs of the program, conduct a train-the-trainer educational program to produce an adequate and continuing supply of screeners.

4. Development and implementation of policies, procedures, and protocols, in conjunction with nursing, medical, and other personnel (e.g., care coordination), based on best-practices and in accordance with state regulations.

- Identify the screening methods, timing, and equipment to be used to conduct the screening, including provisions for readily available backup equipment in the event of an equipment malfunction.

- Provide samples or scripts of information (spoken, signed, and/or written in the language of the home) given to the family regarding the screening outcome, and including: (a) a definition of the term pass and a definition for the word fail (or did not pass, or refer, alternate terms that are sometimes used for a non-passing result); (b) the necessity for appropriate follow-up rescreening when indicated; (c) the importance of early and timely completion of such rescreening and diagnostic audiologic evaluations, if indicated; (d) the benefits of early intervention for the infant who is subsequently identified as being deaf or hard of hearing, and (e) a clear statement regarding next steps. When appropriate, information regarding third-party coverage of the screening and audiologic diagnosis costs should be provided. Identify the procedures for communicating to the family that the infant requires additional follow-up testing due to the results of the screening or missed screening. Communication should occur in a manner that is culturally and linguistically appropriate, and should be provided orally for hearing families (with a foreign language interpreter as indicated), as well as in writing or through audio-visual means. Families whose primary language is American Sign Language (ASL) or another sign language, or a language different from English should be provided with an interpreter.

- Provide hearing screening results and outcomes to the infant's medical home or primary care provider and to the state EHDI program.

- Both the family and the primary-care provider should be advised that passing a hearing screening performed either by OAE or by AABR testing does not imply that hearing thresholds are within normal limits (WNL), only that thresholds are not greater than approximately 35-40 dB HL. 
- Clearly communicate that a pass outcome implies that the infant passes both ears simultaneously (in the same screening session). Specifically, an infant who does not pass both ears in the same screening session, even if each ear has separately passed a screening, does not constitute a pass outcome.

Parents should not be advised, "one ear passed and the other ear did not pass."

- Describe the training and supervision of individuals with responsibility to inform the family of screening results, ensuring that the family understands the importance of immediate follow-up when the baby does not pass the newborn hearing screening.

- Provide the family with an appointment for outpatient rescreening at the time of discharge from the birth hospital as best practice to decrease the likelihood of loss-to-follow-up/loss-to-documentation (LTF/LTD).

5. Development and implementation of quality assurance procedures that include monitoring screening statistics.

- Outline the procedures for documenting and transmitting the results of the screening in a manner consistent with the Health Insurance Portability and Accountability Act (HIPAA) and other compliance requirements (see the section titled "Protecting the Rights of Infants/Toddlers and Families" below for an expanded discussion).

- Identify and document written procedures to ensure that an infant who does not pass the hearing screening while still in the hospital (or who never completes hearing screening while at the hospital) will receive a timely initial or follow-up screening as an outpatient.

- Identify the procedures for reporting data on a regular basis as required by the state EHDI program. Data reporting should be accomplished using a secure, web-based data system, with individual infant data being reported by the hospital directly to the state EHDI system.

6. Specification of program staffing requirements and definition of the responsibilities of each staff member: screening nurses and other screening personnel, audiology director, medical director.

7. Development of standard operating procedures that outline processes and steps to follow when reviewing and recording hearing screening status in the discharge plan or transfer plan for all newborn infants. Safeguards should be in place to ensure that infants who are transferred to a different unit or facility and who develop a new risk factor are re-screened prior to discharge.

8. Ensure acceptable, independent, on-site oversight by an audiologist who is either employed by the hospital, or is otherwise independent of the contracted entity in screening programs where services are contracted through an outside entity.

\section{Objective Physiologic Screening Measures}

Objectively-determined physiologic measures must be used to screen newborns and young infants to identify those who may be deaf or hard of hearing (ConeWesson et al., 2000; Johnson et al., 2005a; Norton et al., 2000b). Currently such measures include

automated $O A E$ and auditory brainstem response (ABR). Automated OAE protocols use either transient-evoked OAEs (TEOAE) or distortion-product OAEs (DPOAE). Both automated $O A E$ and $A B R$ technologies provide noninvasive recordings of physiologic activity underlying normal auditory function. Both are easily performed in neonates and infants, and both have been successfully used for UNHS programs (Gravel et al., 2000; Norton et al., 2000b). There are, however, important differences between the two measures. OAE measures a physiologic response from the cochlear outer hair cells, while ABR measurements reflect both cochlear status, as well as auditory neural function extending beyond the cochlea into the brainstem. Thus, the ABR response reflects activity from a greater portion of the auditory pathway than does the OAE. For this reason, ABR will result in a failed outcome when screening infants with auditory neuropathy, whereas screening with OAEs will result in a pass for the baby with auditory neuropathy.

Both OAE and Auditory Brainstem Response (ABR) screening technologies can be used to detect whether the child has a sensory (cochlear) hearing loss/condition (Norton et al., 2000a). However, the results obtained using either technology are affected by concomitant outer ear or middle ear dysfunction that might be present. Consequently, conditions of the outer ear and/or middle ear may result in a failed hearing screening in the presence of normal cochlear and/or neural function. Use of OAE as a screening tool is likely to result in a higher fail rate in the immediate post-birth period as compared with AABR (van Dyk et al., 2015).

Some infants who pass newborn hearing screening will later be identified as deaf or hard of hearing (Johnson et al., 2005b). Although this later-detected hearing loss may reflect delayed-onset or progressive changes in hearing, both AABR and OAE screening technologies will fail to identify children who are deaf or hard of hearing with hearing thresholds that are considered borderline or mild or are in an isolated frequency-region (Young, Reilly, \& Burke, 2011). The hearing threshold detected with AABR screening technology is slightly higher ( 40 to $45 \mathrm{~dB} \mathrm{HL}$ ) as compared with OAE technology ( 30 or $35 \mathrm{~dB} \mathrm{HL}$ ).

Therefore, there is a slightly greater chance of hearing thresholds between 25 and $40 \mathrm{~dB}$ HL going undetected when the initial screening is performed using AABR. Forty-two percent of children who failed a TEOAE screen but did pass an AABR screen were subsequently found to have hearing thresholds greater than $45 \mathrm{~dB} \mathrm{HL}$ (Levit, Himmelfarb, \& Dollberg, 2015). Conversely, OAE technology failed to detect auditory neuropathy of any degree (Johnson, 2005a). 


\section{Interpretive Criteria}

Each hearing screen comprises simultaneous assessment of both the right and left ears. Criteria for hearing screening outcomes should reflect an optimal balance between sensitivity and specificity, considering the prevalence of infant hearing loss (Gorga et al., 2006), manufacturer-reported test performance, and the goal of identifying elevated hearing thresholds that can affect spoken language development. Screening technologies that incorporate automated response detection algorithms were developed to eliminate the need for individual test interpretation, reduce the effects of screener bias or operator error on test outcome, and ensure screening consistency across infants, test conditions, and screening personnel. When statistical probability is used to make pass/fail decisions, as is the case for OAE and AABR screening devices, the likelihood of obtaining a pass outcome by chance alone is increased when screening is performed repeatedly (Type I error). The increased probability of a Type I error must be incorporated into the policies of rescreening, thus $\mathrm{JCIH}$ has renewed the recommendation that (a) no more than two high-quality hospital-based screenings should be performed prior to hospital discharge, and (b) only one high-quality outpatient rescreen be performed prior to referral to a pediatric audiologist for the child who needs follow-up testing after the outpatient rescreen. A high-quality screen implies that the infant is sleeping or resting quietly without movement throughout the screening period, and that patency of the ear canal is assured to the extent possible prior to initiating the screen.

\section{Calibration of Hearing Screening Equipment}

Both screening and diagnostic audiologic measures that are based on electrophysiologic responses employ stimuli for which there is presently no universally-accepted calibration standard. Most current screening technologies use brief-duration stimuli that lack calibration standards set by the American National Standards Institute (ANSI; http:// www.ansi.org). The ISO has a standard that specifies reference threshold sound pressure levels (SPL) and envelopes of standard tonebursts and clicks for test signals of short duration applicable to the calibration of audiometric equipment (http://www.iso.org/iso/home/standards.htm). Calibration of transient stimuli used in infant hearing screening programs is complicated by variability in stimulus parameters (e.g., stimulus, rate, duration) and by types of transducers used in automated devices. Differing transducers and the use of different ear couplers lead to variability in both the stimulus level and spectrum. This is complicated by the fact that there are no universally accepted conversion factors for transforming adult threshold values to infant/pediatric use. Furthermore, while there is general agreement regarding the relationship of electrophysiological threshold measures to subsequent behavioral thresholds within group data, accuracy in predicting behavioral thresholds varies for the individual child (Gorga et al., 2006; McCreery, Kaminski, et al., 2015). In the absence of universally accepted specifications of stimulus level and spectrum for use in newborn hearing screening and infant hearing testing, it is imperative that manufacturers of screening equipment provide objective and transparent calibration information, as well as the pass/fail rates in populations of newborns and infants demonstrated to have normal hearing and those confirmed as deaf or hard of hearing. Even within a single manufacturer, it has been reported that calibration intensities and spectra differed between two devices designed to screen infant hearing, with a significant difference in referral rate that was attributed to these dissimilarities (Hofmann, Luts, Poelmans, \& Wouters, 2012). JCIH called for a calibration standard for newborn screening using ABR in 2007, however, in the United States, there is not yet an ANSI standard.

Finally, it is suggested that, until such time as an ANSI standard is developed, practicable, and promulgated, manufacturers should provide calibration information per the ISO standard that is available in countries outside the United States. The question of false-negative test results (i.e., pass newborn hearing screening when hearing thresholds are elevated) continues to deserve scrutiny. Although it is not the goal of universal newborn hearing screening to identify all infants who are deaf or hard of hearing (e.g., infants with auditory neuropathy will be missed when screened by OAE; infants with minimal-mild hearing thresholds will be missed when either technology is used), the importance of acknowledging, and estimating the occurrence of false-negative outcomes must continue to receive attention. This is particularly critical given the increasingly-recognized significance of even mild threshold elevation in infants and young children (Walker et al., 2015).

\section{Timing of Newborn Hearing Screening}

Infants should have their hearing screened as close to discharge as practicable, while at the same time allowing sufficient time for a single repeat screen to be performed if the infant does not pass the first screen. The second screen, should it be required, should not be performed immediately following the first screen, but should occur at least several hours later. Infants with congenital aural atresia in one or both ears or with visible pinna/ear canal deformity such as stenosis or severe malformation should not be screened in either ear but should be referred for diagnostic audiologic evaluation immediately upon discharge. The diagnostic audiologic evaluation can also be accomplished while the infant is in the NICU or other inpatient hospital unit.

For some infants in the NICU (e.g., infants on ventilators), it may not be feasible or practical to complete a hearing screening prior to one month of age due to the high likelihood of middle ear effusion, noise interference, and electrical interference from equipment. Alternative arrangements should be made for completing the hearing screening on these infants at a time when they are medically stable. 


\section{Screening Protocols in the Well-baby Nursery}

Many inpatient well-baby screening protocols provide one hearing screening and, when necessary, a repeat screening prior to discharge from the hospital, using the same technology with both screenings. Use of either AABR or OAE technology will detect hearing thresholds of children who are deaf or hard of hearing with peripheral conductive and sensorineural etiology, within the limits of the testing threshold of the selected technology (Norton et al., 2000b). When AABR is used as the single screening technology, neural auditory disorders (auditory neuropathy) can also be detected. Some programs use a combination of screening technologies for the well-baby nursery (OAE testing for the initial screening, followed by AABR for rescreening), a so-called two-stage protocol (Lin, Shu, Lee, Lin, \& Lin, 2007). Such a protocol can minimize initial disposable costs and decrease the fail rate at hospital discharge, thereby reducing the subsequent need for outpatient follow-up. Using this approach, infants who fail an OAE screening but subsequently pass an AABR are considered a screening pass.

In the previous $2007 \mathrm{JCIH}$ Statement, it was recommended that infants in the well-baby nursery who do not pass an AABR screening not be rescreened and passed using OAE technology because such infants are presumed to be at risk of having a subsequent diagnosis of auditory neuropathy. At the same time, there is a very low incidence of auditory neuropathy in the well-baby nursery (6-30/100,000 births; Korver, van Zanten,

Meuwese-Jongejeugd, van Straaten, \& Oudesluys-Murphy, 2012). Korver and colleagues note that there is not much evidence available upon which to draw a definitive statistic, and that the evidence that is available is variable. There continues to be an unacceptably high loss-to-follow-up statistic for infants who fail the birth screen and then fail to return for rescreening as an outpatient (Korver et al., 2012). Given these considerations, a new

recommendation is made that screening in the well-baby nursery may be accomplished using either OAE or AABR, with the second (re-) screen (second in-hospital screen), conducted using either technology. The recommendation to rescreen using only AABR technology for the infant who fails initial screening performed with $A A B R$ technology continues to be the Committee's preferred protocol. Less than $1 \%$ of newborns in the well-baby nursery had an AABR fail with an OAE pass as inpatients, and none exhibited this pattern as outpatients (Berg, Prieve, Serpanos, \& Wheaton, 2011). Specifically, re-screening with $O A E$ after failing an AABR is acceptable with the caveat that a baby with auditory neuropathy in the well-baby nursery will be missed using this protocol. In essence, the $\mathrm{JCIH}$ asserts that any pass-screen result of both ears in the same screening session using either technology prior to hospital discharge is acceptable as a passed hearing screen (see "Rescreening in the Outpatient Setting" below for an expanded discussion).

\section{Screening Protocols in the Neonatal Intensive Care Unit}

Infants who have received care in the NICU represent $10 \%$ to $15 \%$ of the newborn population and have been shown to have a higher prevalence of elevated hearing thresholds compared to infants from well-baby nurseries (Robertson et al., 2009; Vohr et al., 2000).

Not only is there a higher prevalence of hearing loss in this population, there is also a higher risk of auditory neuropathy in infants with a history of hyperbilirubinemia and those administered a mycin-class antibiotic (Berg et al., 2005). For this reason, the sole use of AABR for hearing screening is recommended for infants who have received care in the NICU. In addition, infants cared for in the NICU who do not pass the AABR should be

(a) referred directly to an audiologist for rescreening, and

(b) if indicated, given a comprehensive audiological evaluation including diagnostic ABR.

There is no definitive information regarding the intensity of the screening level for AABR equipment. It is known that some infants who pass $A B R$ screening do not pass $O A E$ screening, and that some infants who pass $A B R$ screening subsequently are found to have elevated hearing thresholds. Whether these losses were present at birth or developed later in infancy is not known. There is evidence suggesting that OAE screening is more sensitive to mild hearing thresholds than AABR. In a recent study by Levit and colleagues (2015), 42\% of the infants who failed OAE screening, passed the AABR screening and were subsequently found to have hearing thresholds greater than $45 \mathrm{~dB} \mathrm{HL}$.

The recommendation by $\mathrm{JCIH}$ in 2007 that $\mathrm{AABR}$ is the most appropriate screening technology for infants who received care in the NICU was made based on evidence of the prevalence of neural hearing losses in that population (Berg et al., 2005). There is concern that infants with hearing thresholds less than $40 \mathrm{~dB} \mathrm{HL}$ (common screening intensity of AABR in the NICU) might be at higher risk to be missed than those screened by OAE technology (commonly used in well-baby nurseries). For example, Johnson and colleagues (2005a) demonstrated that as many as one in five newborns with hearing thresholds between 25 and $40 \mathrm{~dB} \mathrm{HL}$ using behavioral testing at age nine months would have potentially passed AABR screening as a newborn due to the fact that their later-demonstrated hearing levels did not rise to the $40 \mathrm{~dB} \mathrm{HL}$ threshold of detection for this screening technology. Similarly, Wood, Davis, and Sutton (2013) found that one significant risk factor associated with delayed-onset hearing loss could be defined as the infant cared for in the NICU who did not pass OAE screening in both ears, but subsequently passed rescreening in both ears when performed with AABR technology. Finally, Berg and colleagues (2005) recommended using AABR followed by $O A E$ screening to screen infants receiving care in the 
NICU. Although the $\mathrm{JCIH}$ has not changed the existing recommendation, screening with both technologies would ensure that children with hearing thresholds in the mild/ moderate range would not be missed in the NICU.

Although use of both technologies in the NICU has advantages and would detect both auditory neuropathy and minimal-mild hearing thresholds with higher rates of identification, there are a number of other factors to be considered. First, there is an increased rate of transient middle ear fluid among high-risk infants cared for in the NICU (Hunter, Prieve, Kei, \& Sanford, 2013). This finding could result in a higher screen refer rate for diagnostic testing. In addition, the change in $\mathrm{JClH}$ recommendations would require hospitals to purchase and monitor two different pieces of equipment (although combination devices have now come on the market), require additional screener time, and impose higher costs on the hospital and insurance. It could also mean additional stress for families of infants with a transient conductive component and a need for unnecessary diagnostic testing. The objective of the current recommendation for continued surveillance of both hearing skills and language development in the medical home is to increase the probability of identifying mild, progressive, and late onset hearing loss (AAP Committee, 2017). However, some infants will regrettably continue to be identified late (Holte et al., 2012). States and hospitals will need to consider the options and the costs in choosing the best technology and protocols to implement.

\section{Communication and Documentation of Results}

Screening results should be conveyed immediately to the family so that they can understand the outcome of the screening and the importance of follow-up when indicated. The use of the scripts developed by the state EHDI program or the National Center for Hearing Assessment and Management (NCHAM) is recommended (NCHAM, n.d.). For the baby who does not pass the hospital screen, the family should be informed in a manner that maximizes the likelihood that follow-up will occur when needed, a manner not overly stressful for the family, but at the same time not suggesting overly-optimistic estimates of the reasons for the failed outcome. To facilitate this process for families, primary health care professionals should work with EHDI team members to ensure that:

- Communications with families are confidential and presented in a caring and sensitive manner, preferably face-to-face.

- Educational materials are offered to families to provide accurate information in an appropriate reading level and in a language the families/caregivers are able to comprehend with clearly-stated next steps (Nicholson et al., 2016).

- Families are informed in a culturally-sensitive and understandable manner that their infant needs further hearing testing, and families are informed about the importance of prompt follow-up (DesGeorges, 2017).

- Before discharge, an appointment should be made for follow-up rescreening or for audiological testing.
- Alternate contact information for a family should be obtained prior to discharge to ensure timely follow-up (Winston-Gerson \& Hoffman, 2017).

Unfortunately, about one third of the time, infants who fail their birth screen do not receive timely and appropriate follow-up (Centers for Disease Control and Prevention [CDC], n.d.-a; Holte et al., 2012). Accordingly, reducing and preventing LTF/LTD starts with birth-hospital hearing screening personnel and training. To facilitate the screening process for primary care providers and better ensure timely follow-up, EHDI systems should ensure that medical professionals:

- Receive the results of the screening test (pass, fail, or not completed) and the type of test administered (OAE or AABR), as documented in the hospital medical record. Although the type of screening test performed has not typically been included in the report to the primary care physician, including this information is now recommended by the $\mathrm{JCIH}$.

- Receive communication directly from the hospital for each infant who does not pass the newborn screening or leaves the hospital unscreened, with any pertinent recommendations for follow-up included in the communication.

\section{Rescreening in the Outpatient Setting}

A critical function in the success of UNHS is the ability to ensure timely retesting for all infants who do not pass their initial (birth) hearing screening. Outpatient rescreening should be performed as soon as possible after hospital discharge, and always before one month of age (or, in the case of an older infant, as soon as possible following discharge). The practice of multiple rescreenings in the outpatient setting delays referral for diagnostic evaluation and impacts timely diagnosis (White, Nelson, \& Muñoz 2016). Rescreening should comprise a single valid rescreen of both ears in the same session, regardless of initial screening results, to ensure that fluctuation or progression in hearing levels are not missed. Due to the fact that hearing threshold levels between 25 and $40 \mathrm{~dB}$ $\mathrm{HL}$ could be present at or near the threshold of the technology being used, the outpatient rescreening should always include the testing of both ears, even if only one ear did not pass the inpatient screening. The outpatient rescreen may be conducted by the birth hospital, by a pediatric audiologist in the community, in the baby's medical home, or in an agency or clinic that adheres to best-practice protocols with appropriate screening equipment and demonstrable screener training and competence. Regardless of who performs the rescreening or the location in which the rescreening is performed, there is an obligation to report outcomes of all rescreening results whether pass or fail, to the state EHDI program.

If the infant does not pass in one or both ears on the rescreen, immediate referral to a pediatric audiologist with capabilities for a diagnostic ABR testing should be made. Preferably, the parent/guardian should be provided 
with an appointment with the audiologist prior to leaving the rescreening facility. When the outpatient rescreen is performed by a pediatric audiologist, and the infant does not pass the rescreen in one or both ears, it is preferable that the diagnostic evaluation be initiated immediately (i.e., during the same appointment).

Outpatient hearing screening at no later than one month of age should also be made available to infants who were discharged before receiving the birth admission screening, and to infants who were born outside a hospital or birthing center in a location that does not provide newborn hearing screening. Similarly, outpatient screening also should be made available to the infant whose parents previously declined or were unable to complete the hearing screening and subsequently decided to have their baby screened.

\section{Rescreening in the Medical Home}

Since the publication of the JCIH 2007 statement, an increasing likelihood of OAE rescreening in the physician's office has been noted (Nelson, Bougatsos, \& Nygren, 2008). Some primary care physicians have OAE screening devices for use in the medical office, both for the purpose of rescreening newborns (when indicated) and for screening older children. The American Academy of Pediatrics has published guidelines regarding rescreening in the medical home (American Academy of Pediatrics, 2014a), and JCIH supports these guidelines. Specifically, the guidelines for rescreening hearing, when performed in the physician's office, include the following highlights.

- Rescreening of infants must be performed using an automated physiologic measurement (OAE or AABR), not by assessing behavioral responses to environmental sounds or noises (e.g., using whispered speech or noisemakers).

- Physicians who rescreen in the medical office are obligated to report rescreen outcomes (both pass and fail results) to the state EHDI system.

- The equipment used for rescreening must be calibrated, and annually re-calibrated, by the manufacturer or other entity (e.g., special-instruments distributor or hospital clinical engineering department).

- There must be a quiet environment for office-based testing to avoid having children fail the rescreening even if they have normal hearing.

- Office-based personnel who perform the rescreening must be appropriately trained in the use of the equipment.

- Infants who were hospitalized in the NICU and who did not pass a hospital-based screening should be referred directly to a pediatric audiologist and not rescreened in the medical home, due to the increased likelihood of hearing loss including auditory neuropathy (American Academy of Pediatrics, 2014a).

- At the time of rescreening, both ears should be tested, even if only one ear did not pass the screening performed at the hospital.
Hospital based UNHS programs have proven efficacy due to the ability to standardize processes and procedures through the state EHDI programs, although, some hospitals provide rescreening and some do not. A shortcoming of most hospital record-keeping entries is that the technology used at the time of hospital-based screening is often not recorded in the hospital discharge summary, and the primary care provider may have some difficulty obtaining such information. However, some state database systems are now designed or are being expanded to require identification of the type of equipment used for the birth screen. JCIH does not support providers performing the initial newborn hearing screening in the office, but rather supports the positions summarized that follow, as outlined by a number of AAP publications (AAP, 2014a, 2014b; AAP Committee, 2017). Primary healthcare providers should become very familiar with these guidelines.

The AAP does not support the concept of performing the initial newborn hearing screening test in the medical home rather than at the hospital (American Board of Audiology $[A B A], 2016)$. The responsibility of the medical home is to refer infants for further testing if needed. Newborn hearing screening has been successfully implemented over the past two decades, in part because over 95 percent of newborns are delivered in a hospital and have immediate access to a hospital-based program to perform a physiologic test to screen for hearing. The success of these programs is due in part to the captive audience of newborns and has resulted in an efficient, cost-effective implementation with greater standardization of protocols, technology, and accuracy. In addition, the hospital-based institutional commitment to equipment calibration and oversight by qualified audiologists in the hospital setting allows for a quality standard that may be difficult to duplicate when screening is performed in the medical office setting.

The AAP recommends the first newborn hearing screening test be completed at the birthing hospital (AAP, 2014a); however, there may be an occasional situation when this is not possible. Examples include infants born at home and not screened by the midwife or birth attendant, infants whose parents decline hospital-based screening but later realize the merits of screening and consent to office-based screening, and infants who were inadvertently missed at the hospital for any reason. If, on these rare occasions, the first newborn hearing screen is performed in the medical office, all of the guidelines concerning equipment needs, screening techniques, follow-up, and reporting of results to state entities would apply (AAP, 2014a, 2014b).

\section{Improving EHDI Loss-to-Follow-Up/Loss-to- Documentation Rates}

Hearing screeners in the hospital or medical home, and state EHDI coordinators should be aware of some of the following situations under which infants may be lost to the UNHS system (American-Speech-Language-Hearing Association [ASHA], 2008b): 
- Home births and other out-of-hospital births:

$\checkmark$ States should develop a mechanism to

systematically offer newborn hearing screening for all out-of-hospital births.

- Across-state-border births:

$\checkmark$ States should develop written collaborative agreements among neighboring states for sharing hearing-screening results and follow-up information.

- Hospital screenings that are not completed prior to discharge:

$\diamond$ When infants are discharged before the hearing screening is performed, a mechanism should be in place for the hospital to contact the family and arrange for an outpatient hearing screening.

- Transfers to in-state or out-of-state hospitals:

$\diamond$ Discharge and transfer forms should contain the information regarding whether a hearing screening was performed and the results of any screening.

$\diamond$ The recipient hospital should complete a hearing screening if one was not previously performed, or if there is development of a new risk factor for being deaf or hard of hearing.

A pathway for initial screening for infants who, for a variety of reasons, miss the initial screening in the birth hospital should be developed and followed such that no infant will be inadvertently missed, either in the birth hospital, subsequent hospital(s), or after a home-birth. See the National Institute for Children's Health Quality website (NICHQ, 2016) for more information and suggested pathways.

\section{Pediatric Diagnostic Audiology}

Audiologic diagnosis of the infant is the sole purview of the audiologist with specific skills, knowledge, and access to all necessary equipment for infant and early childhood audiologic diagnostic evaluations. It is incumbent upon the audiologist who lacks experience or equipment to refer infants to audiology centers where timely and comprehensive evaluation can be accomplished. Only through consultation with such an audiologist can accurate diagnosis occur, and timely early intervention for the infant and family be assured. Pediatric audiologists and facilities can be discovered through the roster of Pediatric Board Certified audiologists on the ABA website

(http://www.boardofaudiology.org) and the Early Hearing Detection and Intervention-Pediatric Audiology Links to Service (EHDI PALS) website (http://www.ehdi-pals.org). For more information about the searchable EHDI PALS facility directory, see Chung and colleagues (2017).

\section{Key Components of an Audiologic Diagnostic Evaluation in an Infant}

Audiologic diagnosis in the infant must be conducted in a timely manner by audiologists skilled in infant assessment with access to all necessary equipment using evidence-based protocols. Testing is performed to quantify frequency-specific thresholds for air- and bone-conduction stimuli, and to determine the type and degree of hearing loss in each ear to guide the fitting of hearing aids.
In keeping with the 1-3-6 (or 1-2-3) EHDI goals, audiologic diagnosis should be completed no later than 2-3 months of age. This earlier age facilitates the diagnostic process as infants are more likely to sleep for prolonged periods of time required to complete all measures. In children with special health needs, delay in diagnosis of hearing loss may be unavoidable due to attention paid to other health/ time-urgent diagnostic and treatment procedures; however, every effort should be made to minimize the delays. When possible, audiologists can evaluate infants in the NICU, pediatric intensive care unit, or in conjunction with examinations or procedures conducted with general anesthesia or sedation.

The key aspects of audiologic assessment for infants and young children are:

- Auditory brainstem response is the gold standard test for threshold estimation for infants and children who cannot complete behavioral audiologic assessment. ABR provides ear- and frequency-specific threshold estimates that are necessary for the diagnosis of the type, degree, and configuration of hearing loss and provision of amplification (Gorga et al., 2006).

- Measures of middle ear function should be completed as part of the diagnostic audiologic process for infants and young children. Either tympanometry or wideband reflectance can be used to characterize middle ear function (Hunter et al., 2013).

- Acoustic reflexes are an important test of middle ear function and the integrity of auditory brainstem path ways (de Lyra-Silva et al., 2015).

- Otoacoustic emissions provide important information about the integrity of the outer hair cells of the cochlea and provide critical information about the differential diagnosis of auditory neuropathy spectrum disorder and sensorineural hearing loss (Gorga et al., 2000).

- Behavioral assessment of hearing is the gold standard for estimation of hearing thresholds. Visual reinforcement audiometry (VRA; for infants 6-24 months; Widen et al., 2005) and condition play audiometry (CPA; for toddlers 24+ months; Norrix, 2015) are established methods based on conditioned responses to sound.

The following section provides additional detail about the research regarding diagnostic audiological assessment for infants and young children.

Auditory brainstem response evoked potentials using click and frequency-specific stimuli. Auditory brainstem response for estimation of hearing thresholds is the gold-standard for determination of hearing thresholds under 6 months of age. Because ABR is not a test of hearing but rather a measure of an electrophysiologic response to auditory stimulation, confirmation of hearing (perception) requires behavioral evaluation as soon as the child is developmentally capable of providing reliable and valid behavioral responses to sound. It is theoretically 
possible that an infant can have normal ABR recordings, yet not be able to perceive or understand the signal, since comprehension occurs at a higher level in the brain than the sites from which an ABR is recorded.

In the diagnostic $A B R$, recording of electrophysiologic response requires that the newborn or infant sleep soundly for a prolonged period of time so that quiet responses, unmarred by artifact and noise, can be obtained. In some cases, sedation or anesthesia is required to ensure sufficient quiet time for all diagnostic measures to be completed; however, in the young infant with appropriate preparation, natural sleep recordings are quite feasible. Frequency-specific (toneburst) stimuli are used to elicit neural responses that enable determination of thresholds, and form the foundation for determining hearing aid amplification characteristics. Thresholds for both air-conducted and bone-conducted stimuli are measured to determine type (i.e., conductive, sensorineural, mixed) of hearing loss. Bone conduction thresholds are necessary to estimate additional hearing aid gain and output if there is a conductive component. When the ABR shows no response, a specialized protocol (high-intensity click stimulus at positive and negative polarities) should be completed to assess possible auditory neuropathy. The hallmark of the auditory neuropathy ABR is a prominent cochlear microphonic that follows the stimulus polarity when it is reversed. Waveforms subsequent to the polarity-reversing cochlear microphonic are typically absent or significantly aberrant (e.g., poorly-defined, delayed and/or low-amplitude subsequent waveforms). This ABR protocol is the only-recognized and substantiated method for determining auditory neuropathy (Starr, Picton, Sininger, Hood, \& Berlin, 1996).

Research has indicated a good correlation between ABR toneburst thresholds and behavioral thresholds in the middle to high frequencies in infants and young children (Gorga et al., 2006; McCreery, Kaminski, et al., 2015). For infants and young children displaying sensorineural hearing loss, toneburst $A B R$ hearing threshold estimates range from $5 \mathrm{~dB}$ better to $5 \mathrm{~dB}$ poorer than the nominal intensity, depending on the stimulus presentation intensity and frequency. A single correction factor for predicting hearing threshold from $A B R$ threshold estimates is less accurate than use of a level-dependent correction factor (McCreery, Kaminski, et al., 2015). For example, ABR results at lower intensities tend to over-estimate hearing thresholds (suggest hearing loss when there is none), while ABR threshold estimates at higher intensities tend to under-estimate the hearing thresholds (suggest hearing is better than it actually is). In keeping with the cross-check principle, while toneburst ABR serves as the basis for the initial hearing aid fitting, it should be accompanied by ear-specific and frequency-specific behavioral response hearing testing using a VRA protocol (conditioned response) beginning at approximately four to five months of age, depending on the infant's developmental status (Widen et al., 2005).

When VRA is conducted according to careful stimulus, response, and conditioning paradigms, valid and reliable thresholds can be obtained from the typically developing infant. Despite the correlation in average ABR threshold responses to behavioral hearing thresholds across all infants, individual differences vary, and for this reason, validation of $A B R$ thresholds by behavioral testing should occur at the earliest opportunity. Further, since it is not uncommon that children exhibit progressive hearing loss in the first months and years of life, on-going audiological evaluation is essential for any child who is at risk for hearing loss, or any child who wears hearing aids.

Although toneburst ABR is the gold-standard for estimating hearing thresholds in the infant, other evoked-response protocols, stimuli, and technologies are emerging that demonstrate frequency specificity, as well as equivalent, if not superior test efficiency (e.g., ABR or Automated Steady-State Response [ASSR] using puretone or broadband [chirp or click-evoked-chirp stimuli], Cebulla \& Elberling, 2015; Cebulla, Lurz, \& Shehata-Dieler, 2014). Any technology, protocol or stimulus used for objective determination of frequency-specific hearing thresholds should be rigorously and independently validated for the ability to accurately predict behavioral hearing thresholds in infants and young children of all ages and all types and degrees of hearing loss.

Use of novel stimuli (brief-tone chirp or click evoked [CE]-chirp) has recently received attention as a potential alternative to click and toneburst stimuli, with reported improvements in frequency-specificity and shortened test duration. Recordings can be made of multiple frequencies simultaneously, and stimuli can be presented binaurally. Binaural presentation, however, decreases the amplitude of the response, and may not be indicated for use in a child with bilateral hearing loss (Cebulla et al., 2014; Ferm, Lightfoot, \& Stevens, 2013). In a study comparing ABR response amplitudes for tone-pip stimuli at four frequencies $(500,1000,2000,4000 \mathrm{~Hz})$ to narrowband CE-Chirps at corresponding frequencies, authors reported increased amplitude with fewer stimulus presentations in infants with normal hearing (Cebulla et al., 2014; Ferm et al., 2013; Stuart \& Cobb, 2014). Use of a correction factor was proposed to increase the accuracy of estimating hearing thresholds.

Van Maanen and Stapells (2010) observed that there are few studies of deaf and hard of hearing infants and young children comparing ASSR thresholds to ABR thresholds. Results of their study demonstrated that hearing thresholds in children could be reliably classified as normal or elevated based on ASSR thresholds. It was noted, however, that there are insufficient data comparing deaf and hard of hearing infants with typically hearing infants and young children using both air- and bone-conduction stimuli. Higher thresholds are seen in preterm infants as compared with full-term infants. This gap resolved by age 18 months, suggesting auditory maturation of preterm infants (Sousa, Didoné, \& Sleifer, 2016). The ability of ASSR to distinguish between normal hearing and mild 
hearing thresholds is limited (Sousa et al., 2016). There is greater variability in ASSR threshold estimates in infants with typical hearing, such that a standard correction factor cannot be generated (Alaerts, Luts, Van Dun, Desloovere, \& Wouters, 2010). Additionally, the mean air-bone gap for low-frequency ASSR thresholds is significantly greater than that for behavioral (visual reinforcement audiometry), with wide variations across infants (Casey \& Small, 2014). Accurate estimates of bone-conduction thresholds as elicited by ASSR have not been reported (Casey \& Small, 2014). As studies are published that examine these relationships over a variety of ages, hearing thresholds, and etiologies, future endorsement may be possible.

Limited data are currently available regarding the relationship between behavioral hearing thresholds and chirp-elicited responses in infants with a variety of types and degrees of hearing loss, warranting further study. Given the substantial literature that supports the sensitivity and specificity of toneburst $A B R$ in estimating type and degree of hearing loss in infants, alternative test protocols must demonstrate distinct advantages to be considered viable alternatives. Such advantages would need to include improved accuracy in auditory threshold estimation across all ages, types and degrees of hearing loss, and reduced duration of testing time.

Emerging data suggest that new approaches to ABR recording, such as using specialized filtering, advanced signal processing techniques, and placing the pre-amplifier at the position of the electrode, may improve the signal-to-noise ratio in children who are not soundly sleeping. Limited data exist demonstrating the validity of frequency-specific hearing levels obtained through the use of these techniques in non-sedated/awake recordings (Cone \& Norrix, 2015). However, independent evidence is insufficient at this time for the $\mathrm{JClH}$ to endorse this methodology for acquisition of reliable and valid $A B R$ or ASSR threshold estimates in a child of any age who is moving, vocalizing, or otherwise not relatively quiet and still.

\section{Middle ear measures. Tympanometry, wideband} reflectance. In the diagnostic audiologic evaluation, measures of middle ear movement assist in the differentiation of conductive and sensory or neural sites. At the time of newborn hearing screening, neonates may have retained amniotic fluid in the middle ear space, resulting in a not-pass outcome. The standard measure for detecting middle ear fluid has long been high frequency tympanometry, due to superior sensitivity and specificity in detecting middle ear fluid or effusion in infants as compared with standard $226 \mathrm{~Hz}$ tympanometry. Use of the 1000 probe tone is recommended up to age 9 months (Hoffmann et al., 2013).

Increasingly, wideband reflectance, rather than tympanometry is being studied and used with neonates, due to reported superior sensitivity and specificity. Prieve, Vander Werff, Preston, \& Georgantas (2013) noted that conductive hearing loss can be detected in infants using either $1000 \mathrm{~Hz}$ tympanometry or wideband reflectance. Reflectance measures are sensitive to transient middle-ear conditions in infants who did not pass birth screening and subsequently passed screening at age 1 month (Voss, Herrmann, Horton, Amadei, \& Kujawa, 2016).

Wideband reflectance is sensitive to middle-ear disorders including otitis media with effusion in infants and children, and has shown high test-retest reliability demonstrated by high interclass correlations (Hunter, Tubaugh, Jackson, \& Propes, 2008). Keefe and Simmons (2003) analyzed wideband reflectance obtained from a two-stage newborn hearing-screening protocol (OAE/ABR), which resulted in a $5 \%$ false-positive rate. Wideband reflectance measures demonstrated that $80 \%$ of the OAE screening referrals had abnormal responses, indicating evidence of

middle-ear dysfunction. Another study of OAE screening in infants showed significantly higher reflectance between 0.63 and $2 \mathrm{kHz}$ in those infants who failed, compared with those who passed OAE screening (Vander Werff, Prieve, $\&$ Georgantas, 2007). The increasing use of wideband reflectance rather than tympanometry to detect middle ear effusion, suggests that this technique may supplement, if not supplant, tympanometry in the coming years (Hunter et al., 2008).

Acoustic reflex thresholds. Measurement of acoustic reflex thresholds is completed using a $1000 \mathrm{~Hz}$ probe-tone for newborns and infants under 9 months of age (de LyraSilva, Sanches, Neve-Lobo, Ibidi, \& Carvallo, 2015). The acoustic reflex can be reliably measured in infants with normal tympanograms, and can assist in the diagnosis of peripheral and neural hearing involvement (de Lyra-Silva et al., 2015). Good reliability has been shown for tonal and broadband stimuli (Kei, 2012), with published normative data. It is important to recognize that in the infant, the intensity of the stimulus tone or noise will be greater than the dial setting, as the infant ear canal is considerably smaller than the standard coupler used for calibration. Normative data for acoustic stapedial reflexes in healthy neonates demonstrated that mean reflexes occurred at 57 $\mathrm{dB} H \mathrm{HL}$ for broadband noise, and ranged from 65-81 dB HL for tonal stimuli (Kei, 2012). As such, caution must be used in setting the upper limit of stimulus intensity used in eliciting the reflex. The acoustic reflex test is particularly helpful in cases where auditory neuropathy is suspected, as the reflexes are expected to be absent. Berlin and colleagues (2005) found that absent or elevated middle ear muscle reflexes in the presence of normal otoacoustic emissions and confirms auditory neuropathy.

Otoacoustic emissions. OAE (distortion product or transient evoked) testing is essential in the pediatric diagnostic evaluation (Holte et al., 2012; Norton et al., 2000a; Prieve, Schooling, Venediktov, \& Franceschini, 2015). OAEs are measureable sounds that occur when the cochlea is stimulated with a low-intensity click or puretone stimuli. The OAEs are recorded via a probe assembly with a microphone, placed in the external ear canal. Diagnostic 
OAEs provide information about the presence/absence of outer hair cell function, from which hearing level (typical vs. elevated) can be inferred, when the middle ear has been shown to be free of effusion. Although it is possible to have OAEs in the presence of mild sensory hearing loss, the magnitude of the emission diminishes with increasingly elevated thresholds, and the emissions are not observed in hearing thresholds greater than 30-35 dB HL. Mild degrees of hearing loss are difficult to define using OAE technology; however, DPOAEs accurately separate normal hearing ears from those with moderate and greater degrees of hearing loss (Gorga et al., 2000). The magnitude (intensity or amplitude) of an infant OAE is greater than in adults, and as such, the detection of the emission is facilitated. Generally, infants with present DPOAEs are predicted to have hearing thresholds better than $30 \mathrm{~dB} \mathrm{HL}$. Infants with absent DPOAEs (in the presence of normal tympanometry or wide band reflectance) are predicted to have hearing thresholds poorer than $30 \mathrm{~dB} \mathrm{HL}$ (American Academy of Audiology [AAA], 2011, 2012). The OAE assessment is not sufficient for determining hearing thresholds, and cannot be used in isolation to determine hearing aid specifications. It is important to remember that the otoacoustic emission only reflects activity in the cochlea. Infants with auditory neuropathy or more central auditory pathologies are expected to have a normal OAE, yet clearly do not have normal auditory function.

\section{Assessing infants and young children who cannot be evaluated using behavioral testing. Approximately} $40 \%$ of young children who are deaf or hard of hearing have coexisting conditions that, in some cases, may render audiologic evaluation challenging (e.g., physical, intellectual, psychological, or emotional needs or barriers; Gallaudet Research Institute, 2013). In the hands of the pediatric audiologist, many of these children can be conditioned to provide valuable behavioral indications of hearing levels. However, some children may never become candidates for conventional/behavioral testing methods for the purpose of determining and validating frequency-specific hearing thresholds. After determination by a pediatric audiologist that behavioral testing cannot yield meaningful audiometric information, electrophysiological testing with sedation or anesthesia may be indicated, if there are no medical contraindications and if the results of the evaluation will influence the treatment or management of the child. Periodic monitoring of hearing status to assess possible delayedonset or progressive hearing loss (e.g., congenital Cytomegalovirus [CMV]) may also require continued use of electrophysiological measurements. This creates a potential dilemma when anesthesia is required for initial and/or repeated ABR measurement for the purpose of diagnosis and monitoring. Recent research has shown the potential risk to cognitive function in the young child who undergoes general anesthesia (Sun et al., 2016). The benefits and risks must be carefully weighed and the $\mathrm{JCIH}$ recognizes that medically fragile children may not be candidates for anesthesia.

If the child is unable to be evaluated using conventional behavioral tests and ABR evaluation using anesthesia is required, it is important to determine whether the outcome of the ABR evaluation will impact treatment decisions (e.g., hearing aid fitting, cochlear implantation, communication approaches). If the results of the ABR evaluation under anesthesia are not expected to change the course of treatment, it is reasonable to question the necessity for undergoing a costly and time-consuming procedure that carries with it a measure of risk. Professional judgment as well as input and observations from the family are necessary to determine if use of amplification and/or other assistive technology has a probable chance of enhancing access to auditory information. Communication is inherent in the decision to provide amplification and to monitor its function, use, and benefit. Successfully maximizing auditory access to sound requires diligence in communication with the family and other professionals involved in the child's intervention plan. Close monitoring provides information about whether hearing sensitivity is stable, fluctuating, or decreasing.

Assessing infants/toddlers with middle ear effusion or retained amniotic fluid. It is not uncommon for middle ear effusion or retained amniotic fluid in the middle ear to persist in infants regardless of hearing status, causing referral from newborn hearing screening and/or a (temporary) conductive hearing loss. Management of middle-ear fluid in the infant should be coordinated by the infant's pediatrician/primary-care provider and/or a pediatric otologist, with the audiologist's input, and in conjunction with the family's preferences. In some cases, myringotomy with or without a pressure equalization tube placement will be necessary to complete the diagnostic evaluation in a timely fashion during the critical developmental period of early infancy (Rosenfeld et al., 2013). Middle ear effusion often complicates and delays the diagnostic process, resulting in delayed diagnosis of hearing loss.

There is increasing evidence that the infant with retained amniotic fluid and/or persistent middle ear fluid is at increased risk for on-going middle ear involvement (Doyle, Kong, Strobel, Dallaire, \& Ray, 2004). It is important, however, to recognize that not all conductive hearing loss is attributable to middle ear fluid. Congenital ossicular abnormalities can be present and can be distinguished by the audiologist using a combination of air- vs. boneconduction threshold estimation, in addition to tympanometry, acoustic reflex threshold tests, and OAE testing; as well as the otologist's examination and radiographic studies (Boone, Bower \& Martin, 2005).

In 2013, the $\mathrm{JCIH}$ recommended that children at-risk for delayed speech and language development due to chronic middle ear conditions receive intervention services. When present from the time of birth, if a conductive hearing loss (or the conductive component of mixed hearing loss) cannot be medically remediated by six months of age, the child should be considered for hearing aid amplification, communication supports, and referral to early intervention services, even if these services may be short-term. Such 
interventions are necessary to address and prevent developmental language delays. This type of situation commonly occurs when providing care for infants with cleft palate or Down Syndrome.

Assessing the infant/toddler with auditory neuropathy. Auditory neuropathy may occur in association with specific conditions (i.e., extreme prematurity, hyperbilirubinemia, hydrocephalus), or it may present in infants where no obvious causative factor is identified (Kim, Choi, Han, \& Choi, 2016). Audiological characteristics include the presence of OAEs and/or cochlear microphonics accompanied by poor morphology of the ABR and absent or elevated middle ear muscle reflexes (Hood, 2015). Puretone audiometry, when the infant is developmentally able to participate in behavioral testing, is typically characterized by bilateral or unilateral fluctuating or stable thresholds which may range from mild to profound (Attias \& Raveh, 2007; AAA, 2013). Some of these children may have severe neurological involvement and may not ever be able to participate in behavioral evaluation (Uhler, Heringer, Thompson, \& Yoshinaga-Itano, 2012).

Until such time that reliable behavioral thresholds can be determined and hearing status appears to be stable, infants with auditory neuropathy may require frequent audiological monitoring to assess progression, improvement, or fluctuation in electrophysiological threshold estimates. ABR monitoring, however, cannot be used to determine or to demonstrate change in hearing sensitivity, as the hallmark Wave $V$ is not present in ABR recordings of infants or children with auditory neuropathy.

Reversal or resolution of auditory neuropathy can occur for diverse reasons including resolving hyperbilirubinemia or decreased intra-cranial pressure (Attias \& Raveh, 2007). Hearing aid amplification should be deferred in children with auditory neuropathy until minimum responses or behavioral hearing thresholds can be established (AAA, 2013). Although Wave $V$ of the $A B R$ is known to correlate with hearing thresholds in cases of conductive or sensorineural hearing loss, the absence of this evoked response in infants with auditory neuropathy poses a challenge in programming hearing aid gain and output relative to a validated hearing aid prescriptive protocol (e.g., Desired Sensation Level; Seewald, Moodie, Scollie, \& Bagatto, 2005). This practice can result in significant over-or under-fitting of hearing aid amplification for infants with auditory neuropathy. When the child is functioning at a developmental age in which reliable behavioral thresholds can be determined, appropriate hearing aid settings can be established and hearing aids fitted.

Cortical Auditory Evoked Potentials (CAEP) are increasingly being investigated to measure detection and discrimination of sound (Cardon \& Sharma, 2013; Cone \& Whitaker, 2013; Sharma, Cardon, Henion, \& Roland, 2011). For the infant with auditory neuropathy where there is no $A B R$ response to determine auditory threshold, the CAEP can be measured to demonstrate auditory awareness at the cortical level. Auditory awareness and discrimination of tonal and speech stimuli can be measured by scalp-recordings of primary auditory cortical responses. CAEPs are now beginning to be used as biomarkers of acoustic threshold and speech perception capacity in children with auditory neuropathy (Campbell, Cardon, \& Sharma, 2011). Recording of CAEPs can yield frequency-specific threshold information for about $50 \%$ of children with auditory neuropathy (Cone \& Whitaker, 2013; He et al., 2013). Although ABR thresholds closely correlate with hearing thresholds, there can be variability in the accuracy of the toneburst ABR threshold estimate in predicting true behavioral hearing thresholds of individual children at specific frequencies (particularly low frequencies). For some children, toneburst $A B R$ threshold estimates will be almost identical to later behavioral thresholds while other children may have differences that would result in over- or underamplification at specific frequencies. For this reason, validation of $A B R$ thresholds by behavioral testing should occur at the earliest opportunity.

\section{Assessing the infant/toddler using behavioral} audiometry. Although physiologic and electrophysiologic measures are the most reliable and valid estimates of hearing in the neonate and young infant, it is only through evaluating behavioral responses to sound that a true measure of hearing is made. It should be noted that Behavioral Observation (sometimes termed BOA or Behavioral Observation Audiometry) is not a conditioned response, and as such, does not yield reliable and valid hearing thresholds on which to base hearing aid selection characteristics. BOA plays a role in the cross-check principle, in that a child who reliably startles to loud sound is unlikely to have profound bilateral hearing loss. A child who does not startle to loud sound, however, cannot be assumed to have a peripheral hearing loss. Because BOA is not a conditioned response, but rather an observation of behavior that follows exposure to sound, it cannot be used to make diagnostic statements about hearing sensitivity (AAA, 2012).

Puretone thresholds are recognized as the gold standard for determining hearing status (Prieve et al., 2013). VRA is a conditioned response and uses the development of auditory localization in the horizontal plane to observe and reinforce head-turn behavior in response to puretone and speech stimuli. When VRA is conducted according to careful stimulus, response, and conditioning protocols, valid and reliable results can be obtained from the typically developing infant (Widen \& Keener, 2003). Audiometric threshold estimates obtained via VRA are referred to as Minimum Response Levels as they reflect the lowest intensity level at which a response is observed (Widen \& Keener, 2003).

For the slightly older child (24+ months, assuming typical development), conditioned play audiometry provides reliable and valid thresholds to speech stimuli, as well as air- and bone-conducted puretone stimuli. Further, word 
and speech perception are tested to provide information about how the child recognizes and discriminates speech stimuli. It is only through behavioral measures that a true picture of hearing can be obtained. Although physiologic and electrophysiologic measures that underlie hearing provide invaluable information in the child who is too young to be tested through behavioral means, perception and understanding of acoustic stimuli can only be measured using behavioral tools (Widen \& Keener, 2003). Infant audiologic assessment and determination of hearing thresholds require not only adherence to best-practices, but also sufficient time, space, skill, appropriate equipment and protocols, and patience.

\section{Referrals}

If the referral for the pediatric diagnostic audiology evaluation did not originate with the infant's primary care provider (PCP), a copy of the diagnostic audiology report should be sent to the PCP with recommendations for medical and otologic evaluations (AAP Committee, 2017; AAP, 2014a, 2014b) and the state EHDI program CDC, 2016a). In addition, a referral to the state Part $C$ early intervention program must be made upon confirmation of a child being deaf or hard of hearing. Although the Part C revised guidelines state the referral must be made within seven days, immediate referral with a goal of 48 hours is recommended by $\mathrm{JCIH}$ in the early intervention best practices document $(\mathrm{JCIH}, 2013)$. Based on the 1-3-6 guidelines, referral to Part $C$ should always be completed as soon as a child is diagnosed as deaf or hard of hearing, and always prior to six months of age. Diagnosis does not imply that thresholds are determined for all test frequencies, but rather, based on key frequencies (e.g., $500 \mathrm{~Hz}$ and $2000 \mathrm{~Hz}$ ), it can be shown through air- and bone-conduction testing that probable permanent threshold elevation exists in one or both ears.

\section{Medical Evaluation}

Completion of a comprehensive medical evaluation is imperative, concurrent with or shortly following confirmation and/or diagnosis of hearing thresholds (mild, moderate, severe, or profound levels) outside the typical range. The purpose of the medical evaluation for children confirmed as deaf or hard of hearing is to attempt to determine the etiology, to identify related conditions, to provide medical/surgical recommendations and treatments, to provide referrals for indicated ancillary services, and to collaborate with the family in informed decision making for their child (Prosser, Cohen, \& Greinwald, 2015). Essential components of the comprehensive medical evaluation are a detailed history comprising hearing screening details including type (OAE or $A A B R$ ), timing (age of infant at time of screen or diagnostic test), and number of screens completed; family perspectives on auditory awareness and responses of their infant; pregnancy and obstetrical history; and a family history, especially as it relates to family members who have been deaf or hard of hearing. Some targeted questions to identify a variety of syndromes associated with children who are deaf or hard of hearing can also be useful. Copies of the diagnostic pediatric audiology test results, both objective and behavioral, should be requested, including whether these objective measures were obtained with or without sedation.

A physical examination should also be performed, and radiologic and laboratory studies should be considered, consistent with best practices as shown in the 2010 American Academy of Pediatrics Early Hearing Detection and Intervention Guidelines for Medical Home Providers (see https://tinyurl.com/y5zzowco). In addition, every infant confirmed as deaf or hard of hearing, with or without middle ear dysfunction, should be referred by the medical home for specialty evaluations including otologic evaluation, genetics evaluation, ophthalmologic evaluation, and additional medical evaluations as indicated (AAP, 2010; Prosser et al., 2015). However, these referrals should not delay the fitting of amplification, which should occur as soon as sufficient audiological information is obtained. It is important that medical professionals be sensitive to the attitude, mood, and perspective of the family when caring for the child who is newly identified or diagnosed as deaf or hard of hearing. The health professional should be prepared to refer the family for counseling and participation in family support groups, in addition to necessary medical, audiological, and earlyintervention services, with the goal that the infant and the infant's family receive optimal family-centered care (Arnold et al., 2006; Davis et al., 2006).

\section{Role of the Pediatrician/Primary Care Provider}

The pediatrician or other primary care provider is responsible for monitoring the general health, development, and well-being of the infant. The PCP plays an important role in monitoring birth hearing screening outcomes, and ensuring follow-up with rescreening and audiologic diagnostic evaluation when indicated. The PCP is part of the team that ensures that the audiologic and medical assessment is conducted in a timely fashion for infants who do not pass screening. Rescreening guidelines are available on the American Academy of Pediatrics EHDI website in addition to other resources (AAP, 2010; AAP, 2014a, 2014b; AAP Committee, 2017). For all infants suspected or confirmed to be deaf or hard of hearing, the PCP must partner with other specialists, particularly the otolaryngologist, audiologist, geneticist/ genetics counselor, and early intervention specialist to facilitate coordinated and comprehensive care for the infant and family. In 2010, AAP developed the medical-home algorithm (guidelines) for management of infants suspected or confirmed as deaf or hard of hearing (see https://tinyurl.com/y5zzowco).

Middle-ear status should be monitored by the PCP, because the presence of middle-ear effusion has the potential to delay diagnosis of hearing and can further compromise hearing. Surveillance of both middle ear status and developmental milestones is recommended, regardless of the infant's birth hearing screening results or 
hearing threshold levels (Rosenfeld et al., 2013). Prompt specialty referrals should follow when new or delayed-onset conditions are suspected (Gracey, 2003). Because approximately forty percent of children confirmed as deaf or hard of hearing will demonstrate additional conditions or delays such as autism, blindness, learning differences, genetic syndromes, et cetera, health care providers have an important role in confirming that these children are receiving comprehensive services (Roizen et al., 2014). Regardless of the newborn hearing screening results, the pediatrician or PCP should review every infant'smedical and family history for the presence of known risk indicators that require monitoring for delayedonset or progressive hearing loss (see Table 1). The PCP should also ensure that an audiologic evaluation is completed for these children as recommended (Coenraad, Goedegebure, van Goudoever, \& Hoeve, 2010; Fligor, Neault, Mullen, Feldman, \& Jones, 2005; Fowler, 2013; Nance, Lim, \& Dodson, 2006). In addition, the PCP is responsible for ongoing surveillance of family concerns about speech, language, hearing, auditory skills, and developmental milestones of all infants and children regardless of risk status, as outlined in the pediatric periodicity schedule published by the American Academy of Pediatrics (AAP Committee, 2017).

A growing body of research indicates that children who receive cochlear implants $(\mathrm{Cl})$ are at increased risk for developing bacterial meningitis over the general population (Biernath et al., 2006; Gluth, Singh, \& Atlas, 2011; Melton \& Backous, 2011; Parner et al., 2007). Historically, cochlear implant devices that specifically involved a separate electrode positioner appeared to confer a much higher risk of meningitis over other devices, but have since been eliminated from the market. Starting in 2002, the CDC established guidelines for additional immunizationagainst bacterial meningitis in children with cochlear implants, to be implemented in addition to already-established routine prophylactic vaccinations recommended for all children. The current recommendations of the CDC and FDA vaccine programs are available on the CDC website (CDC, 2016b).

\section{Role of the Otolaryngologist}

Otolaryngologists are physicians and surgeons who diagnose, treat, and manage a wide range of diseases of the head and neck, including ear, hearing, and vestibular disorders. They perform a full medical diagnostic evaluation of the head and neck, ears, and related structures, including a comprehensive history and physical examination, leading to a medical diagnosis and appropriate medical and surgical management (Rutherford, Lerer, Schoem, \& Valdez, 2011). The otolaryngologist's evaluation includes a comprehensive history to identify the presence of risk factors for congenital or delayed-onset childhood hearing loss (see Table 1; Morzaria, Westerberg, \& Kozak, 2005), and a physical examination that includes examination of the head and neck structures for defects of the auricles (microtia); patency of the external ear canals (atresia); status of the tympanic membranes and middle ear structures; presence of preauricular pits or cysts; positioning of eyes and orbits, cheek bones, and jaws; and evidence of facial asymmetry. Additional findings associated with congenital deafness and elevated hearing thresholds include focal hair discoloration with wide-appearing eyes (dystopia canthorum), or different colored eyes. An evaluation for an enlarged thyroid gland should also be performed.

Relatively common syndromes associated with observable physical anomalies and permanent sensorineural hearing loss include: Waardenburg syndrome, CHARGE syndrome, VACTERL syndrome, branchio-oto-renal syndrome, and Pendred syndrome (Martini, Calzolari, \& Sensi, 2009). Usher syndrome is associated with progressive blindness secondary to retinitis pigmentosa, a degenerative eye disease, and is a common cause of deaf-blindness (Kimberling et al., 2010).

Congenital permanent hearing loss or transient/fluctuating conductive hearing thresholds may be associated with craniofacial anomalies such as cleft lip and/or cleft palate (Nicholson, Christensen, Dornhoffer, Martin, \& SmithOlinde, 2011). Children with craniofacial abnormalities, despite the absence of middle ear fluid, may have permanent conductive hearing threshold changes secondary to abnormalities of the ossicular chain or Eustachian tube anatomy/physiology. Tympanic membrane visualization may reveal middle ear abnormalities such as a congenital ossicular deformity, middle ear effusion, or cholesteatoma. Syndromes associated with conductive hearing loss might include: Down syndrome, TreacherCollins syndrome, Crouzon syndrome, Klippel-Feil syndrome, branchio-oto-renal syndrome, CHARGE syndrome, VACTERL syndrome, and Goldenhar syndrome (Kimberling et al., 2010; Nicholson et al., 2011; Acke, Dhooge, Malfait, \& De Leenheer, 2012; Blanco-Kelly et al., 2015; Lammens, Verhaert, \& Desloovere, 2013; Rajenderkumar, Bamiou, \& Sirimanna, 2005).

The otolaryngologist will also assess for transient conductive hearing changes secondary to amniotic fluid/ debris in the ear canal, cerumen occluding the ear canal, or middle ear fluid. Whereas middle ear fluid may constitute a transient condition in the infant with typical anatomy, the possibility of long-standing, fluctuating chronic middle ear fluid in children with cleft palate, Down syndrome, or other craniofacial anomalies must be considered in terms of requiring amplification and/ or visual communication due to prolonged periods of time with compromised audibility of speech sounds (Nicholson et al., 2011; McCreery, Walker, et al., 2015).

Radiologic imaging. Temporal bone imaging is indicated when families seek the etiology for children who are suspected to be deaf or hard of hearing, as it may further characterize the etiology, identify anatomical markers for progression, predict prognosis from interventions such as amplification and/or cochlear implantation, and identify lesions of the central nervous system that require medical/surgical intervention for the 
Table 1

Risk Factors for Early Childhood Hearing Loss: Guidelines for Infants who Pass the Newborn Hearing Screen

\begin{tabular}{|c|c|c|c|}
\hline & Risk Factor Classification & $\begin{array}{l}\text { Recommended } \\
\text { Diagnostic Follow-up }\end{array}$ & Monitoring Frequency \\
\hline & Perinatal & & \\
\hline 1 & $\begin{array}{l}\text { Family history* of early, progressive, or delayed onset permanent } \\
\text { childhood hearing loss }\end{array}$ & by 9 months & $\begin{array}{l}\text { Based on etiology of family hearing loss and } \\
\text { caregiver concern }\end{array}$ \\
\hline 2 & Neonatal intensive care of more than 5 days & by 9 months & \multirow{4}{*}{$\begin{array}{l}\text { As per concerns of on-going surveillance of } \\
\text { hearing skills and speech milestones }\end{array}$} \\
\hline 3 & $\begin{array}{l}\text { Hyperbilirubinemia with exchange transfusion regardless of length } \\
\text { of stay }\end{array}$ & by 9 months & \\
\hline 4 & Aminoglycoside administration for more than 5 days ${ }^{* *}$ & by 9 months & \\
\hline 5 & Asphyxia or Hypoxic Ischemic Encephalopathy & by 9 months & \\
\hline 6 & Extracorporeal membrane oxygenation (ECMO) ${ }^{\star}$ & $\begin{array}{l}\text { No later than } 3 \text { months } \\
\text { after occurrence }\end{array}$ & $\begin{array}{l}\text { Every } 12 \text { months to school age or at shorter } \\
\text { intervals based on concerns of parent or provider }\end{array}$ \\
\hline \multirow[t]{4}{*}{7} & $\begin{array}{l}\text { In utero infections, such as herpes, rubella, syphilis, and } \\
\text { toxoplasmosis }\end{array}$ & by 9 months & As per concerns of on-going surveillance \\
\hline & In utero infection with cytomegalovirus (CMV)* & $\begin{array}{l}\text { No later than } 3 \text { months } \\
\text { after occurrence }\end{array}$ & $\begin{array}{l}\text { Every } 12 \text { months to age } 3 \text { or at shorter intervals } \\
\text { based on parent/provider concerns }\end{array}$ \\
\hline & $\begin{array}{l}\text { Mother + Zika and infant with no laboratory evidence \& no clinical } \\
\text { findings }\end{array}$ & standard & As per AAP (2017) Periodicity schedule \\
\hline & $\begin{array}{l}\text { Mother + Zika and infant with laboratory evidence of Zika + clinical } \\
\text { findings } \\
\text { Mother + Zika and infant with laboratory evidence of Zika - clinical } \\
\text { findings }\end{array}$ & $\begin{array}{l}\text { AABR by } 1 \text { month } \\
\text { AABR by } 1 \text { month }\end{array}$ & $\begin{array}{l}\text { ABR by } 4-6 \text { months or VRA by } 9 \text { months } \\
\text { ABR by 4-6 months } \\
\text { Monitor as per AAP (2017) Periodicity } \\
\text { schedule (Adebanjo et al., 2017) }\end{array}$ \\
\hline 8 & $\begin{array}{l}\text { Certain birth conditions or findings: } \\
\text { - Craniofacial malformations including microtia/atresia, ear } \\
\text { dysplasia, oral facial clefting, white forelock, and microphthalmia } \\
\text { - Congenital microcephaly, congenital or acquired hydrocephalus } \\
\text { - Temporal bone abnormalities }\end{array}$ & by 9 months & $\begin{array}{l}\text { As per concerns of on-going surveillance of } \\
\text { hearing skills and speech milestones }\end{array}$ \\
\hline \multirow[t]{2}{*}{9} & $\begin{array}{l}\text { Over } 400 \text { syndromes have been identified with atypical hearing } \\
\text { thresholds }{ }^{\star \star \star} \text {. For more information, visit the Hereditary Hearing } \\
\text { Loss website (Van Camp \& Smith, 2016) }\end{array}$ & by 9 months & $\begin{array}{l}\text { According to natural history of syndrome or } \\
\text { concerns }\end{array}$ \\
\hline & Perinatal or Postnatal & & \\
\hline 10 & $\begin{array}{l}\text { Culture-positive infections associated with sensorineural hearing } \\
\text { loss }{ }^{* * *} \text {, including confirmed bacterial and viral (especially herpes } \\
\text { viruses and varicella) meningitis or encephalitis }\end{array}$ & $\begin{array}{l}\text { No later than } 3 \text { months } \\
\text { after occurrence }\end{array}$ & $\begin{array}{l}\text { Every } 12 \text { months to school age or at shorter } \\
\text { intervals based on concerns of parent or provider }\end{array}$ \\
\hline 11 & $\begin{array}{l}\text { Events associated with hearing loss: } \\
\text { - Significant head trauma especially basal skull/temporal bone } \\
\text { fractures } \\
\text { - Chemotherapy }\end{array}$ & $\begin{array}{l}\text { No later than } 3 \text { months } \\
\text { after occurrence }\end{array}$ & According to findings and or continued concerns \\
\hline 12 & $\begin{array}{l}\text { Caregiver concern }{ }^{\star * * *} \text { regarding hearing, speech, language, } \\
\text { developmental delay and or developmental regression }\end{array}$ & Immediate referral & According to findings and or continued concerns \\
\hline
\end{tabular}

Note. $\mathrm{AAP}=$ American Academy of Pediatrics; $\mathrm{ABR}=$ auditory brainstem response; $\mathrm{AABR}=$ automated auditory

brainstem response.

* Infants at increased risk of delayed onset or progressive hearing loss

${ }^{* *}$ Infants with toxic levels or with a known genetic susceptibility remain at risk

*** Syndromes (Van Camp \& Smith, 2016)

****Parental/caregiver concern should always prompt further evaluation.

overall health of the patient (Lowe \& Vézina, 2005).

Malformations of the external, middle, and inner ear as

well as the internal auditory canal are clearly detectable using currently available imaging. Structural anomalies of the cochleovestibular nerves and brain are also discernable in most cases. In general, high resolution computed tomography (HRCT) is well-suited for assessing the osseous structures (external auditory canal and middle ear), while magnetic resonance imaging (MRI) provides excellent soft tissue and fluid detail for looking at the cranial nerves and brain (Lowe \& Vézina, 2005). The inner ear including the vestibular aqueduct (endolymphatic duct) is well visualized using either MRI or HRCT. 
For cases of aural atresia and other conductive conditions, HRCT remains superior to MRI for assessing bony detail. For children with newly identified sensorineural or mixed types of hearing loss, MRI allows direct imaging of the inner ear, cochlear nerves, and brain without the need for cranial irradiation. These imaging studies may also be used to assess potential candidacy and prognosis for surgical intervention, including reconstruction, bone conduction hearing devices or implants, and cochlear implantation. For cochlear implantation, MRI is usually sufficient except in select situations where facial nerve anomalies, inner ear ossification (Adunka, Jewells, \& Buchman, 2007), patency of the bony cochlear nerve canal (Adunka et al., 2007), and temporal bone pathology (Roche et al., 2010) are in question. The finding of cochlear nerve deficiency or severe inner ear malformations on imaging may directly affect the prognosis for the development of open-set speech perception and/or spoken language using amplification or cochlear implants (Young, Ryan, \& Young, 2014).

\section{Additional testing considerations. Additional} laboratory testing to be considered in the process of evaluating a newborn or infant diagnosed with sensorineural hearing loss and to rule out the most common etiologies that impact intervention plans include (a) connexin gene, (b) electrocardiogram, and (c) urine and saliva swabs for congenital cytomegalovirus (cCMV). While connexin and electrocardiogram tests are briefly mentioned here, due to their importance they are covered in more depth in the next section regarding genetic testing. Furthermore, significant evidence is presented in the cCMV section to support $\mathrm{JCIH}$ recommendations.

Connexin 26 and Connexin 30 genes. Mutations in this gene account for a significant number of nonsyndromic cases (Orzan \& Muria, 2007; Tarkan et al., 2013). As the inheritance is autosomal recessive, these children frequently have a negative family history for other family members who are deaf or hard of hearing. In the absence of other risk factors, these children may or may not pass the birth screen. Developmental surveillance and parental concern play an important role in monitoring infants who are unknowingly at risk for progressive hearing changes due to this genetic condition.

Electrocardiogram. An electrocardiogram is carried out among children who are profoundly deaf to identify a long QT interval (Jervell and Lange-Nielsen syndrome; Tarkan et al., 2013). This rare autosomal recessive inherited condition can result in sudden death if undetected and therefore, the benefit far outweighs the cost of what may seem an unnecessary medical procedure.

Congenital Cytomegalovirus (cCMV). CMV infection is a leading cause of congenital infection, occurring in 0.2 to 2 percent of live births worldwide and is a leading cause of non-genetic unilateral or bilateral sensorineural hearing loss (Cannon, Griffiths, Aston, \& Rawlinson, 2014; Doutre, Barrett, Greenlee, \& White, 2016; Ross et al., 2014;
Yamamoto et al., 2011). About 25,000 infants are born each year in the United States with congenital CMV infection, 10-15\% of whom develop sensorineural hearing loss (Cannon et al., 2014). Since children with cCMV can develop late onset sensorineural hearing loss (Cannon et al., 2014), all infants who test positive on a neonatal screen for CMV require periodic monitoring by audiology to identify changes in hearing thresholds, with the provision of appropriate amplification and early intervention as indicated.

A standardized, high-throughput test suitable for cCMV newborn screening does not currently exist, but development of an appropriate and relatively inexpensive screening is a priority research topic (NIDCD, 2002). Another research priority is antiviral treatment of newborns that have cCMV. Administration of parenteral ganciclovir has shown protection against hearing deterioration in some studies but not others, and as there are concerns about toxicity, randomized control trials are needed (Hamilton et al., 2014; Smets et al., 2006).

\section{Role of the Geneticist and the Genetics Counselor}

The medical geneticist is responsible for the interpretation of family history data, the clinical evaluation and diagnosis of inherited disorders, and the performance and assessment of genetic tests. Geneticists, along with genetic counselors, provide genetic counseling to families. Geneticists and genetic counselors are uniquely qualified to interpret the significance and limitations of tests and to convey the current status of knowledge during genetic counseling. All families of children who are confirmed as deaf or hard of hearing should be offered a genetics evaluation and counseling (Alford et al., 2014). This evaluation can provide families with information on etiology, prognosis for progression, associated disorders (e.g., renal, vision, cardiac), and likelihood of recurrence in future offspring (or the offspring of close relatives). Occasionally, a genetic determination can identify a particular syndrome that is associated with otherwise unrecognized medical conditions that require intervention.

Incidence of genetic disorders. In large population studies, at least fifty percent of the etiologies related to being deaf or hard of hearing are designated as hereditary; hundreds of syndromes and many different individual genes have been identified (Morton \& Nance, 2006; Santos et al., 2005). The evaluation, therefore, should include a review of family history of specific genetic disorders or syndromes, genetic testing for gene mutations such as GJB2 (connexin-26), and syndromes commonly associated with early-onset hearing loss (see Risk Factors, Table 1). As the widespread use of newly developed conjugate vaccines decreases the prevalence of infectious etiologies such as measles, mumps, rubella, Haemophilus Influenza Type B, and childhood meningitis, the percentage of cases of early onset hearing loss attributable to genetic etiologies can be expected to increase, thereby improving the utility of the recommendation for early genetic evaluations (Alford et al., 
2014). Connexin 26 and 30 genes code for the production of gap junction proteins, which allow for ion chemical balance (potassium) in the inner ear. Mutations in the connexin 26 (and to a lesser degree connexin 30 ) genes account for a significant number of non-syndromic cases. The decision to obtain genetic testing is dependent on informed family choice in conjunction with standard confidentiality guidelines (Chu et al., 2015).

Genetic testing. Although an individual family may choose not to participate in genetic testing for a variety of reasons, the American College of Medical Genetics recommends offering genetic counseling and genetic testing for all infants who are deaf or hard of hearing and their families (Alford et al., 2014). New technologies are changing the genetic diagnostic process. Chromosome microarray technology (looking for known micro-deletions and micro-duplications), with an expected yield of $15-18 \%$ in a child with malformations or developmental delay, is beginning to replace routine karyotyping, since most of these alterations are not visible on routine cytogenetics (Chu et al., 2015). In the near future, multi-gene panels may replace some of the diagnostic studies previously done on non-syndromic patients. For example, molecular testing is available for Usher syndrome, the long QT syndromes and the hereditary nephropathies. The panel also includes testing for Connexin 26 and other common gene abnormalities producing pre-lingual and post-lingual non-syndromic hearing loss.

Counseling about the results of genetic testing may influence the family's decisions regarding intervention options for their child. A recent Australian longitudinal study suggests that genetic testing can be valuable in determining etiology (Dahl et al., 2013). This study completed molecular testing of perinatal Guthrie newborn dried blood spots of children identified as deaf or hard of hearing either as neonates or after the newborn period (Dahl et al., 2013). They analyzed the GJB2 and SLC26A4 genes for the presence of mutations, screened for the mitochondrial DNA A1555G mutation, and screened for congenital CMV infection in DNA. Results were obtained for 364 children and etiology was established for sixty percent of these children. One or two known GJB2 mutations were present in 82 of the children. Twenty-four children had one or two known SLC26A4 mutations. GJB2 or SLC26A4 changes with unknown consequences on hearing were found in 32 children. The A1555G mutation was found in one child, and CMV infection was detected in 28 children. Auditory neuropathy was confirmed in 26 children whose DNA evaluations were negative.

Every identified infant should have a regular evaluation by an ophthalmologist to document visual acuity and rule out concomitant or delayed-onset vision disorders, such as cataracts or Usher syndrome (Dammeyer, 2012). Indicated referrals to other medical subspecialists, including developmental pediatricians, neurologists, cardiologists, and nephrologists, should be facilitated and coordinated by the PCP.
Timely intervention for the infant. Ensuring that the identified child has access to developing receptive and expressive language is of paramount importance. Whether that language is spoken, sign (e.g., American Sign Language) or a combination (bilingual) is of lesser importance than ensuring access to language and resultant linguistic competence (Kushalnagar et al., 2010; Poliva, 2016). The JCIH Supplement on Early Intervention $(\mathrm{JClH}, 2013)$ addresses best-practices in language development and intervention.

The great majority of infants identified through UNHS programs have sufficient residual hearing (levels ranging from mild to severe) to permit benefit from hearing aid amplification (Holte et al., 2012). Accordingly, hearing aid amplification is the typical primary treatment for most identified infants. That being said, it would be very much an oversimplification to consider amplification alone as an adequate intervention. A broader discussion of early intervention guidelines was recently published as a supplemental statement by the JCIH (2013), and that supplemental statement provides additional detail not included in the JCIH (2007) statement.

\section{Role of the Audiologist Following Diagnosis}

It is often the audiologist who provides the first information to the family for an identified child following diagnosis. The audiologist has the responsibility for communicating the information to the family in an empathetic, non-biased, open-ended fashion, and in a language (spoken or signed) that is accessible to the parents, using interpreters/ translators as necessary (Fitzpatrick, Durieux-Smith, Eriks-Brophy, Olds, \& Gaines, 2007; Pizur-Barnekow, Darragh, \& Johnston, 2011). Families retain information at different rates and require different styles of information delivery. Because of the complexity and volume of information, redundancy in the message and delivery of the message through multiple avenues (e.g., written, verbal, website, video presentation, etc.) is important.

Most often, the audiologist is responsible for referral to the early intervention system. In some systems, the audiologist is the primary professional who conveys information about early intervention opportunities; in other sites it is an early intervention provider with specialized knowledge and skills in working with children who are deaf or hard of hearing. Early, careful, and comprehensive education of families and caregivers regarding the nature of language acquisition is invaluable in encouraging families to seek appropriate early intervention services for their child.

When counseling families, information regarding communication modes, methodologies, and technologies should be provided in a comprehensive and non-biased fashion. Families should be offered written materials in an accessible format and language. Information about listening and spoken language, signed language, and combined approaches should be provided. Additionally, 
information about amplification options (hearing aids, cochlear implants, visual and auditory assistive technologies) should be provided as appropriate for the infant's audiologic diagnosis, recognizing the possibility of progression of hearing thresholds to a more severe degree (ASHA, 2008a). Families benefit from contact with other parents who are trained to provide parent-to-parent or family-to-family support, and also benefit from contact with a trained professional who is deaf or hard of hearing (Moeller et al., 2013).

\section{Amplification: Hearing aids, cochlear implants, bone conduction hearing devices, assistive hearing technologies}

Hearing aid amplification. Families should be educated about the need for regular audiologic re-evaluation. It is probable that the first confirmation that a child is deaf or hard of hearing does not include, with optimal reliability and validity, detailed information about hearing status at all frequencies important for the development of speech and language (Bagatto et al., 2016). Audiologic management does not stop at the diagnosis. Regular surveillance of hearing status is critical to ensure that hearing aid amplification is appropriately fitted and programmed. Progression or fluctuation of hearing thresholds can occur, and hearing sensitivity may change or fluctuate with concurrent episodes of middle ear effusion.

An important first step for families who have chosen development of listening and spoken language as a goal is the fitting of appropriate hearing aid amplification. If the family chooses, fitting of hearing aid amplification no later than four months of age (or as soon as there is confirmation that the child is deaf or hard of hearing) is optimal, if not medically contraindicated (e.g., draining ear, local skin or ear canal condition, absent auditory nerves; Bagatto et al., 2016).

Following diagnosis of the hearing loss, audiologists should allow ample time to:

- listen to families and to answer their questions;

- support family decision-making;

- provide additional resources;

- provide information and referrals for family support;

- encourage families to advocate for their needs;

- use clear, simple (lay) language;

- explain the process (e.g., referral to early intervention);

- explain what will happen next (e.g., next appointment);

- explain the hearing aid or cochlear implant process, and

- discuss visual strategies and resources.

Hearing aid selection and fitting in infants must be conducted using evidence-based protocols and algorithms (Bagatto et al., 2016). Consistent with pediatric diagnostic evaluations, the fitting of pediatric amplification should be provided by an audiologist with expertise, skills, and knowledge in pediatric audiology (Bagatto et al., 2016). Selection of hearing aids for infants/toddlers must include features specifically designed for this age group: pediatric-sized earhooks, tamper-proof battery doors, and accessibility for remote-microphone (FM) technology. Further, as progressive hearing loss is not uncommon in young infants, hearing aids with flexible gain and output characteristics are necessary to accommodate potential change (decrement) in hearing in the first few months and years of life (Bagatto et al., 2016). Many major hearing aid manufacturers offer loaner hearing aid programs to pediatric audiologists in an effort to minimize the time between diagnosis and hearing aid fitting.

Some current EHDI databases do not contain information about amplification (Uhler, Thomson, Cyr, Gabbard, \& Yoshinaga-Itano, 2014). Suggestions for improving the standardization and reporting to state EHDI data systems have recently been provided (Alam, 2016). Minimally, information collected by EHDI programs should include: (a) Whether amplification was recommended by the audiologist, and (b) The age amplification was first provided (either through loaner or purchase). If there is confirmation that the child is bilaterally deaf or hard of hearing, bilateral hearing aids should be fitted unless medical or audiological contraindication exists or the family does not elect to proceed.

Although toneburst ABR thresholds will most likely be used for initial hearing aid fitting, as soon as developmentally possible, the audiologist should use behavioral thresholds (responses) in addition to ABR thresholds for verifying hearing aid gain and output targets. Although ABR thresholds are good predictors of behavioral thresholds, established behavioral responses are the best reflection of how the child hears (Alam, 2016). To use validated, frequency-specific targets for gain and output, frequencyspecific thresholds (at a minimum, one low-frequency and one high-frequency), as well as a measured estimate of any conductive component through the use of boneconduction $\mathrm{ABR}$ or behavioral test, is necessary.

An emerging technique for hearing aid fitting and validation is through the use of CAEP (Punch, Van Dun, King, Carter, \& Pearce, 2016). Small, Ishida, and Stapells (2017) reported on cortical response recordings demonstrating binaural lateralization and binaural hearing processes. Although not yet in widespread use in the United States, CAEPs have been shown to confirm audibility of speech sounds at the cortical level and to objectively demonstrate auditory discrimination. CAEP can be recorded at a very young age and has the specific advantage of avoiding use of sedation and anesthesia while providing validation of hearing technology (Punch et al., 2016).

Optimization and objective re-verification of hearing aid fitting should occur with each new earmold fitting, and as puretone threshold information changes. Optimization implies programming hearing aid gain and output relative to an evidence-based, validated pediatric amplification target (i.e., Desired Sensation Level, Bagatto et al., 2016; or National Acoustics Laboratories, Ching et al., 2013). Initial amplification, as well as each new earmold fitting 
should be accompanied by (re-)measuring the child's real ear to coupler difference (RECD) with the earmold in place to optimize the hearing aid settings to individual childspecific amplification targets across the speech spectrum. Regular audiologic evaluation is required to monitor any fluctuation or decrement in hearing; should changes in hearing sensitivity occur, hearing aid programming and options may require modification to accommodate the change in hearing. Ongoing validation of amplification fitting is mandatory to ensure that the purpose of hearing aids (i.e., development of spoken language, auditory awareness, or other) is being fulfilled by the amplification arrangement (Bagatto et al., 2016). An adequate earmold fit is critical, and new earmolds are required as the child grows; this is a particular concern in infants, for whom more rapid ear growth can be anticipated.

The purpose of hearing aid amplification in infants who are deaf or hard of hearing is to facilitate timely and optimal auditory development as a precursor to development of spoken language (Meinzen-Derr, Wiley, Creighton, \& Choo, 2007; Wiley \& Meinzen-Derr, 2013). The expectations for timely progress in acquiring spoken language, of course, must be individualized, based on the nature (type) and degree of hearing loss, the child's other special needs (if any), the early intervention program, and the level of family involvement. Progress in developing effective communication using listening and spoken language must be regularly assessed. If the infant/child is not meeting expected language milestones with appropriately fitted hearing aid amplification alone, additional language and communication approaches andor technologies should be considered. Approaches might include ASL, cochlear implantation, or additional hearingassistance technologies (Wiley \& Meinzen-Derr, 2013).

\section{Assistive technologies. Assistive technologies} encompass a variety of devices, both auditory and visual as well as vibrotactile. Technologies include listening devices beyond the hearing aid, such as wireless transmission from a remote microphone, visual communication technologies such as CART (communication access realtime translation), Skype or computer-based video transmission, devices to amplify telephone communications, and devices to provide visual translation of auditory stimuli in the home such as flashing doorbells. Remote-microphone technology is optimal in situations involving noise and distance. Once a child begins to spend more time at distances away from the person speaking or in noisy situations (e.g., car), the use of hearing assistive technologies as well as visual and tactile assistive technologies should be considered.

\section{Bone conduction hearing device/implant} considerations, requirements, expectations. Continuing audiological and medical surveillance as well as provision of information and education and support for the family is necessary to optimize development and treatment for the infants and toddlers who are deaf or hard of hearing. Medical management varies, ranging from the removal of cerumen from the ear canal, to the treatment of concurrent middle ear effusion or infection, to long-term plans for reconstructive surgery and assessment of candidacy for cochlear implants. If necessary, surgical treatment of malformation of the outer and middle ears (including bone conduction hearing devices) should be considered in the intervention plan for infants and toddlers who are deaf or hard of hearing with permanent conductive or mixed hearing thresholds when a child reaches an appropriate age (Whitton \& Polley, 2011). If surgical treatment is planned, evaluation of hearing needs as well as cosmetic gains must both be considered. Infants and young children can be fit with and wear bone conduction hearing devices on a softband until they are considered potential implant candidates at age five. Surgical treatment that is designed to improve cosmetic appearance of the external ear may impede successful use of hearing aids as a result of changes in the pinna or ear canal.

Cochlear implantation considerations, requirements, expectations. If the parents' goals for their child include development of spoken language, cochlear implants are the mainstay of treatment for most children who are deaf with thresholds in the severe to profound range. Cochlear implants are indicated for children (>12 months of age) with bilateral severe-to-profound sensorineural hearing loss (including auditory neuropathy) who fail to make expected progress with appropriately fitted amplification. Comprehensive pediatric hearing centers integrate the assessment and intervention phases for children who are deaf, making hearing aid and cochlear implant transitions seamless. Minimally, audiologists and PCPs must work together with the parents and early intervention specialists to recognize implant candidates and make prompt referrals.

At the present time, there is no single comprehensive best-practices document regarding pediatric cochlear implantation. Cochlear implant surgery around 12 months of age or younger offers the greatest chance of significant open-set speech understanding with resulting language acquisition rates that match those of normal hearing peers.

Timing of the intervention remains critical, with better outcomes achieved for those receiving an implant by two years of age (Ching et al., 2009; Dettman, Pinder, Briggs, Dowell, \& Leigh, 2007). Studies have documented the critical nature of early implantation for the development of spoken language (Niparko et al., 2010). It is noteworthy that nearly all children with no responses to multifrequency toneburst $\mathrm{ABR}$ are audiologically cochlear implant candidates (Hang et al., 2015). With this in mind, these children should be considered for fast-tracking surgery in the first year of life to avoid unnecessary delays.

Cochlear implants can be provided unilaterally or bilaterally (which may be simultaneous or sequential), and can be used in combination with amplification (i.e., bi-modal/hearing aid in one ear and cochlear implant in the other) [Ching \& Dillon, 2013; Scherf et al., 2009a, 2009b). 
Binaural (i.e., two ear) hearing improves sound localization and hearing in noise. For children, the binaural benefit presumably can enhance incidental learning, an important skill for language development. Although there still remains some controversy regarding the relative benefits of bimodal versus bilateral implantation, it is clear that the binaural benefit is best achieved for children receiving two implants (Steel, Papsin, \& Gordon, 2015). Moreover, bilateral implants offer a built-in backup system to avoid complete loss of sound if one device fails or is lost or broken. Hybrid cochlear implants that combine electrical and acoustic stimulation of the auditory system in the same ear are FDA-approved for use in adults, but have yet to be tested or approved for use in infants or children. It is probable that this emerging and potentially-beneficial technology will become available and approved for children in the future (Gantz et al., 2010).

The decision as to whether a child is a cochlear implant candidate begins with the audiological and medical assessment. For children who are appropriate candidates, the decision to pursue implantation is then the choice of the family, based on their communication desires for their child. The family must also have reasonable expectations and a clear understanding of the continued need for intensive, auditory-based intervention and audiological management (Weisel, Most, \& Michael, 2007).

Outcomes following cochlear implantation can be impacted by a number of variables that include: age at implantation (Ching et al., 2009; Dettman et al., 2007; Niparko et al., 2010), progression of hearing loss (Barreira-Nielsen et al., 2016; Fitzpatrick, Ham, \& Whittingham, 2015), duration of device use, cochlear morphology and cranial nerve VIII integrity as determined on imaging (Buchman et al., 2011), precise electrode placement in the scala tympani, presence of additional neurodevelopmental challenges, high quality mapping of the speech processor, parental educational level, and involvement in family centered, intensive auditory-based intervention (Iseli \& Buchman, 2015).

Referral to early intervention services. The purpose of early intervention is to achieve optimal child and family outcomes. Hence, the audiologist must make the referral for Part C Early Intervention (IDEA, 2004) services as quickly as possible following confirmation that a child is deaf or hard of hearing. Federal regulations require that this referral be made within seven working days of diagnosis (IDEA, 2004). In cases of congenital aural atresia, the referral can and should be made by the birth hospital. Despite these requirements, 2013 EHDI statistics from the CDC (n.d.-a, n.d.-b) indicate that only $63.9 \%$ of the children identified as deaf or hard of hearing are currently enrolled in early intervention services (Alam, 2016; Uhler et al., 2014).

Referral to early intervention should not be deferred until audiologic diagnostic evaluation and hearing aid fitting are completed. For all infants who are confirmed as being deaf or hard of hearing, either unilateral or permanent bilateral in type, a referral to early intervention must be made. Reports should also go to the infant's medical home, primary care provider, pediatric otologic physician (if known), and to the state EHDI program. The infant's primary care provider should be kept apprised of all findings as evaluations and early interventions continue.

\section{Early Intervention: Services for Infants/Toddlers from Birth to Age Three Years and their Families}

Some states currently characterize their birth-to-three programs as early development programs rather than early intervention programs. For the 2019 statement, the $\mathrm{JClH}$ made a decision to retain the term early intervention, because it is referenced in federal law and is familiar to readers. However, it is suggested that future consideration be given to using a term like early development that captures the proactive and capacitybuilding nature of the intervention with identified infants, toddlers, and young children and their families.

There is growing evidence that infants who are identified early in life and provided with timely early interventions demonstrate better linguistic outcomes than later-identified infants and children. These effects have been observed in vocabulary development (Mayne, Yoshinaga-Itano, Sedey, \& Carey, 1998), receptive language (Kennedy et al., 2006; Vohr et al., 2012; Watkin et al., 2007; Yoshinaga-Itano et al., 2010), expressive language (Pipp-Siegel, Sedey, VanLeeuwen, \& Yoshinaga-Itano, 2003; Yoshinaga-Itano et al., 2010), syntax (Yoshinaga-Itano et al., 2010), speech production (Ambrose, Unflat Berry, et al., 2014; Yoshinaga-Itano, Coulter, \& Thomson, 2000) and socialemotional development (Pipp-Siegel, Sedey, \& YoshinagaItano, 2002; Yoshinaga-Itano, Sedey, Coulter, \& Mehl, 1998). Tomblin and colleagues (Tomblin et al., 2014; Tomblin et al., 2015) demonstrated a positive impact on longitudinal language growth trajectories when amplification was provided early for children who are hard of hearing.

Selected population-based studies from the past 10 years did not find an association between age of identification and later child outcomes (Fitzpatrick et al., 2007; Geers et al., 2009). In part, these differing results may reflect the fact that the distributions for age of identification are quite distinct for groups studied prior to and after the implementation of newborn hearing screening (McCreery, Walker, et al., 2015). In addition, the reasons for late identification may differ in the pre- and post-newborn hearing screening contexts, which further impacts the respective study populations (McCreery, Walker, et al., 2015). Additionally, for deaf children with cochlear implants, age at implantation has been found to be a stronger predictor of spoken language outcomes than age at identification (Geers et al., 2009; Ching, Leigh, \& Dillon, 2013). 
Providing children and families the earliest possible start in intervention is considered best practice (Joint Committee on Infant Hearing, 2013; Moeller et al., 2013). Early interventions are widely recognized as a means to provide infants access to linguistic input, whether through spoken and/or sign language. Access to linguistic input is regarded as essential for successful communication and language development. Data from hearing children show that simply being exposed to more words is a very potent factor driving communication development (Hart \& Risley, 1995; Hurtado, 2009; Suskind et al., 2013). This practice of providing early access is supported by recent longitudinal studies, showing the positive influence of early ages at device fitting and duration of device use on children's outcomes (Nicholas \& Geers, 2007; Sininger et al., 2010; Tomblin et al., 2014, 2015). In spite of improving outcomes for children who are deaf or hard of hearing, selected epidemiological studies suggest that, on average, some children remain at risk for delays in spoken language, even when identification is early (Tomblin et al., 2015; Ching et al., 2013).

Early interventions are designed to minimize or prevent such delays and to promote linguistic development and access through high quality communicative interactions in the home. To accomplish this, early interventions are designed to promote caregiver use of language facilitation strategies. Several studies indicate a linkage between parental communication and child language outcomes. For example, large-scale investigations of parent-child interactions demonstrated the positive influence of caregiver use of language-promoting strategies on child language outcomes (Cruz, Quittner, Marker, \& DesJardin, 2013; Quittner et al., 2013) and the importance of avoidance of overly directive interactions (Szagun \& Schramm, 2016; Ambrose, Vandam, \& Moeller, 2014). Szagun and Schramm documented the strong influence of the home linguistic environment on development in young children who are deaf. Child language outcomes were also found to be enhanced when caregivers engaged their toddlers who were hard of hearing in frequent conversational turns (Ambrose, Vandam, \& Moeller, 2014; Hoffman, Quittner, \& Cejas, 2015). Hoffman and colleagues found exposing deaf children early to language has a significant and positive impact on children's social competence.

Several family and child background factors have been linked with child outcomes, including absence of additional disabilities, severity of hearing thresholds, gender, and maternal education and age at activation of cochlear implants (Geers et al., 2009). Higher levels of family involvement in promoting children's communication are associated with enhanced child language outcomes (Watkin et al., 2007). Greater maternal stress was associated with greater risk for vocabulary delays and for child behavioral challenges (Topol et al., 2011). Although more research is needed, enhanced maternal selfefficacy may be a particularly beneficial family outcome from early intervention (DesJardin \& Eisenberg, 2007;
Stika et al, 2015). Collectively, these results support the value of providing early access to services to ensure that language and communication development is optimized for the child and family.

It is widely recognized that a key component of providing quality services is the expertise of the provider, including knowledge and skills that are specific to working with children who are deaf or hard of hearing and their families (JClH, 2013; Moeller et al., 2013). These services may be provided in the home or in a center, in a combination of the two locations, or in community-based environments. However, home-based services were found to be associated with higher levels of family participation than non-home-based locations (Harrison et al., 2016).

\section{Family-Centered Practices}

The Early Childhood Technical Assistance Center (ECTAC; 2016) describes family-centered principles as a set of interconnected beliefs and attitudes that shape directions of program philosophy and behavior of personnel as they organize and deliver services to children and families. In 2014, the Division for Early Childhood, Council for Exceptional Children published recommended practices for early intervention. Sensitivity and respect for the culture and values of individual family members and each family's ecology is the core of these services, as members define the people, activities, and beliefs important to them. The definitions of family-centered care from different fields and disciplines often include these common descriptors: strengths-based, consumer-driven, family systems, family support, empowerment, proactive service delivery, competency-focused, partnerships, collaborative relationships, and family-driven (Dunst, Trivette, \& Hamby, 2007; Dunst \& Dempsey, 2007).

All of these general principles apply when the family has an infant/toddler who is deaf or hard of hearing. As partners, the family and professionals often place primary emphasis on communication and language access for the infant/toddler, and on provision of a language rich environment throughout daily routines (Quittner et al., 2013; DesJardin, Ambrose, \& Eisenberg, 2009). A primary goal of the early intervention program is to foster families' abilities to provide an abundance of ageappropriate language opportunities, joint engagement routines, and regular conversational turns throughout natural daily interactions. Families are supported in developing the confidence and competence to promote the infant/toddler's development through attunement to the child, providing an engaging social-emotional climate, and by creating and maximizing natural language and other learning opportunities. Families gain an understanding that their efforts to stimulate language development and provide language access in a time-sensitive manner are critical steps in addressing the needs of the child to optimize social, cognitive, and linguistic outcomes. The role of the professional in this enterprise is that of supporter, partner, and coach (Rush \& Shelden, 2011). 


\section{Goals for Early Intervention Programs}

In 2013, the JClH published a supplement to its 2007 Position Statement, entitled, "Principles and Guidelines for Early Intervention Following Confirmation That a Child Is Deaf or Hard of Hearing." The goals listed with details regarding the rationale, guidelines, and benchmarks for each can be found in the complete document. The supplement acknowledges that early intervention is a primary goal of the EHDI process. Best practices dictate the need for evidence-based, carefully designed, and individualized intervention, and for early education programs that are implemented promptly, using service providers with optimal knowledge and skill levels.

At the current time, there are major gaps in the evidence about the ways in which service provision interacts with child and family characteristics to influence outcomes (Eisenberg et al., 2007). In spite of limitations and gaps in the evidence, the literature does contain research studies in which all identified children had access to similar, well-defined early intervention services. These studies indicate that positive outcomes are possible, and they provide guidance about key program components that appear to promote these outcomes. The JCIH 2013 supplemental statement on early intervention was drafted by a team of professionals with extensive expertise in early intervention programs for children who are deaf or hard of hearing and their families. These early intervention experts relied on extensive literature searches and review of current studies, existing systematic reviews, and recent professional consensus statements in developing goals listed below and related best practice guidance (Centre for Allied Health, 2008). Twelve best practice goals for early intervention may be found in the $\mathrm{JClH} 2013$ supplement on early intervention.

\section{Supporting families in the process of informed decision-making. Families of infants and toddlers who} are deaf or hard of hearing encounter new concepts and technical information very early in the intervention process, and they often need to make a number of decisions (e.g., about amplification, languages, and modalities) leading to communicative competence at key stages of the child's development. Informed decision-making is a fluid and ongoing process that requires a family to consider new knowledge, information, experiences, language assessment results, and their personal family goals and values as they make decisions. Service providers must strive to collaborate and work together with families on the process of gaining the necessary knowledge, information, clarity, and experiences that support fully-informed decisions. Elements that impact a family's decisions include their child's unique strengths and abilities, their family's value and belief system, social circumstances, cultural influences, and personal comfort level in making choices on behalf of the child.

Young and colleagues (2006) emphasize that informed choice is not synonymous with the provision of information that is neutral or functionally descriptive. Rather, evaluative information is essential in that it draws attention to the various benefits, risks, and family responsibilities that are associated with particular decisions and/or choices. This suggests that it is incumbent upon professionals and family support programs to provide comprehensive guidance about expected child outcomes as well as what the family can expect to commit to when selecting specific intervention approaches or devices. Ultimately, decision-making authority rests with the family (Dunst, Trivette, \& Deal, 1988). Collaborations with professionals support families' abilities to exercise this authority. The Division for Early Childhood of the Council for Exceptional Children (2015) published checklists to assist early intervention providers in assessment of services for family centeredness, family engagement, informed decision-making, and family capacity building.

Each family should be fully informed about various approach(es) to language and communicative development, and should be fully informed of the necessity for promotion of language(s) acquisition at the earliest possible time following identification. Families should be encouraged to consult with their early intervention team, other specialists (e.g., audiologists, speech-language pathologists, ASL or other sign language specialists, adults who are deaf or hard of hearing, physicians), their child's health care provider, and other parents of children who are deaf or hard of hearing throughout the decision-making process. It is beyond the scope of the current $\mathrm{JCIH}$ document to provide comprehensive coverage of the various options to promote language access. Readers are referred to the 2013 Supplement to the JClH 2007 document for further guidance.

Supporting family and infant mental health. Early intervention providers work with a diverse array of families who have a range of individual needs, circumstances, and resources. The early identification period of learning, gathering resources, and making decisions is naturally stressful for families. However, concerns arise if elevated life stressors persist at a level that creates parenting challenges that may impede natural bonding and acceptance of the child being deaf or hard of hearing. Previous studies find that, on average, hearing parents of children who are deaf or hard of hearing do not report elevated levels of stress in general aspects of parenting (Åsberg, Vogel, \& Bowers, 2007; Dirks, Uilenburg, \& Rieffe, 2016; Meadow-Orlans, 1994). However, some families may be at risk for context-specific stressors related to the child being deaf or hard of hearing. These may include communication challenges and educational concerns, behavioral issues, maintaining use of visual and auditory devices, receiving several different types of services, and ensuring that family members and teachers are communicating effectively with the child using visual and/or spoken language (Hintermair, 2006). High levels of parenting stress may affect outcomes for children who are deaf and parental access to personal and social resources reduces stress (Hintermair, 2006). 
For some families, especially those living with extreme stressors (e.g., poverty, depression), mental health concerns have the potential to disrupt healthy mother and infant or child attachment and interventions focused on maternal sensitivity may be advisable. Maternal stress and depression have been linked to levels of mother-child attachment security (Atkinson et al., 2000). Infant Mental Health specialists and Home Visiting programs (Filene, Kaminski, \& Valle, 2013) may be a useful resource to EHDI teams when families are dealing with significant and/ or lingering and unresolved life stressors. See the World Association for Infant Mental Health (http://waimh.org) and the HomVEE (https://homvee.acf.hhs.gov/) websites for more information.

EHDI programs need to ensure that individualized family supports are accessible (Jackson, 2011), and that teams of deaf, hard of hearing, and hearing El providers have training to promote parental sensitivity and adjustment while preparing families to develop positive self-concept in the child who is deaf or hard of hearing. These supporting factors can empower families to learn more about being deaf and begin to make key decisions and take actions that will fully support the child's self-concept and learning. Ongoing surveillance and further research are needed related to supporting family and infant mental health.

\section{Supporting a new generation of families. Modern} families take on many forms and may involve multiple generations. Early intervention programs must be flexible in identifying ways to involve family members and support systems that play key roles in the infant and young child's life. Today's generation of families tend to access information through the on-line resources, including both from trustworthy resources or potentially unreliable sources of information. They may engage in opinion sharing through social media. Early intervention programs need to identify and respond to families' preferred modes for accessing information, guiding them toward reliable sources and networking with one another. To support family members in gathering information to make informed decisions, attention must be given to adult learning theories, recognizing that adults do not learn the same way that children learn. Professionals must become familiar with basic theories and principles of adult learning and with learning styles present among multigenerational dynamics (Kuhn \& Pease, 2006).

Supporting families during service transitions. As more children who are deaf or hard of hearing are identified early in life, service transitions may be happening at different times based on the quality of the early services provided, the progress of the individual child, and family circumstances (Walker et al., 2014). The most common transition following early intervention birth-to-three services happens when the child turns three years of age and services are then transitioned from IDEA Part C (early intervention support services) to Part B (school-aged) services, or to the education system in their geographic region (U.S. Department of Education, n.d.-a). In general, the transitional support services provided by professionals should be smooth, informative to caregivers, familycentered, child-focused, and assessment-based. Bridge to Preschool is a publication modeling an effective process for helping families through this transition (Colorado School for the Deaf and the Blind, 2011). Resources for supporting transitions from Part C to Part $B$ are also available from the Early Childhood Technical Assistance Center website (ECTAC, n.d.).

Smooth transitions are best supported by the professional who knows the family well, with the processes beginning well ahead of the anticipated transition date. According to federal law, a transition must begin by at least six months prior to the child's third birthday. Hands \& Voices, a national parent driven organization dedicated to supporting families of children who are deaf or hard of hearing, has recommended that several key topics should be discussed during home visits as early as twelve months before the child's third birthday (Johnson, 2001). Such topics may include educational placements and timelines, identifying possible preschools, discussing the child's learning styles and mode(s) of communication with regard to group settings, meeting other families of preschoolers, and other pertinent family-identified questions. The familyinfant specialist/home visit provider is best equipped to provide these initial discussions as they are likely to be the professionals who know the family best and can provide initial professional opinions on potential preschool placements. A specific transition plan that considers eligibility for services and Individual Family Services Plan (IFSP) and Individualized Education Program (IEP) differences should be completed prior to the third birthday. The IEP and transition portfolio should be completed on or before the child's third birthday. If a child has equipment (including hearing aids, cochlear implants, visual technology and/or FM systems) to support his or her learning or communication, the proper use of that equipment should be documented and monitored as part of the preschool transition plan to ensure that the child can continue to build language and learn in his or her new setting.

A transition that is both family centered and informative to families carefully considers the family's observations and goals for their child, and also provides the family with information to make sound decisions about transition. Families must understand placement opportunities and the ways in which they address the communication access needs of the child, assessment information, what to expect in the next phase, recommended opportunities for services, and the differences between an IFSP and an IEP. It is important to ensure that the needs of children who transition with age-appropriate language and communicative skills continue to be monitored and supported. Children who are maintaining their trajectory of developmental growth through preschool will have at minimum: proper hearing assistance technology and/or visual technology use; articulation support for spoken language users who qualify; appropriate interpretation services and/or direct instruction in sign language (e.g., ASL for ASL users); family education and resources, such 
as Deaf mentors, ASL for families; quarterly

educational audiology support services; language and academic growth monitoring at least every six months; and mainstream itinerant observation or itinerant child/teacher support as part of the preschool transition plan.

\section{Supporting families through innovative models of service delivery: Tele-practice. Tele-practice can be} broadly defined as the use of technology to deliver professional services such as parent education, assessment, and intervention over a geographical distance (Behl \& Kahn, 2015). Families who live far from qualified providers, or who do not have the means to travel, may encounter challenges in accessing selected specialized early intervention services. Modern telecommunication technology has the potential to overcome those obstacles, but further research is needed. Although tele-practice programs have emerged nationally and internationally, there is a limited body of peer-reviewed empirical evidence in the context of early intervention for families of children who are deaf or hard of hearing. Some evidence exists in relation to Audiological and $\mathrm{Cl}$ practices, although most of this work has focused on adults. Swanepoel and Hall (Swanepoel \& Hall, 2010) conducted a systematic review of 26 peer-reviewed empirical studies of tele-practice applications in Audiology. They concluded that initial reports are promising, but that studies are limited in breadth and depth, suggesting a pressing need for more research to overcome barriers to practice application. A few studies have empirically evaluated applications of tele-practice for $\mathrm{Cl}$ service delivery (Hughes et al., 2012; McElveen et al., 2010). However, studies of the feasibility of tele-practice for $\mathrm{Cl}$ interventions with young children are lacking. A recent survey (Behl \& Kahn, 2015) suggests that practitioners are using tele-practice to supplement face-toface services, and that there is a need to address challenges in internet connectivity and professionals' training in delivery of tele-practice services for early intervention. Tele-practice applications may be helpful for connecting families with needed supports, such as ASL training, coaching to promote spoken language development, and family to family connections.

\section{Language Assessment and Intervention}

Being deaf or hard of hearing occurs in people of all nationalities, all cultural heritages, and from all language backgrounds (Crowe, Mcleod, \& Ching, 2012). This cultural and linguistic diversity is often attributed to the family and community in which they live (Crowe et al., 2012). Recognition of the cultural and linguistic diversity of young children who are deaf or hard of hearing is critical to planning and providing assessment, habilitation, and education services to both children and their families (Crowe, McKinnon, McLeod, \& Ching, 2013). In the ever-increasing diversity of linguistic environments of today's society, the language of the home must be taken into consideration and viewed as the foundation from which to build.
The process of language development includes learning the precursors of language, such as the rules pertaining to selective attention and turn taking (Gerken \& Aslin, 2005). Cognitive, social, and emotional development are influenced by the acquisition of language (Landry, Smith, \& Swank, 2006). Development in these areas is synergistic. A complete language evaluation should be performed at regular intervals for infants and toddlers who are deaf or hard of hearing. Assessment conducted by a provider who is fluent in the language of the home is known to increase the reliability and validity of the assessment (Caesar \& Kohler, 2007). The evaluation should be done by qualified professionals and include an assessment of spoken or signed language development in the child's dominant language (as chosen by the parents), as well as an assessment of the observable abilities of the child.

A primary focus of Part $C$ services is to support families in fostering the communication abilities of their infants and toddlers who are deaf or hard of hearing. Spoken and/ or sign language developmental trajectories should be commensurate with the child's age and cognitive abilities, and should include acquisition of phonologic, morphologic, semantic, syntactic, and pragmatic skills for signed and/or spoken language (Meinzen-Derr, Wiley, Grether, \& Choo, 2011). If developmental goals are not being met, timely adjustment in the intervention plan or communication approach needs to be considered.

Families should be provided with information specific to language development and access to peer and language models. Families also benefit from family-involved activities that facilitate language development of hearing children and children who are deaf or hard of hearing (JClH, 2013; Moeller et al., 2013). Families should be offered access to children and adults who are deaf or hard of hearing, as role models, mentors, and as appropriate and competent language models (Watkins, Pittman, \& Walden, 1998). Access to deaf or hard of hearing adults can support caregivers in fostering the child's emerging self-esteem and identity formation. Information on spoken language, signed language, or use of both should be provided (JClH, 2013; Moeller et al., 2013).

\section{Medical Home and Ongoing Surveillance}

Regardless of previous hearing-screening outcomes, all infants with or without risk factors should receive ongoing surveillance of communicative development beginning at 2 months of age during well-child visits in the medical home (AAP Committee, 2017). This recommendation provides an alternative, more inclusive strategy of surveillance of all children within the medical home based on the pediatric periodicity schedule (AAP Committee, 2017; AAP, 2014a). All Infants who do not pass the speech-language portion of a medical home global screening or for whom there is a concern regarding hearing or language should be referred for speech-language evaluation and audiology 
assessment. This protocol permits the detection of children with either missed neonatal or delayed-onset hearing loss, irrespective of the presence or absence of a high-risk indicator.

\section{Ongoing and Continuous Surveillance, Screening, and Referral of Infants and Toddlers}

To this point, the $2019 \mathrm{JCIH}$ Position Statement has outlined the sequence of events for screening the hearing of all newborns, providing prompt audiology evaluation for those who do not pass newborn screening, and offering timely intervention for identified infants. However, the child who has a passing result on newborn hearing screening may develop, or show evidence of, childhood hearing loss. If one to two infants out of every thousand are diagnosed as deaf or hard of hearing at birth, it is estimated that another one to two per thousand will later be diagnosed with permanent hearing loss (Mehra, Eavey, \& Keamy, 2009). This may reflect delayed-onset hearing loss as well as missed conductive, sensory, or neural hearing loss at the time of newborn hearing screen. Infants with minimal/ mild hearing loss are likely to pass newborn screening. The rate of being deaf or hard of hearing is known to increase from approximately $1.2 / 1000$ in newborns to $3 / 1000$ in early school age. In a recent report (Watkin \& Baldwin, 2012), the prevalence of children confirmed as deaf or hard of hearing by school age was 3.65/1000 compared to a neonatal yield of $1.79 / 1000$.

For these reasons, all children should receive surveillance of speech and language milestones and auditory responsiveness in the medical home (AAP Committee, 2017). Continuing efforts to inform and educate primary care providers about the importance of ongoing surveillance and screening are encouraged. In addition, programs and resources that inform and educate families and caregivers about typical auditory development and about typical spoken and/or signed language development can result in more rapid identification of delayed-onset or progressive hearing loss and/or fluctuating hearing thresholds. Families can be encouraged to seek evaluation should they have concerns about their child's progress. Education of the families and caregivers about typical language development (spoken and/or signed) and how to encourage and facilitate language growth in their child would also foster earlier identification of delayed-onset or progressive hearing loss, or otherwise unidentified elevated hearing threshold levels.

\section{Surveillance and Rescreening for Children with Risk Factors}

Risk factor information should be collected, stored, and easily accessible in the electronic medical record, since the presence of risk factors places the infant at increased risk of delayed-onset hearing loss, regardless of the newborn hearing screen results.

The majority of all infants identified as deaf or hard of hearing will be followed closely by an audiologist and otolaryngologist and receive indicated intervention and support services. However, a significant number of children will pass the newborn screen or rescreen who are at risk for later-onset hearing loss. Table 1 shows a revised list of risk factors since the $2007 \mathrm{JCIH}$ statement. The recommendations for follow-up and evaluation on this list of risk factors pertain to infants who pass the newborn screen or rescreen. These recommendations for follow-up are based on the fact that standard newborn screening procedures and protocols do not identify all children who are deaf or hard of hearing due to missed mild or neural hearing loss, progressive hearing loss, and delayed-onset hearing loss (Johnson, 2005a; Walker et al., 2014; Nance, 2003). As shown in Table 1, the prior eleven risk factors listed in $\mathrm{JCIH} 2007$ are now listed as 12 separate factors and divided into subgroups of predominantly perinatal (risk factors 1-9) and perinatal or postnatal (risk factors 10-12). In addition, the recommendations for follow-up and monitoring have been modified and are described in greater detail, and the age of audiologic diagnostic follow-up for children with risk factors who passed newborn hearing screening has been lowered for most risk factors to by 9 months of age. Supporting evidence for the revised $\mathrm{JClH}$ risk factor recommendations is provided in the following sections.

Perinatal Risk Factors. The first nine risk factors occur solely in the perinatal period.

Risk Factor 1. A history of family members being deaf or hard of hearing with onset in childhood, has consistently been shown to be predictive that the diagnosis is secondary to a spectrum of genetic causes, and therefore stands alone as a particularly concerning risk factor. Monitoring continues to be based on both the etiology and the level of family concern, with a diagnostic evaluation recommended by 9 months of age (NIDCD, 2002; Morton \& Nance, 2006; Santos et al., 2005; Dedhia, Kitsko, Sabo, $\&$ Chi, 2013) or earlier if parent or caregiver concern is expressed (Dedhia et al., 2013). During the child's newborn period, some parents may not be aware of a family history, as this information may be shared by relatives only after the infant has not passed the hearing screen or diagnostic testing.

Risk Factor 2. Infants who require care in the NICU or special care nursery for more than five days is used as an indicator of illness severity $(\mathrm{JClH}, 2007)$. Although there are a growing number of reports addressing NICU noise exposure, such noise exposure has not been included as a separate category, since it is included in the classification of more than 5 days in the NICU (Daniell et al., 2006; Lasky \& Williams, 2009).

Risk Factor 3. Hyperbilirubinemia as a risk factor for hearing loss is impacted by multiple factors including illness severity, birth weight, rate of rise of bilirubin, clinical findings, postnatal age of the infant, and gestational age, as all premature infants have some degree of 
hyperbilirubinemia. Management of these infants should be based on established protocols (Morris et al., 2008). Hyperbilirubinemia requiring exchange transfusion is the only risk factor for which close follow-up is recommended regardless of length of stay in the NICU (Oh et al., 2003; Shapiro, 2003; Wickremasinghe et al., 2015). This exception is made since some term infants requiring exchange transfusion may be discharged before 5 days in the NICU.

Risk Factor 4. Aminoglycoside administration of more than 5 days is reaffirmed; administration of aminoglycoside antibiotics for less than 5 days is considered a risk factor only if toxic blood levels are identified or if there is a family history of a mitochondrial genetic mutation associated with aminoglycoside sensitivity for sensorineural hearing loss. Several mitochondrial DNA mutations of the 12S rRNA gene are known to be associated with an increased risk of aminoglycoside-induced hearing loss. However, aminoglycosides are one of the most common medications administered in the NICU (Clark, Bloom, Spitzer, \& Gerstmann, 2006). Studies have examined the frequency of the mitochondrial gene mutations in NICU populations and identified a rate of approximately $1-1.8 \%$ (Ealy, Lynch, Meyer, \& Smith, 2011; Johnson, Cohen, Guo, Schibler, \& Greinwald, 2010). In the study by Johnson and colleagues, $87 \%$ of infants admitted to the NICU received aminoglycoside treatment for between 1 and 24 days and of those, $0.9 \%$ had a mitochondrial mutation and received aminoglycosides. Ten patients did not pass the repeat $A B R$, but only a single infant with a mitochondrial mutation that received gentamicin developed hearing loss, and this child was extremely low birth weight. Since three children with the mutation did not develop decreased hearing, the authors speculate on the potential existence of a modifier gene that conferred a protective effect. Nevertheless, low birth weight was the primary risk factor for atypical hearing thresholds in this study, and the duration of gentamicin exposure for NICU infants with atypical hearing thresholds was 5.44 days as compared to 3.92 days for those with hearing within the typical range. Ealy and colleagues genotyped NICU patients and adults from the general population and identified that $1.8 \%$ of both populations had one of the mitochondrial DNA variants. They identified no association between the mitochondrial variant and atypical hearing thresholds in the NICU population.

Since approximately 400,000 infants per year are cared for in a NICU in the United States and the majority have at least 1 day of aminoglycoside exposure, follow-up diagnostic audiology assessments on all of these infants is not indicated. Based on these findings, it is recommended that infants who received care in the NICU, who passed the newborn hearing screen, and who received aminoglycosides for less than or equal to 5 days, receive standard surveillance in the medical home of hearing and spoken language milestones following the recommendations of the American Academy of Pediatrics periodicity schedule (AAP Committee, 2017). The recommendations may change as multi-gene panels become standard in newborn screening. There are no clinical indications to delay screening for eligible infants who have had aminoglycosides administered, including those infants who received 5 days or less, infants who received more than 5 days, and infants who may continue on aminoglycosides at the time of discharge.

Risk Factor 5. Perinatal asphyxia, also termed hypoxic ischemic encephalopathy, particularly if requiring hypothermia treatment, is noted, because of the illness severity of this sub-group of infants and the significant increase in permanently elevated hearing thresholds (Shankaran et al., 2012).

Risk Factor 6. Extracorporeal membrane oxygenation (ECMO) is specifically noted because of the increased risk of delayed-onset hearing loss (Lasky, Wiorek, \& Becker, 1998; Shankaran et al., 2012; Robertson, 1995).

Risk Factor 7. In-utero infections (i.e., herpes, rubella, syphilis, and toxoplasmosis) pose a risk and require follow-up by 9 months of age. cCMV infection is a leading cause of congenital infection occurring in 0.2 to $2 \%$ of live births world-wide and is a leading cause of non-genetic unilateral or bilateral early, progressive, and delayed onset sensorineural hearing loss (Harrison et al., 2016; Dunst et al., 2007; Dunst \& Dempsey, 2007). Therefore the recommendation for follow-up audiologic assessment of infants with CCMV is no later than 3 months of age. Zika virus has been added to the list of in-utero infections associated with hearing loss. In 2016 the CDC (2016a) issued a series of interim guidance statements for the caring of infants with possible congenital Zika virus infection which included recommendations for hearing assessment (Staples et al., 2016; Fleming-Dutra et al., 2016; Russell et al., 2016; Leal, Muniz, Ferreira, et al., 2016; Leal, Muñiz, Caldas Neto, et al., 2016). In October 2017 the interim guidance was updated in recognition of the absence of data suggesting delayed-onset hearing loss following congenital Zika virus infection (Adebanjo et al., 2017). The updated guidance suggests infants born to mothers with possible Zika virus exposure during pregnancy or with findings consistent with congenital Zika syndrome should receive a standard newborn screen at birth, preferably using an ABR methodology (Adebanjo et al., 2017). These infants should be referred for automated $A B R$ by age 1 month if the newborn hearing screen was passed using only OAE methodology.

Risk Factor 8. All craniofacial conditions and physical conditions associated with hearing loss are now included as the 8th risk factor (formerly risk factors 5 and 6 ).

Risk Factor 9. More than 400 syndromes and genetic disorders associated with atypical hearing thresholds (formerly risk factors 7 and 8) are now included as the 9th risk factor (Morton \& Nance, 2006; Santos et al., 2005; Van Camp \& Smith, 2017). 
Perinatal or Postnatal Risk Factors. The remainder of the risk factors may occur either in the perinatal or postnatal period.

Risk Factor 10. Perinatal and postnatal confirmed bacterial and/or viral meningitis or encephalitis (especially herpes viruses and varicella and hemophilus influenza and pneumococcal meningitis are included in the 10th risk factor (Rubin \& Papsin, 2010).

Risk Factor 11. Predominantly postnatal events of significant head trauma particularly injury to the mastoid (Bergemalm, 2003; Zimmerman, Ganzel, Windmill, Nazar, \& Phillips, 1993) and chemotherapy (Gruss, Handzel, Ingber, \& Beiser, 2012) comprise the 11th risk factor category.

Risk Factor 12. Family/caregiver concern regarding hearing, speech, language, or development requires attention. The recommendation is immediate referral after discussion with family. One of the most important risk factors is family/caregiver concern regarding hearing. When a caregiver expresses concern that the baby is not responsive to sound in the home environment, or about speech and/or language delays, it is recommended that the primary care provider obtain additional medical and language history and evaluate the child carefully for middle ear effusion. Referral to a pediatric audiologist to evaluate hearing is recommended regardless of risk factors and possible presence of middle ear effusion.

When risk factors for delayed-onset or progressive hearing loss are present, comprehensive audiologic evaluation should occur (depending on the risk factors) in the period between hospital discharge and 9 months of age. The schedule for on-going re-evaluation is predicated upon both the specific risk factors and the observations by the family of their child's auditory and speech/language development. In addition, for hospital readmissions in the first month of life when there are conditions associated with potential elevated hearing thresholds (e.g., hyperbilirubinemia or culture-positive sepsis), an AABR screening should be performed before discharge even if the baby has passed newborn hearing screening prior to the development of the condition requiring re-admission.

\section{Surveillance and Periodic Rescreening of Hearing in the Early Childhood Years}

Careful surveillance of hearing throughout the early childhood years up until and including kindergarten entry (continuous childhood screening), even in the absence of known risk factors for hearing loss is recommended, since the prevalence may double by school-age (NIDCD, 2005; Niskar et al., 1998). Hearing screening may be achieved through OAE screening in the medical home or other pre-school settings up to age three, and use of pure tone audiometry screening in the medical home along with a developmental checklist for speech and language milestone assessment is appropriate throughout early childhood.
These screening options may be provided by the child's medical home provider during regularly-scheduled well-child visits (AAP Committee, 2017). There should be a designated pathway for referral to a pediatric audiologist when concerns regarding hearing and/or speech-language development arise. Hearing screens or languagedevelopmental checklists may also be provided through infant and preschool programs.

\section{Consideration of Universal Preschool Hearing Screening}

There is a growing body of research that is beginning to better define the prevalence of delayed-onset hearing loss in the preschool population. Bhatia, Mintz, Hecht, Deavenport, and Kuo (2013) reported results of a mass screening program of 1,965 toddlers who were seen in participating medical clinics. In an OAE screening program offered to this population of children ranging between zero and three years of age, the authors were able to identify $5 / 1965$ patients $(0.25 \%)$ with permanent sensorineural hearing loss. Eiserman and colleagues (2008) also published results from OAE screening tests performed on children in a preschool setting. In this cohort, they were able to diagnose $7 / 4519$ children $(0.15 \%)$ who were deaf or hard of hearing. In a 2007 study published in Early Childhood Research Quarterly, Eiserman and colleagues reported on an OAE screening program of combined pre-school testing efforts. Overall, 6/3486 children (0.17\%) were eventually diagnosed as deaf or hard of hearing.

Even if a few of the identified children in these three studies might have actually represented children missed at birth, it appears that the prevalence of delayed-onset hearing loss during preschool years may very well merit consideration of mass screening programs. However, the prevalence of delayed-onset preschool hearing loss is only one of several criteria to consider in the process of justifying a population-based preschool screening program.

Screening during preschool years is complicated by the incidence of occult middle ear effusions associated with minor upper respiratory illness. Such asymptomatic effusions commonly resolve spontaneously and typically require no intervention, but will complicate the screening process as false positive test results requiring follow-up. False positive rates in preschool screening programs have been reported as high as $6-10 \%$ or more, in part due to these asymptomatic middle ear effusions, and significantly greater than the $1-4 \%$ false positive rates of most hospital-based newborn screening programs (Hall, 2016).

Also contributing to the false positive rate is the challenge of securing the cooperation of the preschool-aged child to complete physiologic (OAE) testing. If the ideal age for proposed universal preschool screening is in the area of 18-24 months, this same age would also be acknowledged to be a time when young children are typically the least cooperative with medical procedures or testing. Additionally, false negative results with $\mathrm{OAE}$ 
screening programs are a continuing concern. Due to the limitations of hearing screening with this technology, OAE testing may not identify children with mildly elevated hearing levels 25 to $40 \mathrm{~dB} \mathrm{HL}$ (Johnson, 2005a).

The feasibility of providing universal preschool-aged hearing screening is also an important limiting factor. UNHS has been a successful initiative in part because almost all infants are born in a hospital, and hospital-based programs therefore have the potential of capturing 95 percent or more of all newborns in a screening program (Winston-Gerson \& Hoffman, 2017). Similarly, the great majority of children enter a public school system around age five, and therefore school-based programs have demonstrated similar successes at screening the hearing of an entire population. However, during preschool years there is no similar common door through which almost all of the children pass.

The physician's office is a setting that potentially would capture most preschool-aged children for a mass screening program (at an age range to be determined), but not all children receive medical care in a timely way, due to a multiplicity of factors related to families and systems of care (Oberg, Colianni, \& King-Schultz, 2016). Rather than screening in the medical office, hearing screening in pre-school facilities or through home visiting programs could be an alternative mechanism, and such programs have been demonstrated with Head Start and through other preschool hearing screening initiatives (Council on Community Pediatrics, 2009). Clearly, a significant portion of the population of preschoolers in the United States may be enrolled, at least at intervals, in larger daycare or preschool settings. However, the proportion of such enrolled children in the overall preschool population would be far less than the $95 \%$ of newborns who are born in a hospital, and so the success of a proposed universal hearing screening program in this setting would be limited.

While acknowledging the concern about delayedonset hearing loss presenting during preschool years, the $\mathrm{JClH}$ finds that there is not adequate data to presently justify a broader recommendation for universal hearing screening during the preschool years. Further research and technologic advances may allow for an expanded recommendation in the future. Continued surveillance of language development by the family, caretakers, and the primary care provider, as well as observations of the child's responsiveness to auditory stimuli, is essential for recognition and timely diagnosis of delayed-onset hearing loss during preschool years.

\section{Protecting the Rights of Infants/Toddlers and Families}

Every stakeholder involved in the EHDI process shares responsibility for protecting rights and preserving confidentiality. Families should receive information about children who are deaf or hard of hearing and the potential benefits and risks of proposed interventions. The information should be presented in an easily-understood manner and whenever possible, in the family's preferred language. It is important that families have the right to accept or decline hearing screening tests or any follow-up care for their newborn infant within statutory regulations, just as they have such rights for any other screening or evaluation procedures or intervention.

Additionally, all EHDI data merit the same level of confidentiality and security afforded other health care and education information in practice and law. The infant's family has the right to confidentiality of the screening results and the follow-up assessments.

Three primary privacy regulations pertain to the exchange of EHDI information:

- Health Insurance Portability and Accountability Act (HIPAA) (U.S. Department of Health and Human Services, 2017)

- Family Educational Rights and Privacy Act (FERPA; U.S. Department of Education, n.d.-b)

- Part C regulations of IDEA (2004; U.S. Department of Education, n.d.-a; NCHAM, 2013)

The HHS Office for Civil Rights has responsibility for enforcing HIPAA rules with voluntary compliance activities and civil monetary penalties (U.S. Department of Health and Human Services, 2017). HIPAA regulations include both privacy and security rules. The Standards for Privacy of Individually Identifiable Health Information (Privacy Rule) establishes national standards for protected health information (PHI) in any medium (electronic, written, or oral) and establishes patient rights with respect to that information. The Privacy Rule permits PHI disclosure for necessary patient care and disclosure to public health and safety authorities. HIPAA regulations permit the sharing of health information among health care professionals, and the language in these regulations should facilitate the prompt audiologic and medical evaluation of an infant who does not pass the initial hearing screening tests.

Furthermore, the HIPAA Security Standards for the Protection of Electronic Protected Health Information (Security Rule) provides national security standards that must be put in place to secure individuals' electronic protected health information (e-PHI). The Security Rule specifies a series of additional administrative, physical, and electronic security practice safeguards to ensure the confidentiality, integrity, and availability of e-PHI, regardless of how they are delivered or accessed, including over the Internet. Under the HIPAA Security Rule, health care providers, hospitals, and clinics are required to implement policies and procedures to prevent, detect, contain, and correct security violations.

The NCHAM has several resources addressing the impact of privacy regulations (NCHAM, 2013) including a white paper "How EHDI, Part C, and Health Providers can Ensure that Children and Families Get Needed Services" (NCHAM, 2008). As noted in the report, strategies can be implemented to comply with signed consent requirement of Part $\mathrm{C}$ privacy regulations (which are more restrictive than FERPA) for the exchange of EHDI information. 
The recommendations of the workgroup who contributed to the report included:

- Provision of copies of diagnostic evaluation results, treatment plans, IFSPs, and any signed consent forms to families;

- Development of coordinated consent forms incorporating the elements required by HIPAA, FERPA, and Part C Privacy Regulations;

- Development of Memoranda of Agreement (MOA's) among EHDI, Part C, and Family Support Programs;

- Obtaining written consent from the family when newborns do not pass hearing screening or as part of the IFSP/IEP process;

- Development of state administrative rules and regulations or laws mandating the reporting of EHDI information to state programs and to the infant's medical home.

\section{EHDI Information Technology Infrastructure}

According to the Institute of Medicine (IOM), computerized clinical data and decision support systems are a prerequisite for the safe and comprehensive provision of quality care (Institute of Medicine, 2001). The IOM definition of quality is: "The degree to which health services for individuals and populations increase the likelihood of desired health outcomes and are consistent with current professional knowledge" (p. 232).

Overall, there have been improvements in the provision and documentation of screening and follow-up services in the United States, which have resulted in the early identification of more deaf and hard of hearing infants. This progress has been possible through improvements in the functionality of EHDI information systems and increases in the ability of EHDI programs to successfully track infants in need of follow-up services. However, some challenges remain in ensuring the receipt of follow-up services and additional efforts are needed to ensure all deaf and hard of hearing infants are identified early and receive intervention. These challenges include:

- Variation in the consistent reporting of follow-up data across EHDI programs, which impacts the completeness and quality of data;

- Differences in the infrastructure and capabilities of EHDI information systems, which limits the ability of some programs to accurately identify, match, collect, and report data on all births that is unduplicated and individually identifiable;

- Adoption of standard data definitions and varied performance measures among EHDI programs, potentially resulting in some differences in reported results;

- Understanding of data reporting requirements among providers and the burden to report data;

- Variation in the EHDI programs to measure and improve quality through continuous feedback and the application of computerized decision support; and

- Reliance to a great extent on 20th century measurement technology rather than 21st century technology (e.g., abstraction of samples of medical records and the reliance upon information from administrative datasets).

The JClH promotes an information technology infrastructure and national quality enhancement strategy focused on performance measurement of clinical quality and family satisfaction. The $\mathrm{JCIH}$ encourages further development, refinement, and pilot testing of well-defined EHDI performance measures, promotes the adoption and enhancement of EHDI information system infrastructure for comparing performance and disseminating results, and recommends a research agenda to support quality enhancement. The $\mathrm{JCIH}$ recognizes the need to address the issues most important to the provision of services and knowledge generation, while ensuring security safeguards for data, adequately protecting family privacy and limiting the burden of health data collection.

EHDI programs, whenever possible, should prepare for full implementation and adoption of nationally recognized standard data definitions and standardized measures to facilitate information exchange and analysis (Gaffney, Eichwald, Gaffney, \& Alam, 2014). In further refining their EHDI information systems, stakeholders should not reinvent the wheel, but rather build on and leverage work already underway in both the private and public sectors to establish a common conceptual framework for terminology definitions and standardized quality measures. In particular, electronic health data exchange standards for recording and transmitting newborn screening test results developed by the U. S. National Library of Medicine and child health quality measures endorsed by the National Quality Forum (NQF) should be adopted at the earliest possible time (CDC, n.d.-b; NQF, n.d.-a, n.d.-b, n.d.-c, n.d.-d). Resources for Early Hearing Detection and Intervention and Electronic Health Records Technology are available at the CDC website (CDC, n.d.-b).

The $\mathrm{JClH}$ recognizes the need to bolster the capacity and capabilities of EHDI programs for information exchange, ensuring that data collected in one system can be used by other systems for a variety of different uses (e.g., provision of services, quality assurance, research, and public health). Much of EHDI information exchange currently relies on paper forms that are mailed, emailed, or faxed, necessitating manual data entry and coding by the public health agency prior to initiating follow-up services, analysis, or reporting. The $\mathrm{JCIH}$ encourages programs and providers to migrate from paper-based health record systems to an information infrastructure that captures and stores data electronically and takes advantage of computer-aided decision support.

A functional foundation for an EHDI information system should have the ability to electronically collect, rather than manually enter, screening results and demographic information; accept, create, and report both clinical decision support and quality measures; and, leverage evolving local, regional, and national Health Information 
Exchange (HIE) capabilities. An EHDI information technology infrastructure needs to incorporate all aspects of quality enhancement, including the use of standardized measures and appropriate sharing of data where doing so will likely result in greater gains in the quality of services and reduce the burden on providers and families.

\section{Benchmarks and Quality Indicators}

The $\mathrm{JCIH}$ supports the concept of regular measurements of performance and recommends routine monitoring of these measures for inter-program comparison and continuous quality improvement. These performance benchmarks represent a consensus of expert opinion in the field of newborn hearing screening and intervention. The benchmarks are the minimal requirements that should be attained by high-quality EHDI programs. Frequent measures of quality permit prompt recognition and correction of any unstable component of the EHDI process.

\section{Quality Measurement and Improvement}

The provision of EHDI services can be improved and better coordinated when data are captured to measure performance and that information is shared among all stakeholders. Use of consensus-based standardized measures lessens reporting burden, focuses on a discrete targeted set of measures to improve services, and allows stakeholders to compare results.

To report and ensure information is accurate, complete, and transparent, all measures should have clear, unambiguous definitions for each numerator and denominator with well-defined exclusions/exceptions and data elements/value sets used for calculation. Whenever possible, nationally endorsed measures and standard data elements/coded value sets should be used. In addition, steps should be taken to measure and report individual-level geographic and demographic data.

National standard EHDI data elements/value sets are maintained and available for public use through:

- Agency for Healthcare Research and Quality (AHRQ, n.d.) U. S. Health Information Knowledge Base (USHIK)

- CDC (n.d.-c) Public Health Information Network (PHIN) Vocabulary Access and Distribution System (VADS)

- National Library of Medicine (NLM) Newborn Screening Coding and Terminology Guide (NLM, n.d.-a)

- NLM Value Set Authority Center (VSAC; NLM, n.d.-b)

EHDI has three measures endorsed by the NQF:

- Hearing screening prior to hospital discharge (NFQ1354) [NQF, n.d.-b]

- Audiological evaluation no later than 3 months of age (NFQ1360; NQF, n.d.-a)

- Intervention no later than 6 months of age (NFQ1361; NQF, n.d.-c]
These NQF measures were developed and are stewarded by CDC through a formal process for updating and maintenance. Additional EHDI quality measures need to ensure a rigorous and consensus-based process of development involving all stakeholders. To ensure accountability, individual, community, and state health and educational programs should assume the responsibility for coordinated, ongoing measurement and improvement of EHDI processes and developmental outcomes. As relevant quality measures are developed and communicated, stakeholder organizations should address what is important to achieve the best outcomes for deaf and hard-of-hearing children and their families, without creating an undue burden of data collection. Rather than promoting specific recommended benchmarks, $\mathrm{JCIH}$ strongly encourages the documentation of current baseline measurements and establishment of quality improvement activities for documenting continuous and measurable improvements in screening, confirmation of hearing status, and receipt of intervention services.

Quality indicators for screening. Quality indicators for newborn hearing screening are:

- Percentage of all newborn infants who complete screening by one month of age;

- Percentage of all newborn infants who do not pass initial hospital-based screening and require subsequent outpatient rescreening;

- Percentage of newborn infants who do not pass initial and any/all subsequent rescreening(s) prior to comprehensive audiologic evaluation; and

- Percentage of newborn infants who do not pass initial screening and subsequently pass a re-screening.

Quality indicators for confirmation that a child is deaf or hard of hearing. Quality indicators for confirmation of hearing status and diagnosis of hearing thresholds are:

- Percentage of infants who do not pass initial birth screening and any subsequent rescreening, and

- Percentage of infants who complete a comprehensive audiologic evaluation by three months of age.

For families who elect amplification:

- Percentage of deaf and hard of hearing infants receiving amplification devices within one month of confirmation of hearing status.

Quality indicators for early intervention. Quality indicators for early intervention for infants confirmed as deaf or hard of hearing and qualify for Part $C$ services include:

- Percentage of infants for whom parents have signed an IFSP no later than six months of age.

For children who are deaf or hard of hearing and have experienced late-identification or delayed-onset progression in hearing thresholds: 
- Percentage for whom parents have signed an individualized family service plan within forty-five days of the diagnosis.

- For all infants confirmed as deaf or hard of hearing:

- Percentage of infants who receive the first developmental assessment using standardized assessment protocols (not criterion reference checklists) in the language of the home for spoken and/ or visual language, speech, and nonverbal cognitive development no later than twelve months of age.

Effective January 1, 2016, The Joint Commission (TJC), formerly known as the Joint Commission on Accreditation of Healthcare Organizations (JCAHO), adopted the EHDI electronic Clinical Quality Measure (eCQM) for data reporting of newborn hearing screening before hospital discharge (Eichwald, 2016). Any accredited hospital may choose this measure as one of the six required sets to satisfy their accreditation and certification process. The 2016 Flexible Reporting Options can be accessed from TJC's website posted under the "Measurement" section, ORYX Performance Measurement Reporting (Joint Commission, n.d.). The Joint Commission accreditation is recognized by a majority of state governments in lieu of a hospital licensure inspection conducted by the state-licensing agency and by the Centers for Medicare and Medicaid Services in lieu of a Medicare certification survey, thus qualifying organizations to receive Medicare and Medicaid reimbursement.

\section{Future Directions}

In addition to the Benchmarks and Quality Indicators, best practices in the diagnosis and management of infants and children who are deaf or hard of hearing will continue to evolve in myriad ways, impacted by multiple factors. Future directions and goals for the EHDI process include the following points.

\section{Screening}

- Better definition of hearing levels that are identified, and missed, by current hearing screening technologies to define sensitivity and specificity of both AABR and OAE;

- Individualized in-ear calibration of the screening stimulus to improve sensitivity and specificity; and

- Consideration of universal preschool hearing screening, including cost and prognosis for improved outcomes.

\section{Diagnosis}

- Continued development of new technologies and stimuli to speed diagnosis and improve accuracy;

- Development of calibration standards for atypical stimuli used in infant diagnosis for all transducers, permitting better estimates of type and degree of hearing loss; and

- Continued increase in the number of audiologists with pediatric knowledge and skills with further reduction in the serious shortage of qualified providers.

\section{Amplification and Cochlear Implant Protocols and Technologies}

- Continued refinement in amplification, verification, and validation protocols to facilitate access to speech;

- Increased systems initiatives from state and local EHDI partners to support timely access and reduce barriers to hearing aid amplification;

- Increased monitoring of hearing aid usage through automated software programs that permit targeted parent-counseling;

- Increased number of children who are fit with hearing aid technologies, particularly those employing on-board wireless capabilities, designed to benefit all children who are fitted with amplification;

- Development of policies and procedures that result in increases in state-funding or third-party payers for amplification and other technologies;

- Improvement of protocols (preferably automated) and additional development of technologies to assess speech discrimination in children of all ages using physiological cortical auditory evoked potentials and behavioral procedures;

- Adherence to best practice evidence-based protocols for fitting pediatric-specific hearing aids to children;

- Expansion of criteria for cochlear implantation including lowered age of candidacy, as well as other options (unilateral hearing loss, hybrid-devices for children with substantial residual hearing) to provide earlier auditory access to a larger population of children.

\section{Healthcare Reform and Data Management Systems}

- Evidence supported by healthcare reform, improved data-management systems and new laws/regulations to better define incidence and prevalence of all types of hearing loss;

- Improved methods of data-collection, transmission, and data analytics positively impacting the ability to observe trends and modify diagnostic and intervention strategies accordingly; and

- Focused efforts to increase long-term data of children identified through EHDI programs offering insights into gaps in services, equitable access and efficacy.

\section{Tele-Practice}

- Provision of services through innovative methods such as telehealth resulting in more timely access and reduction of barriers that impact outcomes;

- Promotion of evidence-based policy changes needed to provide assurance of reimbursement for tele-practice services; and

- Expansion of tele-practice, as a constantly evolving mechanism for using technology to deliver a wide range of professional EHDI services.

Several issues need to be addressed before the benefits of tele-practice may be realized on a wide-scale basis.

These issues include the need for the following elements.

- Development of professional preparation and ongoing training to maintain expertise and familiarity with changes in technology and potential clinical applications (see ASHA practice portal on tele-practice); 
- Establishment of state licensure requirements and monitoring for tele-practice competencies for practitioners;

- Exploration of and advocacy for additional funding sources willing to reimburse providers for services rendered; and

- Identification of pre-requisite family skills needed to benefit from tele-practice delivery of services (e.g., able to navigate a computer, access technology, computer literate, etc.).

\section{Medical}

- Emergence of additional genetic discoveries and other medical breakthroughs to inform our understanding of congenital and late-onset or progressive hearing loss;

- Assurance of access to genetic testing for all families fostering an expansion in our ability to determine etiology while increasing our understanding of the underpinnings of congenital hearing loss;

- Recognition that while promising, the feasibility of wide scale implementation of gene-based therapies are unlikely in the near future due to heterogeneity of the etiology of hearing loss as well as cultural preferences;

- Consideration and ongoing investigation of screening for cCMV as a standard of care in all infants who do not pass their newborn hearing screen;

- Development of targeted interventions for children who are deaf or hard of hearing with additional disabilities in an effort to reduce the impact of comorbidities;

- Advances in hearing preservation surgery and cochlear implant candidacy have enormous potential for impacting the timing of this intervention;

- Reliable hearing preservation surgery will make cochlear implantation an earlier option for children following diagnosis; and

- Potential candidacy for early implant technology for unilateral/asymmetric hearing loss in select instances.

\section{Early Intervention}

In addition to this current 2019 position statement, early intervention professionals are encouraged to continue referring to the JCIH Supplement 2013. That document provided extensive coverage of best practice principles to guide interventions; only selected concepts are reinforced here.

- Increased timely support and guidance for families of newly identified infants to ensure that children have access to high quality language input (regardless of modalities or languages) from the earliest possible age;

- Provision of accurate and up-to-date information to families about all language and communication opportunities for their child, including visual, tactile, and listening technologies that support language learning;

- Participation in professional development and training to meet the changing nature and needs of families, preparing professionals with the knowledge and skills to provide services that are culturally and linguistically sensitive and responsive in all respects;

- Recognition of the increasing number of culturally and linguistically diverse children who are deaf or hard of hearing in the United States and its territories with targeted efforts to increase cultural competence;

- Intentional outreach and diversification of the workforce through both direct recruitment and increased access for underrepresented groups to professional preparation programs at universities so that families will have an increased likelihood of working with a professional of the same race or culture;

- Increased access to and inclusion of the Deaf Community as an important resource for families and providers in an effort to provide well-rounded services for families;

- Development of longitudinal research agendas to guide differentiation of practice and to expand evidence regarding specific interventions and intervention components and to inform family decision-making;

- Seamless access to and connections with family service coordinators with specialized knowledge of the needs of families and children who are deaf or hard of hearing, thereby decreasing time to service initiation;

- Development of strategies to connect families with resources and professionals providing adjustment counseling services (psychologists, counselors, infant mental health specialists) familiar with serving families of children who are deaf and hard of hearing;

- Increased progress toward integration of EHDI programs posited within state and territory agencies charged with implementation of Part B and Part C IDEA legislation;

- Collaboration with community evidence-based home visiting programs supported by rigorous research and recognized by the Maternal and Child Health Bureau to provide families with resources that may supplement early intervention programs;

- Broadening access to professionals with the skills, and knowledge, and disposition to deliver high quality early intervention services despite geographic, financial, and other barriers through technology (i.e., tele-practice).

\section{Research Needs}

- Continued and accelerated research into optimizing screening, diagnostic and amplification intervention protocols, emphasizing timeliness and accuracy based on rigorous evidence regarding efficacy;

- Exploration of pre-school hearing screening programs to determine the ability to identify late-onset or missed hearing loss;

- Increased longitudinal research on the efficacy and quality of early intervention strategies to assure optimal outcomes (developmental and quality of life) for children who are deaf or hard of hearing and their families;

- Increased inquiry and study of the cost utility/benefit of EHDI programs and systems;

- Focused research on the impact of social determinants of health outcomes relative to deafness or hearing loss; and 
- Further examination and delineation of health disparities due to geographic location (rural, inner-city), socio-economics, ethnic/racial groups and education.

\section{Acknowledgments}

The Year 2019 Position Statement was co-authored by the members of the Joint Committee on Infant Hearing $(\mathrm{JClH})$. The $\mathrm{JCIH}$ is funded by annual dues from each member organization to cover operational costs (e.g., member travel to meetings, sign language interpreters). All contributors conduct the work as volunteers on behalf of the organizations they represent and the $\mathrm{JCIH}$. They receive no remuneration and they have no conflicts of interest to declare. The views and opinions expressed in this position statement are those of the authors and do not necessarily reflect the offical policy or position of the member organizations.

$\mathrm{JClH}$ member organizations and their respective representatives who prepared this statement include (in alphabetical order): the Alexander Graham Bell Association for the Deaf and Hard of Hearing (Donald Goldberg, PhD, and Carianne Muse, MPH); the American Academy of Audiology (Alison M. Grimes, AuD, and Christine Yoshinaga-Itano, PhD); the American Academy of Otolaryngology-Head and Neck Surgery (Craig Buchman, MD, and Oliver Adunka, MD); the American Academy of Pediatrics (Rachel St. John, MD, and Betty Vohr, MD); the American Speech-

Language-Hearing Association (Patti Martin, PhD, and Ryan McCreery, PhD); the Council on Education of the Deaf, whose member organizations include the Alexander Graham Bell Association for the Deaf and Hard of Hearing, the American Society for Deaf Children, the Association of College Educators of the Deaf and Hard of Hearing, the Conference of Educational Administrators of Schools and Programs for the Deaf, the Convention of American Instructors of the Deaf, the National ASL and English Bilingual Consortium for Early Childhood Education, and the National Association of the Deaf (Jodee Crace, MA, and Stacy Abrams, MA), and the Directors of Speech and Hearing Programs in State Health and Welfare Agencies (Patricia Burk, MS; Kirsten Coverston, AuD; Beth Martin, $\mathrm{MA}$; and Alice Sette, AuD). Ex officio contributors to the $\mathrm{JCIH}$ include Pamela Mason, MEd, and Anne Oyler, AuD (American Speech-Language-Hearing Association). We also acknowledge the contribution of John Eichwald, MA, and Irene Forsman, MS, RN. Thank you to the organizations that provided additional support in numerous ways: Boys Town National Research Hospital, Health Resources and Services Administration, Maternal and Child Health Bureau, The Centers for Disease Control and Prevention, and The National Institute on Deafness and Other Communication Disorders, National Institutes of Health. We thank Nannette Nicholson, PhD, for numerous hours of editorial assistance and former $\mathrm{JCIH}$ members who contributed to the current document, including Beth S. Benedict, PhD [Chair 2012-2014]; Albert Mehl, MD; Cliff Megerian, MD; Mary Pat Moeller, PhD; and Judy Harrison, MA. We are grateful for their input, leadership, and assistance.

\section{References}

Acke, F. R., Dhooge, I. J., Malfait, F., \& De Leenheer, E. M. R. (2012). Hearing impairment in Stickler syndrome: A systematic review. Orphanet Journal of Rare Diseases, 7(1), 84.

https://doi.org/10.1186/1750-1172-7-84

Adebanjo, T., Godfred-Cato, S., Viens, L., Fischer, M., Staples, J. E., Kuhnert-Tallman, W., . . Moore, C. A. (2017). Update: Interim Guidance for the Diagnosis, Evaluation, and Management of Infants with Possible Congenital Zika Virus Infection-United States, October 2017. Morbidity and Mortality Weekly Report, 66 (41), 1089-1099.

https://doi.org/10.15585/mmwr.mm6641a1

Adunka, O. F., Jewells, V., \& Buchman, C. A. (2007). Value of computed tomography in the evaluation of children with cochlear nerve deficiency. Otology \& Neurotology, 28(5), 597-604.

https://doi.org/10.1097/01.mao.0000281804.36574.72

Agency for Healthcare Research and Quality (AHRQ). (n.d.). United States Health Information Knowledgebase (USHIK). Retrieved from https://ushik.ahrq.gov/mdr/portals

Alaerts, J., Luts, H., Van Dun, B., Desloovere, C., \& Wouters, J. (2010). Latencies of auditory steady-state responses recorded in early infancy. Audiology and Neurotology, 15(2), 116-127.

https://doi.org/10.1159/000231637

Alam, S. (2016). Progress in standardization of reporting and analysis of data from Early Hearing Detection and Intervention (EHDI) programs. Journal of Early Hearing Detection Intervention, 1(2), 2-7.

Alford, R. L., Arnos, K. S., Fox, M., Lin, J. W., Palmer, C. G., Pandya, A., ... Yoshinaga-Itano, C. (2014). American College of Medical Genetics and Genomics guideline for the clinical evaluation and etiologic diagnosis of hearing loss. Genetics in Medicine, 16(4), 347-355. https://doi.org/10.1038/gim.2014.2
Ambrose, S. E., Unflat Berry, L. M., Walker, E. A., Harrison, M., Oleson, J., \& Moeller, M. P. (2014). Speech sound production in 2-year-olds who are hard of hearing. American Journal of Speech Language Pathology, 23(2), 91-104. https://doi.org/10.1044/2014 AJSLP-13-0039

Ambrose, S. E., VanDam, M., \& Moeller, M. P. (2014). Linguistic input, electronic media, and communication outcomes of toddlers with hearing loss. Ear \& Hearing 35(2), 139-147.

https://doi.org/10.1097/AUD.0b013e3182a76768

American Academy of Audiology. (2012). Assessment of Hearing in Infants and Young Children. Reston, VA: Author. Retrieved from https://tinyurl.com/y3tbm2yy

American Academy of Audiology. (2013). American Academy of Audiology Clinical Practice Guidelines Pediatric Amplification. Retrieved from https://tinyurl.com/yysbaet8

American Academy of Audiology Subcommittee. (2011, September). American Academy of Audiology Childhood Hearing Screening Guidelines. Reston, VA. Retrieved from https://tinyurl.com/y6c5otur

American Academy of Pediatrics. (2014a). Guidelines for rescreening in the medical home following a "do not pass" newborn hearing screening. Retrieved from https://tinyurl.com/y5p2k953

American Academy of Pediatrics. (2014b). Reducing loss to follow-up/ document in newborn hearing screening: Guidelines for medical home providers. Retrieved from

http://www.aap.org/en-us/Documents/ehdi_Itfdguidelines.pdf

American Academy of Pediatrics Committee on Practice and Ambulatory Medicine and American Academy of Pediatrics Bright Futures Periodicity Schedule Workgroup. (2017). 2017 Recommendations for Preventive Pediatric Health Care. Pediatrics, 139(4), e20170254.

American Academy of Pediatrics Task Force for Improving Newborn 
Hearing Screening Diagnosis and Intervention. (2010). Early hearing detection and intervention (EHDI): Guidelines for pediatric medical home providers. Retrieved from https://tinyurl.com/y5zzowco

American Board of Audiology. (2016). Pediatric audiology specialty certification. Retrieved from http://www.boardofaudiology.org

American Speech-Language-Hearing Association (ASHA). (2008a). Guidelines for Audiologists Providing Informational and Adjustment Counseling to Families of Infants and Young Children with Hearing Loss Birth to 5 Years of Age. Rockville Pike, MD: Author.

https://doi.org/10.1044/policy.GL2008-00289

American Speech-Language-Hearing Association. (2008b). Loss to Follow-Up in Early Hearing Detection and Intervention (Technical Report). Rockville Pike, MD: Author.

https://doi.org/10.1044/policy.TR2008-00302

Arnold, C. L., Davis, T. C., Humiston, S. G., Bocchini, J. A., Jr, Bass, P. A., III, Bocchini, A., .. . Forsman, I. (2006). Infant hearing screening: Stakeholder recommendations for parent-centered communication. Pediatrics, 117(5, Pt. 2), S341-S354.

https://doi.org/10.1542/peds.2005-2633N

Åsberg, K. K., Vogel, J. J., \& Bowers, C. A. (2008). Exploring correlates and predictors of stress in parents of children who are deaf: Implications of perceived social support and mode of communication. Journal of Child and Family Studies, 17(4), 486-499.

https://doi.org/10.1007/s10826-007-9169-7

Atkinson, L., Paglia, A., Coolbear, J., Niccols, A., Parker, K. C. H., \& Guger, S. (2000). Attachment security: A meta-analysis of maternal mental health correlates. Clinical Psychology Review, 20(8), 1019-1040. https://doi.org/10.1016/S0272-7358(99)00023-9

Attias, J., \& Raveh, E. (2007). Transient deafness in young candidates for cochlear implants. Audiology and Neurootology, 12(5), 325-333.

Bagatto, M., Moodie, S., Brown, C., Malandrino, A., Richert, F., Clench, D., \& Scollie, S. (2016). Prescribing and verifying hearing aids applying the American Academy of Audiology Pediatric Amplification Guideline: Protocols and outcomes from the Ontario Infant Hearing Program. Journal of the American Academy of Audiology, 27(3), 188-203.

https://doi.org/10.3766/jaaa.15051

Barreira-Nielsen, C., Fitzpatrick, E., Hashem, S., Whittingham, J.,

Barrowman, N., \& Aglipay, M. (2016). Progressive hearing loss in early childhood. Ear \& Hearing, 37(5), e300-e321.

Behl, D. D., \& Kahn, G. (2015). Provider perspectives on telepractice for serving families of children who are deaf or hard of hearing. International Journal of Telerehabilitation, 7(1), 1-12. https://doi.org/10.5195/lJT.2015.6170

Berg, A. L., Prieve, B. A., Serpanos, Y. C., \& Wheaton, M. A. (2011). Hearing screening in a well-infant nursery: Profile of automated ABR-fail/ OAE-pass. Pediatrics, 127(2), 269-275. https://doi.org/10.1542/peds.2010-0676

Berg, A. L., Spitzer, J. B., Towers, H. M., Bartosiewicz, C., \& Diamond B. E. (2005). Newborn hearing screening in the NICU: Profile of failed auditory brainstem response/assed otoacoustic emission. Pediatrics, 116(4), 933-938. https://doi.org/10.1542/peds.2004-2806

Bergemalm, P. O. (2003). Progressive hearing loss after closed head injury: A predictable outcome? Acta Otolaryngology, 123(7), 836-845. https://doi.org/10.1080/00016480310002474

Berlin, C., Hood, L., Morlet, T., Wilensky, D., St. John, P., Montgomery, E., \& Thibodaux, M. (2005). Absent or elevated middle ear muscle reflexes in the presence of normal otoacoustic emissions: A universal finding in 136 cases of auditory neuropathy/dys-synchrony. Journal of the American Academy of Audiology, 16(8), 546-553.

Bhatia, P., Mintz, S., Hecht, B. F., Deavenport, A., \& Kuo, A. A. (2013). Early identification of young children with hearing loss in federally qualified health centers. Journal of Developmental \& Behavioral Pediatrics, 34(1), 15-21. https://doi.org/10.1097/DBP.0b013e318279899c

Biernath, K. R., Reefhuis, J., Whitney, C. G, Mann, E. A., Costa, P., Eichwald, J., \& Boyle, C. (2006). Bacterial meningitis among children with cochlear implants beyond 24 months after implantation. Pediatrics, 117(2), 284-289. https://doi.org/10.1542/peds.2005-0824

Blanco-Kelly, F., Jaijo, T., Aller, E., Avila-Fernandez, A., López-Molina, M. I., Giménez, A., . . . Ayuso, C. (2015). Clinical aspects of Usher syndrome and the USH2A gene in a cohort of 433 patients. JAMA Ophthalmology, 133(2), 157-164.

https://doi.org/10.1001/jamaophthalmol.2014.4498

Boone, R. T., Bower, C. M., \& Martin, P. F. (2005). Failed newborn hearing screens as presentation for otitis media with effusion in the newborn population. International Journal of Pediatric Otorhinolaryngology, 69(3), 393-397.
Buchman, C. A., Teagle, H. F. B., Roush, P. A., Park, L. R., Hatch, D., Woodard, J., . . Adunka, O. F. (2011). Cochlear implantation in children with labyrinthine anomalies and cochlear nerve deficiency: Implications for auditory brainstem implantation. Laryngoscope, 121(9), 1979-1988. https://doi.org/10.1002/lary.22032

Caesar, L. G., \& Kohler, P. D. (2007). The state of school-based bilingual assessment: Actual practice versus recommended guidelines. Language, Speech, and Hearing Services in Schools, 38(3), 190-200.

Campbell, J. D., Cardon, G., \& Sharma, A. (2011). Clinical application of the P1 cortical auditory evoked potential biomarker in children with sensorineural hearing loss and auditory neuropathy spectrum disorder. Seminars in Hearing, 32(2), 147-155

https://doi.org/10.1055/s-0031-1277236

Cannon, M. J., Griffiths, P. D., Aston, V., \& Rawlinson, W. D. (2014). Universal newborn screening for congenital CMV infection: What is the evidence of potential benefit? Reviews in Medical Virology, 24(5), 291-307. https://doi.org/10.1002/rmv.1790

Cardon, G., \& Sharma, A. (2013). Central auditory maturation and behavioral outcome in children with auditory neuropathy spectrum disorder who use cochlear implants. International Journal of Audiology, 17(Suppl 3), 82-99. https://doi.org/10.3109/14992027.2013.799786

Casey, K.-A., \& Small, S. A. (2014). Comparisons of auditory steady state response and behavioral air conduction and bone conduction thresholds for infants and adults with normal hearing. Ear \& Hearing, 35(4), 423-439. https://doi.org/10.1097/AUD.0000000000000021 Cebulla, M., \& Elberling, C. (2015). Auditory brain stem responses evoked by different chirps based on different delay models. Journal of the American Academy of Audiology, 21(7), 452-460.

https://doi.org/10.3766/jaaa.21.7.4

Cebulla, M., Lurz, H., \& Shehata-Dieler, W. (2014). Evaluation of waveform, latency and amplitude values of chirp ABR in newborns. International Journal of Pediatric Otorhinolaryngology, 78(4), 631-636. https://doi.org/10.1016/j.ijporl.2014.01.020

Centers for Disease Control and Prevention. (n.d.-a) Annual Data Early Hearing Detection and Intervention (EHDI) Program. Retrieved from http://www.cdc.gov/ncbddd/hearingloss/ehdi-data.html

Centers for Disease Control and Prevention. (n.d.-b). Early Hearing Detection and Intervention and Electronic Health Records Technology. Retrieved from http://www.cdc.gov/ncbddd/hearingloss/ehdi-hrt.html Centers for Disease Control and Prevention. (n.d.-c). Public Health Information Network Vocabulary Access and Distribution System (PHIN VADS). Retrieved from

https://phinvads.cdc.gov/vads/SearchVocab.action

Centers for Disease Control and Prevention. (2016a). Information about early hearing detection and intervention (EHDI) state programs.

Hearing loss in children. Retrieved from

http://www.cdc.gov/ncbddd/hearingloss/ehdi-programs.html

Centers for Disease Control and Prevention. (2016b). Pneumococcal vaccination. Vaccines and preventable diseases. Retrieved from http://www.cdc.gov/vaccines/vpd-vac/pneumo/

Centre for Allied Health Evidence Review Team. (2008). A Systematic Review of the Literature on Early Intervention for Children with a Permanent Hearing Loss: Vol II. Brisbane, Queensland: Joanna Briggs Institute. Retrieved from https://tinyurl.com/y35rzuc6

Ching, T. Y. C., \& Dillon, H. (2013). Major findings of the LOCHI study on children at 3 years of age and implications for audiological management. International Journal of Audiology, 52(2), S65-S68. https://doi.org/10.3109/14992027.2013.866339

Ching, T. Y. C., Dillon, H., Day, J., Crowe, K., Close, L., Chrisholm, K., \& Hopkins, T. (2009). Early language outcomes of children with cochlear implants: Interim findings of the NAL study on longitudinal outcomes of children with hearing impairment. Cochlear Implant International, 10

(Supp. 1), 28-32.

https://doi.org/10.1179/cim.2009.10.Supplement-1.28

Ching, T. Y. C., Dillon, H., Marnane, V., Hou, S., Day, J., Seeto, M., .

. Yeh, A. (2013). Outcomes of early- and late-identified children at 3 years of age: Findings from a prospective population-based study. Ear \& Hearing, 34(5), 535-552.

https://doi.org/10.1097/AUD.0b013e3182857718

Ching, T. Y. C., Leigh, G., \& Dillon, H. (2013). Introduction to the longitudinal outcomes of children with hearing impairment (LOCHI) study: Background, design, sample characteristics. International Journal of Audiology, 52(2), S4-S9.

https://doi.org/10.3109/14992027.2013.866342

Chu, C. W., Chen, Y. J., Lee, Y. H., Jaung, S. J., Lee, F. P., \& Huang, H. M. (2015). Government-funded universal newborn hearing 
screening and genetic analyses of deafness predisposing genes in Taiwan. International Journal of Pediatric Otorhinolaryngology, 79(4), 584-590. https://doi.org/10.1016/.i.jporl.2015.01.033

Chung, W., Beauchaine, K. L., Hoffman, J., Coverstone, K.R., Oyler, A., \& Mason, C. (2017). Early hearing detection and intervention-

Pediatric audiology links to services EHDI-PALS: Building a national facility database. Ear \& Hearing, 38(4), e227-e231. https://doi.org/10.1097/AUD.0000000000000426

Clark, R. H., Bloom, B. T., Spitzer, A. R., \& Gerstmann, D. R. (2006). Reported medication use in the neonatal intensive care unit: Data from a large national data set. Pediatrics, 117(6), 1979-1987. https://doi.org/10.1542/peds.2005-1707

Coenraad, S., Goedegebure, A., van Goudoever, J. B., \& Hoeve, L. J. (2010). Risk factors for sensorineural hearing loss in NICU infants compared to normal hearing NICU controls. International Journal of Pediatric Otorhinolaryngology, 74(9), 999-1002.

https://doi.org/10.1016/j.ijporl.2010.05.024

Colorado School for the Deaf and the Blind (CSDB) and Colorado Families for Hands \& Voices. (2011). Bridge to preschool: Navigating a successful transition. Retrieved from http://www.cohandsandvoices.org/docs/Bridge-Revised2012.pdf

Cone, B., \& Norrix, L. (2015). Measuring the advantage of KalmanWeighted Averaging for auditory brainstem response hearing evaluation in infants. American Journal of Audiology, 24(6), 153-168.

Cone, B., \& Whitaker, R. (2013). Dynamics of infant cortical auditory evoked potentials (CAEPs) for tone and speech tokens. International Journal of Otolaryngology, 77(7), 1162-1173.

https://doi.org/10.1016/j.ijporl.2013.04.030

Cone-Wesson, B., Vohr, B. R., Sininger, Y. S., Widen, J. E., Folsom, R. C., Gorga, M. P., \& Norton, S. J. (2000). Identification of neonatal hearing impairment: Infants with hearing loss. Ear and Hearing. 21(5), 488-507. https://doi.org/10.1097/00003446-200010000-00012

Council for Exceptional Children. (2014). DEC recommended practices in early intervention/early childhood special education 2014. Retrieved from http://www.dec-sped.org/recommendedpractices

Council on Community Pediatrics. (2009). The role of preschool home-visiting programs in improving children's developmental and health outcomes. Pediatrics, 123(2), 598-603.

https://doi.org/10.1542/peds.2008-3607

Crowe, K., McKinnon, D., McLeod, S., \& Ching, T. (2013). Multilingual children with hearing loss: Factors contributing to language use at home and in early education. Child Language Teaching and Therapy, 29(1), 111-129. https://doi.org/10.1177/0265659012467640

Crowe, K., Mcleod, S., \& Ching, T. Y. C. (2012). The cultural and linguistic diversity of 3-year-old children with hearing loss. Journal of Deaf Studies and Deaf Education, 17(4), 421-438.

https://doi.org/10.1093/deafed/ens028

Cruz, I., Quittner, A. L., Marker, C., \& DesJardin, J. L. (2013).

Identification of effective strategies to promote language in deaf children with cochlear implants. Child Development, 84(2), 543-559.

https://doi.org/10.1111/j.1467-8624.2012.01863.X

Dahl, H-H. M., Ching, T. Y. C., Hutchison, W., Hou, S., Seeto, M., \& Sjahalam-King, J. (2013). Etiology and audiological outcomes at 3 years for 364 children in Australia. PLoS One, 8(3), e59624.

https://doi.org/10.1371/journal.pone.0059624

Dammeyer, J. (2012). Development and characteristics of children with Usher syndrome and CHARGE syndrome. International Journal of Pediatric Otorhinolaryngology, 76(9), 1292-1296.

https://doi.org/10.1016/j.ijporl.2012.05.021

Daniell, W. E., Swan, S. S., McDaniel, M. M., Camp, J. E., Cohen, M. A., \& Stebbins, J. G. (2006). Noise exposure and hearing loss prevention programmes after 20 years of regulations in the United States. Occupational and Environmental Medicine, 63(5), 343-351.

Davis, T. C., Humiston, S. G., Arnold, C. L, Bocchini, J. A., Jr, Bass, P. F., III, Kennen, E. M., . . Lloyd-Puryear, M. (2006). Recommendations for effective newborn screening communication: Results of focus groups with parents, providers, and experts. Pediatrics, 177(5 Pt. 2),

S326-S340. https://doi.org/10.1542/peds.2005-2633M

Dedhia, K., Kitsko, D., Sabo, D., \& Chi, D. H. (2013). Children with sensorineural hearing loss after passing the newborn hearing screen. JAMA Otolaryngology-Head \& Neck Surgery, 139(2), 119-123. https://doi.org/10.1001/jamaoto.2013.1229

de Lyra-Silva, K. A., Sanches, S. G. G., Neves-Lobo, I. F., Ibidi, S. M., \& Carvallo, R. M. M. (2015). Middle ear muscle reflex measurement in neonates: Comparison between $1000 \mathrm{~Hz}$ and $226 \mathrm{~Hz}$ probe tones.
International Journal of Pediatric Otorhinolaryngology, 79(9),

1510-1515. https://doi.org/10.1016/j.ijporl.2015.06.039

DesGeorges, J. (2017). Family Support \& Cultural Competence. In L.

Schmelz (Ed.), EHDI E-Book (Chap. 14). Logan, UT: National Center for Hearing Assessment and Management. Retrieved from https://tinyurl.com/y2kt5hvt

DesJardin, J. L., Ambrose, S. E., \& Eisenberg, L. S. (2009). Literacy skills in children with cochlear implants: The importance of early oral language and joint storybook reading. Journal of Deaf Studies and Deaf Education, 14(1), 22-43. https://doi.org/10.1093/deafed/enn011.

DesJardin, J. L., \& Eisenberg, L. S. (2007). Maternal contributions: Supporting language development in young children with cochlear implants. Ear \& Hearing, 28(4):456-469.

https://doi.org/10.1097/AUD.0b013e31806dc1ab

Dettman, S. J., Pinder, D., Briggs, R. J. S., Dowell, R. C., \& Leigh, J. R. (2007). Communication development in children who receive the cochlear implant younger than 12 months: Risks versus benefits. Ear \& Hearing, 28(2 Suppl), 11S-18S.

https://doi.org/10.1097/AUD.0b013e31803153f8

Dillon, H., Cowan, R., \& Ching, T. Y. C. (2013). Longitudinal outcomes of children with hearing impairment (LOCHI). International Journal of Audiology, 52(Suppl. 2), S2-S3.

https://doi.org/10.3109/14992027.2013.866448

Dirks, E., Uilenburg, N., \& Rieffe, C. (2016). Parental stress among parents of toddlers with moderate hearing loss. Research in Developmental Disabilities, 55, 27-36. https://doi.org/10.1016/j.ridd.2016.03.008

Division for Early Childhood. (2015). Family Capacity-Building Practices Checklist. Retrieved from https://tinyurl.com/y2gpt9c8

Doutre, S. M., Barrett, T. S., Greenlee, J., \& White, K. R. (2016). Losing ground: Awareness of congenital Cytomegalovirus in the United States. Journal of Early Hearing Detection and Intervention, 1(2), 39-48.

Doyle, K. J., Kong, Y. Y., Strobel, K., Dallaire, P., \& Ray, R. M. (2004).

Neonatal middle ear effusion predicts chronic otitis media with effusion. Otology and Neurotology, 25(3), 318-322.

Dunst, C. J., \& Dempsey, I. (2007). Family-professional partnerships and parenting competence, confidence, and enjoyment. International Journal of Disability, Development and Education, 54(3), 305-318. https://doi.org/10.1080/10349120701488772

Dunst, C. J., Trivette, C. M., \& Deal, A. G. (1988). Enabling and Empowering Families: Principles and Guidelines for Practice. Cambridge, MA: Brookline Books.

Dunst, C., Trivette, C., \& Hamby, D. (2007). Meta-analysis of familycentered helpgiving practices research. Mental Retardation and Developmental Disabilities Research Reviews, 13(4), 370-378. https://doi.org/10.1002/mrdd.20176

Ealy, M., Lynch, K. A., Meyer, N. C., \& Smith, R. J. H. (2011). The prevalence of mitochondrial mutations associated with aminoglycoside-induced sensorineural hearing loss in an NICU population. Laryngoscope, 121(6), 1184-1186. https://doi.org/10.1002/lary.21778

Early Childhood Technical Assistance Center. (2016). Family-Centered Principles and Practices.

https://ectacenter.org/topics/eiservices/keyprinckeyprac.asp

Early Childhood Technical Assistance Center. (n.d.). Transition from Part $\mathrm{C}$ to preschool. Retrieved from

http://ectacenter.org/topics/transition/transition.asp

EHDI-PALS Advisory Group. (n.d.) Early hearing detection \&

intervention-Pediatric audiology links to services (EHDI-PALS).

Retrieved from http://www.ehdipals.org/

Eichwald J. (2016, March). Newborn hearing screening electronic clinical quality measure: EHDI eCQM. Presented at the Early Hearing Detection \& Intervention Meeting, San Diego, CA. Retrieved from https://tinyurl.com/y6804gte

Eisenberg, L. S., Widen, J. E., Yoshinaga-Itano, C., Norton, S., Thal, D., Niparko, J., \& Vohr, B. (2007). Current state of knowledge: Implications for developmental research-key issues. Ear \& Hearing, 28, 773-777. https://doi.org/10.1097/AUD.0b013e318157f06c

Eiserman, W. D., Hartel, D. M., Shisler, L., Buhrmann, J., White, K. R., \& Foust, T. (2008). Using otoacoustic emissions to screen for hearing loss in early childhood care settings. International Journal of Pediatric Otorhinolaryngology, 72(4), 475-482.

https://doi.org/10.1016/j.ijporl.2007.12.006

Eiserman, W. D., Shisler, L., Foust, T., Buhrmann, J., Winston, R., \& White, K. R. (2007). Screening for hearing loss in early childhood programs. Early Childhood Research Quarterly, 22(1), 105-117. https://doi.org/10.1016/j.ecresq.2006.09.001 
Ferm, I., Lightfoot, G., \& Stevens, J. (2013, Jan.) Comparison of ABR response amplitude, test time, and estimation of hearing threshold using frequency specific chirp and tone pip stimuli in newborns. International Journal of Audiology, 52, 419-423.

https://doi.org/10.3109/14992027.2013.769280

Filene, J. H., Kaminski, J. W., Valle, L. A., \& Cachat, P. (2013).

Components associated with home visiting program outcomes: A meta-analysis. Pediatrics, 132, S100-S109.

https://doi.org/10.1542/peds.2013-1021H

Fitzpatrick, E., Durieux-Smith, A., Eriks-Brophy, A., Olds, J., \& Gaines, R. (2007). The impact of newborn hearing screening on communication development. Journal of Medical Screening, 14(3), 123-131. https://doi.org/10.1258/096914107782066248

Fitzpatrick, E., Ham, J., \& Whittingham, J. (2015). Pediatric cochlear implantation: Why do children receive implants late? Ear \& Hearing, 36(6), 688-694. https://doi.org/10.1097/AUD.0000000000000184

Fleming-Dutra, K., Nelson, J., Fischer, M., Staples, J. E., Karwowski, M. P., Mead, P., . . Rasmussen, S. A. (2016). Update: Interim Guidelines for Health Care Providers Caring for Infants and Children with Possible Zika Virus Infection-United States, February 2016. Morbidity and Mortality Weekly Report, 65(7), 182-187. https://doi.org/10.15585/mmwr.mm6507e1

Fligor, B. J., Neault, M. W., Mullen, C. H., Feldman, H. A., \& Jones, D. T. (2005). Factors associated with sensorineural hearing loss among survivors of extracorporeal membrane oxygenation therapy. Pediatrics, 115(6), 1519-1528. https://doi.org/10.1542/peds.2004-0247

Fowler, K. B. (2013). Congenital cytomegalovirus infection: Audiologic outcome. Clinical Infectious Diseases, 57(Supp. 4), S182-S184. https://doi.org/10.1093/cid/cit609

Gaffney, M., Eichwald, J., Gaffney, C., \& Alam, S. (2014). Early hearing detection and intervention among infants-Hearing Screening and Follow-up Survey, United States, 2005-2006 and 2009-2010. Morbidity and Mortality Weekly Report, 62(2), 20-26. Retrieved from https://tinyurl.com/y5cfutaw

Gallaudet Research Institute. (2013, August). Regional and national summary report of data from the 2011-12 annual survey of deaf and hard of hearing children and youth. Washington, DC: GRI, Gallaudet University. Retrieved from https://tinyurl.com/yxdxq8mw

Gantz, B. J., Dunn, C. C., \& Walker, E. A. (2010). Bilateral cochlear implants in infants: A new approach--Nucleus Hybrid S12 project. Otology Neurotology, 31(8), 1300-1309.

https://doi.org/10.1097/MAO.0b013e3181f2eba1

Geers, A. E., Moog, J. S., Biedenstein, J., Brenner, C., \& Hayes, H. (2009). Spoken language scores of children using cochlear implants compared to hearing age-mates at school entry. Journal of Deaf Studies and Deaf Education, 14(3), 371-385.

https://doi.org/10.1093/deafed/enn046

Gerkin, L. A., \& Aslin, R. A. (2005). Thirty years of research on infant speech perception: The legacy of Peter W. Jusczyk. Language Learning and Development, 1(1), 5-21.

https://doi.org/10.1207/s15473341/ld0101_3

Gluth, M. B., Singh, R., \& Atlas, M. D. (2011). Prevention and management of cochlear implant infections. Cochlear Implants International, 12(4), 223-227.

https://doi.org/10.1179/146701011X12950038111576

Gorga, M. P., Johnson, T. A., Kaminski, J. R., Beauchaine, K. L., Garner, C. A., \& Neely, S. T. (2006). Using a combination of click- and tone burst-evoked auditory brain stem response measurements to estimate pure-tone thresholds. Ear \& Hearing, 27(1), 60-74. https://doi.org/10.1097/01.aud.0000194511.14740.9c

Gorga, M. P., Norton, S. J., Sininger, Y. S., Cone-Wesson, B., Folsom, R. C., Vohr, B. R., ... Neely, S. T. (2000). Identification of neonatal hearing impairment: Distortion product otoacoustic emissions during the perinatal period. Ear \& Hearing, 21(5), 400-424. Retrieved from http://www.ncbi.nlm.nih.gov/pubmed/11059701

Gracey, K. (2003). Current concepts in universal newborn hearing screening and early hearing detection and intervention programs. Advances in Neonatal Care, 3(6), 308-317. Retrieved from https://tinyurl.com/y67uyun4

Gravel, J., Berg, A., Bradley, M., Cacace, A., Campbell, D., Dalzell, L., . . Prieve, B. (2000). New York state universal newborn hearing screening demonstration project: Effects of screening protocol on inpatient outcome measures. Ear and Hearing, 21(2), 131-140.

Gruss, I., Handzel, O., Ingber, S., \& Beiser, M. (2012). Hearing loss due to chemotherapy and radiation therapy in young children. Harefuah, 151(1), 24-28.
Hall, J. W. (2016). Effective and efficient pre-school hearing screening: Essential for successful early hearing detection and intervention. Journal of American Academy of Audiology, 1(1), 2-12.

Hamilton, S., van Zuylen, W., Shand, A., Scott, G. M., Naing, Z., Hall, B., ... Rawlinson, W. D. (2014). Prevention of congenital cytomegalovirus complications by maternal and neonatal treatments: A systematic review. Reviews in Medical Virology. 24, 420-433.

https://doi.org/10.1002/rmv.1814

Hang, A. X., Roush, P. A., Teagle, H. F. B., Zdanski, C., Pillsbury, H., Adunka, O., \& Buchman, C. (2015). Is "no response" on diagnostic auditory brainstem response testing an indication for cochlear implantation in children? Ear \& Hearing, 36(1), 8-13. https://doi.org/10.1097/AUD.0000000000000072

Harrison, M., Page, T., Oleson, J., Spratford, M., Unflat Berry, L., Peterson, B., . . Moeller, M. P. (2016). Factors affecting early services for children who are hard of hearing. Language, Speech, and Hearing Services in Schools, 47, 16-30. Retrieved from https://tinyurl.com/y5zxvpe8

Hart, B., \& Risley, T. R. (1995). Meaningful Differences in the Everyday Experience of Young American Children. Baltimore, MD: Brookes Publishing Company.

He, S., Grose, J. H., Teagle, H. F. B., Woodard, J., Park, L., Hatch, D., \& Buchman, C. (2013). Gap detection measured with electrically evoked auditory event-related potentials and speech-perception abilities in children with auditory neuropathy spectrum disorder. Ear \& Hearing, 34(6), 733-744. https://doi.org/10.1097/AUD.0b013e3182944bb5 Hintermair, M. (2006). Parental resources, parental stress, and socioemotional development of deaf and hard of hearing children. Journal of Deaf Studies and Deaf Education, 11(4), 493-513. https://doi.org/10.1093/deafed/enl005

Hofmann, M., Luts, H., Poelmans, H., \& Wouters J. (2012). Investigation of a significant increase in referrals during neonatal hearing screening: $A$ comparison of Natus ALGO Portable and ALGO 3i. International Journal of Audiology, 51(1), 54-57. https://doi.org/10.3109/14992027.2011.625985

Hoffman, M. F., Quittner, A. L., \& Cejas, I. (2015). Comparisons of social competence in young children with and without hearing loss: A dynamic systems framework. Journal of Deaf Studies and Deaf Education, 20(2), 115-124. https://doi.org/10.1093/deafed/enu040

Hoffmann, A., Deuster, D., Rosslau, K., Knief, A., Am Zehnhoff-Dinnesen, A., \& Schmidt, C. M. (2013). Feasibility of $1000 \mathrm{~Hz}$ tympanometry in infants: Tympanometric trace classification and choice of probe tone in relation to age. International Journal of Pediatric Otorhinolaryngology, 77(7), 1198-1203. https://doi.org/10.1016/j.ijporl.2013.05.001

Holte, L., Walker, E., Oleson, J., Spratford, M., Moeller, M. P., Roush, P., ... Tomblin, J. B. (2012). Factors influencing follow-up to newborn hearing screening for infants who are hard of hearing. American Journal of Audiology, 21, 163-175.

https://doi.org/10.1044/1059-0889(2012/12-0016)

Hood, L. J. (2015). Auditory neuropathy/dys-synchrony disorder: Diagnosis and management. Otolaryngologic Clinics of North America, 48(6), 1027-1040. https://doi.org/10.1016/j.otc.2015.06.006

Hughes, M. L., Goehring, J. L., Baudhuin, J. L., Diaz, G. R., Sanford, T., Harpster, R., \& Valente, D. L. (2012). Use of telehealth for research and clinical measures in cochlear implant recipients: A validation study. Journal of Speech, Language, and Hearing Research, 55(4), 1112-1127. https://doi.org/10.1044/1092-4388(2011/11-0237)

Hunter, L. L., Prieve, B. A., Kei, J., \& Sanford, C. A. (2013). Pediatric applications of wideband acoustic immittance measures. Ear \& Hearing, 34(Supp. 1), 36S-42S. https://doi.org/10.1097/AUD.0b013e31829d5158

Hunter, L., Tubaugh, L., Jackson, A., \& Propes, S. (2008). Wideband middle ear power measurement in infants and children. Journal of the American Academy of Audiology, 19(4), 309-324.

Hurtado, N. (2009). Does input influence uptake? Links between maternal talk, processing speed and vocabulary size in Spanish-learning children. Developmental Science, 11(6).

https://doi.org/10.1111/j.1467-7687.2008.00768.x

Individuals with Disabilities Education Act Part C, 20 U.S.C. $\S 1400$ (2004).

Institute of Medicine. (2001). Crossing the Quality Chasm: A New Health System for the 21st Century. Washington D.C.: Institute of Medicine. https://doi.org/10.17226/10027

Iseli, C., \& Buchman, C. A. (2015). Management of children with severe, severe-profound, and profound sensorineural hearing loss.

Otolaryngology Clinics of North America, 48(6), 995-1010.

https://doi.org/10.1016/j.otc.2015.06.004 
Johnson, C. (2001). Supporting families in transition between early intervention and school age programs. Retrieved from

http://www.handsandvoices.org/pdf/trans_cheryl.pdf

Jackson, C. (2011). Family supports and resources for parents of children who are deaf or hard of hearing. American Annals of the Deaf, 254(4), 343-362.

Johnson, J. L., White, K. R., Widen, J. E., Gravel, J. S., James, M., Kennalley, T., . . . Holstrum, J. (2005a). A multicenter evaluation of how many infants with permanent hearing loss pass a two-stage otoacoustic emissions/automated auditory brainstem response newborn hearing screening protocol. Pediatrics, 116(3), 663-672.

https://doi.org/10.1542/peds.2004-1688

Johnson, J. L., White, K. R., Widen, J. E., Gravel, J. S., Vohr, B. R., James, M., . . .Meyer, S. (2005b). A multisite study to examine the efficacy of the otoacoustic emission/automated auditory brainstem response newborn hearing screening protocol: Introduction and over view of the study. American Journal of Audiology, 14(2), S178-S185. Retrieved from http://www.ncbi.nlm.nih.gov/pubmed/16489862 Johnson, R. F., Cohen, A. P., Guo, Y., Schibler, K., \& Greinwald, J. H. (2010). Genetic mutations and aminoglycoside-induced ototoxicity in neonates. Otolaryngology-Head \& Neck Surgery, 142 (5), 704-707. https://doi.org/10.1016/j.otohns.2010.01.030

Joint Commission. (n.d.). Joint Commission Oryx Performance Measurement System. Retrieved from

http://www.jointcommission.org/performance_measurement.aspx

Joint Committee on Infant Hearing. (2007). Year 2007 position statement Principles and guidelines for Early Hearing Detection and Intervention programs. Pediatrics, 120(4), 898-921.

https://doi.org/10.1542/peds.2007-2333

Joint Committee on Infant Hearing. (2013). Supplement to the JCIH 2007 position statement: Principles and guidelines for early intervention after confirmation that a child is deaf or hard of hearing. Pediatrics, 131(4), e1324-e1349. https://doi.org/10.1542/peds.2013-0008

Keefe, D. H., \& Simmons, J. L. (2003). Energy transmittance predicts conductive hearing loss in older children and adults. Journal of the Acoustic Society of America, 114(6), 3217-3238.

https://doi.org/10.1121/1.1625931

Kei, J. (2012). Acoustic stapedial reflexes in healthy neonates: Normative data and test-retest reliability. Journal of the American Academy of Audiology, 23(1), 46-56. https://doi.org/10.3766/jaaa.23.1.5

Kennedy, C. R., McCann, D. C., Campbell, M. J., Law, C. M., Mullee, M., Petrou, S., ... Stevenson, J. (2006). Language ability after early detection of permanent childhood hearing impairment. New England Journal of Medicine, 354(20), 2131-2141.

https://doi.org/10.1056/NEJMoa054915

Kim, S. H., Choi, H. S., Han, Y. E., \& Choi, B. Y. (2016). Diverse etiologies manifesting auditory neuropathy characteristics from infants with profound hearing loss and clinical implications. International Journal of Pediatric Otorhinolaryngology, 86, 63-67.

https://doi.org/10.1016/j.ijporl.2016.04.013

Kimberling, W. J., Hildebrand, M. S., Shearer, A. E., Jensen, M. L., Halder, J. A., Trzupek, K., . . Smith, R. J. H. (2010). Frequency of Usher syndrome in two pediatric populations: Implications for genetic screening of deaf and hard of hearing children. Genetics in Medicine, 12(8), 512-516. https://doi.org/10.1097/GIM.0b013e3181e5afb8

Korver, A., van Zanten, G., Meuwese-Jongejeugd, A., van Straaten, H., \& Oudesluys-Murphy, A. (2012). Auditory neuropathy in a low-risk population: A review of the literature. International Journal of Pediatric Otorhinolaryngology, 76(12), 1708-1711. https://doi.org/10.1016/j.ijporl.2012.08.009

Kuhn, D., \& Pease, M. (2006). Do children and adults learn differently? Journal of Cognition Development, 7(3), 279-293. https://doi.org/10.1207/s15327647jcd0703 1

Kushalnagar, P., Mathur, G., Mooreland, C., Napoli, D. J., Osterling, W., Padden, C., \& Rathmann, C. (2010). Infants and children with hearing loss need early language. Journal of Clinical Ethics, 21(2), 143-154. Retrieved from http://www.ncbi.nlm.nih.gov/pmc/articles/PMC3072291/ Lammens, F., Verhaert, N., \& Desloovere, C. (2013). Syndromic disorders in congenital hearing loss. B-ENT, 9(Supp. 21), 45-50.

Landry, S. H., Smith, K. E., \& Swank, P. R. (2006). Responsive parenting: Establishing early foundations for social, communication, and independent problem-solving skills. Developmental Psychology, 42(4), 627-642. https://doi.org/10.1037/0012-1649.42.4.627

Lasky, R. E., \& Williams, A. L. (2009). Noise and light exposures for extremely low birth weight newborns during their stay in the neonatal intensive care unit. Pediatrics, 123(2):540-546.

https://doi.org/10.1542/peds.2007-3418

Lasky, R. E., Wiorek, L., \& Becker, T. R. (1998). Hearing loss in survivors of neonatal extracorporeal membrane oxygenation (ECMO) therapy and high-frequency oscillatory (HFO) therapy. Journal of the American Academy of Audiology, 9(1), 47-58. Retrieved from https://tinyurl.com/y53limfg

Leal, M., Muniz, L., Caldas Neto, S. D., van der Linden, V., \& Ramos, R. (2016). Sensorineural hearing loss in a case of congenital Zika virus.

Brazilian Journal of Otorhinolaryngology.

https://doi.org/10.1016/j.bjorl.2016.06.001

Leal, M., Muniz, L., Ferreira, T., Santos, C. M., Almeida, L. C., . . Caldas Neto, S. D. (2016). Hearing Loss in Infants with Microcephaly and Evidence of Congenital Zika Virus Infection-Brazil, November 2015-May 2016. Morbidity and Mortality Weekly Report, 65(34), 917-919. https://doi.org/10.15585/mmwr.mm6534e3

Levit, Y., Himmelfarb, M., \& Dollberg, S. (2015). Sensitivity of the automated auditory brainstem response in neonatal hearing screening. Pediatrics, 136(3), e641-e647. https://doi.org/10.1542/peds.2014-3784 Lin, H., Shu, M., Lee, K., Lin, H., \& Lin, G. (2007). Reducing false positives in newborn hearing screening program: How and why comparison of referral rates after discharge. Otology \& Neurotology, 28, 788-792.

Lowe, L. H., \& Vézina, L. G. (2005). Sensorineural hearing loss in children. Radiographics, 17(5), 1079-1093.

https://doi.org/10.1016/S0140-6736(05)71047-3

Martini, A., Calzolari, F., \& Sensi, A. (2009). Genetic syndromes involving hearing. International Journal of Pediatric Otorhinolaryngology, 73, S2-S12. https://doi.org/10.1016/S0165-5876(09)70002-3

Mayne, A. M., Yoshinaga-Itano, C., Sedey, A. L., \& Carey, A. (1998). Expressive vocabulary development of infants and toddlers who are deaf or hard of hearing. Volta Review, 100(5), 1-28.

McCreery, R. W., Kaminski, J., Beauchaine, K., Lenzen, N., Simms, K., \& Gorga, M.P. (2014). The impact of degree of hearing loss on auditory brainstem response predictions of behavioral thresholds. Ear \& Hearing, 36(3), 309-319. https://doi.org/10.1097/AUD.0000000000000120 McCreery, R. W., Walker, E. A., Spratford, M., Bentler, R., Holte, L., Roush, P., . . Moeller, M. P. (2015). Longitudinal predictors of aided speech audibility in infants and children. Ear \& Hearing, 36(Supp. 1), 24S-37S.

McElveen, J. T., Blackburn, E. L., Green, J. D., McLear, P. W., Thimsen, D. J., \& Wilson, B. S. (2010). Remote programming of cochlear implants: A telecommunications model. Otology \& Neurotology, 31(7), 1035-1040. https://doi.org/10.1097/MAO.0b013e3181d35d87

Meadow-Orlans, K. (1994). Stress, support and deafness: Perceptions of infants' mothers and fathers. Journal of Early Intervention, 18, 91-102.

Mehra, S., Eavey, R. D., \& Keamy, D. G. (2009). The epidemiology of hearing impairment in the United States: Newborns, children, and adolescents. Otolaryngology-Head and Neck Surgery, 140(4), 461-472. Meinzen-Derr, J., Wiley, S., Creighton, J., \& Choo, D. (2007).

Auditory skills checklist: Clinical tool for monitoring functional auditory skill development in young children with cochlear implants. Annals of Otology, Rhinology, \& Laryngology, 116(11), 812-818.

Meinzen-Derr, J., Wiley, S., Grether, S., \& Choo, D. I. (2011). Children with cochlear implants and developmental disabilities: A language skills study with developmentally matched hearing peers. Research in Developmental Disabilities, 32(2), 757-767.

Melton, M. F., \& Backous, D. D. (2011). Preventing complications in pediatric cochlear implantation. Current Opinion in Otolaryngology \& Head and Neck Surgery, 19(5), 358-362. https://doi.org/10.1097/MOO.0b013e32834a023b

Morzaria, S., Westerberg, B. D., \& Kozak, F. K. (2005). Evidence-based algorithm for the evaluation of a child with bilateral sensorineural hearing loss. Journal of Otolaryngology, 34(5), 297-303.

Moeller, M. P., Carr, G., Seaver, L., Stredler-Brown, A., \& Holzinger D. (2013). Best practices in family-centered early intervention for children who are deaf or hard of hearing: An international consensus statement. The Journal of Deaf Studies and Deaf Education, 18(4), 429-445. https://doi.org/10.1093/deafed/ent034

Morris, B. H., Oh, W., Tyson, J. E., Stevenson, D. K., Phelps, D. L., O'Shea, T. M., ... Higgens, R. D. (2008). Aggressive vs. conservative phototherapy for infants with extremely low birth weight. New England Journal of Medicine, 359(18), 1885-1896.

https://doi.org/10.1056/NEJMoa0803024 
Morton, C. C., \& Nance, W. E. (2006). Newborn Hearing Screening-A Silent Revolution. New England Journal of Medicine, 354(20), 21512164. https://doi.org/10.1056/NEJMra050700

Nance, W. E. (2003). The genetics of deafness. Mental Retardation and Developmental Disabilities Research Reviews, 9(2), 109-119. https://doi.org/10.1002/mrdd.10067

Nance, W. E., Lim, B. G., \& Dodson, K. M. (2006). Importance of congenital cytomegalovirus infections as a cause for pre-lingual hearing loss. Journal of Clinical Virology, 35, 221-225.

https://doi.org/10.1016/j.jcv.2005.09.017

National Center for Hearing Assessment and Management. (n.d.). Newborn hearing screening training curriculum scripts. Retrieved from https://tinyurl.com/yyrywzxm

National Center for Hearing Assessment and Management. (2008). The Impact of Privacy Regulations: How EHDI, Part C, \& Health Providers Can Ensure That Children and Families Get Needed Services. Logan, UT: NCHAM. Retrieved from https://tinyurl.com/y629r3d9

National Center for Hearing Assessment and Management. (2013). Understanding the IDEA Part C Regulations: The Role of EHDI. Logan, UT: NCHAM.

National Institute for Children's Health Quality (NICHQ). (2016). Improving follow up for failed hearing screenings. Retrieved from https://tinyurl.com/y2yvjqqs

National Institute on Deafness and Other Communication Disorders. (2002). NIDCD Workshop on Congenital Cytomegalovirus Infection and Hearing Loss, Rockville, MD. Retrieved from https://tinyurl.com/y6k8tbvs National Institute on Deafness and Other Communication Disorders. (2005). NIDCD statistical report; Prevalence of hearing loss in U.S. children, 2005. Rockville, MD: Author. Retrieved from https://tinyurl.com/y6k8tbvs

National Institute of Health. (1993). Early Identification of Hearing Impairment in Infants and Young Children. NIH Consensus Development Conference Statement. Bethesda, MD.

National Quality Forum. (n.d.-a). Audiological evaluation no later than 3 months of age. NFQ \#1360. Retrieved from

http://www.qualityforum.org/Qps/QpsTool.aspx

National Quality Forum. (n.d.-b). Hearing Screening Prior To Hospital Discharge (\#1354). Retrieved from https://tinyurl.com/y5b9c5fc

National Quality Forum. (n.d.-c). Intervention no later than 6 months of age. NQF \#1361. Retrieved from

http://www.qualityforum.org/Qps/QpsTool.aspx

National Quality Forum. (n.d.-d). Measures, Report \& Tools. Retrieved from http://www.qualityforum.org/Measures Reports Tools.aspx

Nelson, H. D., Bougatsos, C., \& Nygren, P. (2008). Universal newborn hearing screening: Systematic review to update the 2001 U.S. preventive services task force recommendation. Pediatrics, 122(1), e266-e276. https://doi.org/10.1542/peds.2007-1422

Nicholas, J. G., \& Geers, A. E. (2007). Will they catch up? The role of age at cochlear implantation in the spoken language development of children with severe to profound hearing loss. Journal of Speech, Language, and Hearing Research, 50(4), 1048-1062. https://doi.org/10.1044/1092-4388(2007/073)

Nicholson, N., Atcherson, S. R., Martin, P., Spragins, M. G., Schlagenhauf, L., \& Zraick, R. I. (2016). Readability, user-friendliness, and key content analysis of newborn hearing screening brochures. Journal of Early Hearing Detection and Intervention, 1, 66-77. https://doi.org/10.15142/T36C7N

Nicholson, N., Christensen, L., Dornhoffer, J., Martin, P., \& Smith-Olinde, L. (2011). Verification of speech spectrum audibility for pediatric Baha Softband users with craniofacial anomalies. Cleft Palate-Craniofacial Journal, 48(1), 55-65. https://doi.org/10.1597/08-17

Niparko, J. K., Tobey, E. A., Thal, D. J., Eisenberg, L. S., Wang, N., Quittner, A. L., \& Fink, N. E. (2010). Spoken language development in children following cochlear implantation. JAMA, 303(15), 1498-1506. https://doi.org/10.1001/jama.2010.451

Niskar, A. S., Kieszak, S. M., Holmes, A., Esteban, E., Rubin, C., \& Brody, D. J. (1998, Apr. 8). Prevalence of hearing loss among children 6 to 19 years of age: The third national health and nutrition examination survey. Journal of the American Medical Association, 279(14), 1071-1075.

Norrix, LW. (2015). Hearing thresholds, minimum response levels, and cross-check measures in pediatric audiology. American Journal of Audiology, 24(2), 137-144. https://doi.org/10.1044/2015_AJA-14-0095

Norton, S. J., Gorga, M. P., Widen, J. E., Folsom, R. C., Sininger, Y., Cone-Wesson, B., . . . Fletcher, K. (2000a). Identification of neonatal hearing impairment: Evaluation of transient evoked otoacoustic emission, distortion product otoacoustic emission, and auditory brain stem response test performance. Ear \& Hearing, 21(5), 508-528. https://doi.org/10.1097/00003446-200010000-00013

Norton, S. J., Gorga, M. P., Widen, J. E., Folsom, R., Sininger, Y., Cone-Wesson, B., . . . Fletcher, K. (2000b). Identification of neonatal hearing impairment: Summary and recommendations. Ear and Hearing, (21), 529-535. https://doi.org/10.1097/00003446-200010000-00014

Oberg, C., Colianni, S., \& King-Schultz, L. (2016). Child health disparities in the 21st Century. Current Problems in Pediatric and Adolescent Health Care, 46(9), 291-312.

Oh, W., Tyson, J. E., Fanaroff, A. A., Vohr, B. R., Perritt, R, Stoll, B. J., . . Wright, L. L. (2003). Association between peak serum bilirubin and neurodevelopmental outcomes in extremely low birth weight infants. Pediatrics, 112(4), 773-779. https://doi.org/10.1542/peds.112.4.773 Olusanya, B. O. (2011). Highlights of the new WHO report on newborn and infant hearing screening and implications for developing countries. International Journal of Pediatric Otorhinolaryngology, 75(6), 745-748. https://doi.org/10.1016/j.ijporl.2011.01.036

Orzan, E., \& Murgia, A. (2007). Connexin 26 deafness is not always congenital. International Journal of Pediatric Otorhinolaryngology, 71(3), 501-507. https://doi.org/10.1016/j.ijporl.2006.12.002

Parner, E. T., Reefhuis, J., Schendel, D., Thomsen, J. L., Ovesen, T., \& Thorsen, P. (2007). Hearing loss diagnosis followed by meningitis in Danish children, 1995-2004. Otolaryngology-Head Neck Surgery, 136(3), 428-433. https://doi.org/10.1016/j.otohns.2006.10.008

Pipp-Siegel, S., Sedey, A. L., Van Leeuwen, A. M., \& Yoshinaga-Itano, C. (2003). Mastery motivation and expressive language in young children with hearing loss. Journal of Deaf Studies and Deaf Education, 8(2), 133-145.

Pipp-Siegel, S., Sedey, A. L., \& Yoshinaga-Itano, C. (2002). Predictors of parental stress in mothers of young children with hearing loss. Journal of Deaf Studies and Deaf Education, 7(1), 1-17.

https://doi.org/10.1093/deafed/7.1.1

Pizur-Barnekow, K., Darragh, A., \& Johnston, M. (2011). "I cried because I didn't know if I could take care of him": Toward a taxonomy of interactive and critical health literacy as portrayed by caregivers of children with special health care needs. Journal of Health

Communication, 16(Supp. 3), 205-221.

https://doi.org/10.1080/10810730.2011.604386

Poliva, O. (2016). From Mimicry to Language: A neuroanatomically based evolutionary model of the emergence of vocal language. Frontiers in Neuroscience, 10, 307. https://doi.org/10.3389/fnins.2016.00307

Prieve, B. A., Beauchaine, K. L., Sabo, D., Schooling, T., Culpepper, B., \& Tharpe, A. M. (2013). Evidence-based systematic review of newborn hearing screening using behavioral audiometric threshold as a gold standard. Rockville Pike: ASHA. Retrieved from https://tinyurl.com/y6foxls7

Prieve, B. A., Schooling, T., Venediktov, R., \& Franceschini, N. (2015). An evidence-based systematic review on the diagnostic accuracy of hearing screening instruments for preschool and school-age children. American Journal of Audiology, 24(2), 250-267. https://doi.org/10.1044/2015 AJA-14-0065

Prieve, B. A., Vander Werff, K. R., Preston, J. L., \& Georgantas, L. (2013). Identification of conductive hearing loss in young infants using tympanometry and wideband reflectance. Ear \& Hearing, 34(2), 168-178.

Prosser, J. D., Cohen, A. P., \& Greinwald, J. H. (2015). Diagnostic evaluation of children with sensorineural hearing loss. Otolaryngologic Clinics of North America, 48(6), 975-982.

https://doi.org/10.1016/j.otc.2015.07.004

Punch, S., Van Dun, B., King, A., Carter, L., \& Pearce, W. (2016). Clinical experience of using cortical auditory evoked potentials in the treatment of infant hearing loss in Australia. Seminars in Hearing, 37(1), 36-52.

Quittner, A. L., Cruz, I., Barker, D. H., Tobey, E., Eisenberg, L. S., \& Niparko, J. K. (2013). Effects of maternal sensitivity and cognitive and linguistic stimulation on cochlear implant users' language development over four years. Journal of Pediatrics, 162(2), 343-348.

https://doi.org/10.1016/j.jpeds.2012.08.003

Rajenderkumar, D., Bamiou, D., \& Sirimanna, T. (2005). Management of hearing loss in Apert syndrome. Journal of Laryngology Otology, 119(5), 385-390. https://doi.org/10.1258/0022215053945714

Robertson, C. M. (1995). Hearing loss among children who have under gone ECMO. Canadian Medical Association Journal, 153(7), 881.

Robertson, C. M. T., Howarth, T. M., Bork, D. L. R., \& Dinu, I. A. (2009). Permanent bilateral sensory and neural hearing loss of children after 
neonatal intensive care because of extreme prematurity: A thirty-year study. Pediatrics, 123(5), e797-e807.

https://doi.org/10.1542/peds.2008-2531

Roche, J. P., Huang, B. Y., Castillo, M., Bassim, M. K., Adunka, O. F., \& Buchman, C. A. (2010). Imaging characteristics of children with auditory neuropathy spectrum disorder. Otology \& Neurotology, 31(5), 780-788. Retrieved from https://tinyurl.com/y6r4wjjq

Roizen, N. J., Magyar, C. I., Kuschner, E. S., Sulkes, S. B., Druschel, C., van Wijngaarden, E., ... Hyman, S. L. (2014). A community cross-sectional survey of medical problems in 440 children with down syndrome in New York state. Journal of Pediatrics 164(4), 871-875. https://doi.org/10.1016/i.jpeds.2013.11.032

Rosenfeld, R. M., Schwartz, S. R., Pynnonen, M. A., Tunkel, D. E., Hussey, H. M., Fichera, J. S., . . . Schellhase, K. G. (2013). Clinical practice guideline: Tympanostomy tubes in children-Executive summary. Otolaryngology Head Neck Surgery, 149(1), 8-16.

Retrieved from: http://www.ncbi.nlm.nih.gov/pubmed/23818537

Ross, S. A., \& Ahmed, A., Palmer, A. L., (2014). Detection of congenital cytomegalovirus infection by real-time polymerase chain reaction analysis of saliva or urine specimens. Journal of Infectious Diseases, 210(9), 1415-1418. https://doi.org/10.1093/infdis/jiu263

Rubin, L. G., \& Papsin, B. (2010). Cochlear implants in children: Surgical site infections and prevention and treatment of acute otitis media and meningitis. Pediatrics, 126(2), 381-391.

https://doi.org/10.1542/peds.2010-1427

Rush, D., \& Shelden, M. L. L. (2011). The Early Childhood Coaching Handbook. Baltimore, MD: Brookes Publishing Co.

Russell, K., Oliver, S., Lewis, L., Barfield, W. D., Cragan, J.,

Meaney-Delman, D., . . . Rasmussen, S. A. (2016). Update: Interim guidance for the evaluation and management of infants with possible congenital zika virus infection-United States, August 2016. Morbidity and Mortality Weekly Report, 65(33), 870-878.

https://doi.org/10.15585/mmwr.mm6533e2

Rutherford, K. D., Lerer, T. S., Schoem, S. R., \& Valdez, T. A. (2011). Evaluation of pediatric sensorineural hearing loss: A survey of pediatric otolaryngologists. Annals of Otology, Rhinology, \& Laryngology, 120(10), 674-681.

Santos, R. L. P., Aulchenko, Y. S., Huygen, P. L. M., van der Donk, K. P., de Wijs, I. J., Kemperman, M. H., . . Cremers C. (2005). Hearing impairment in Dutch patients with connexin 26 (GJB2) and connexin 30 (GJB6) mutations. International Journal of Pediatric Otorhinolaryngology, 69(2), 165-174. https://doi.org/10.1016/j.ijporl.2004.08.015

Scherf, F. W. A. C., van Deun, L., van Wieringen, A., Wouters, J., Desloovere, C., Dhooge, I., ... Van de Heyning, P. H. (2009a). Functional outcome of sequential bilateral cochlear implantation in young children: 36 months postoperative results. International Journal of Pediatric Otorhinolaryngology, 73(5), 723-730. https://doi.org/10.1016/j.ijporl.2009.01.009

Scherf, F., Van Deun, L., van Wieringen, A., Wouters, J., Desloovere, C., Dhooge, I., . . Van de Heyning, P. (2009b). Three-year postimplantation auditory outcomes in children with sequential bilateral cochlear implantation. Annals of Otology, Rhinology, \& Laryngology, 118(5), 336-344.

Seewald, R., Moodie, S., Scollie, S., \& Bagatto, M. (2005). The DSL method for pediatric hearing instrument fitting: Historical perspective and current issues. Trends in Hearing, 9, 145-157. https://doi.org/10.1177/108471380500900402

Shankaran, S., Pappas, A., McDonald, S. A., Vohr, B. R., Hintz, S. R., Yolton, K., . . Higgins, R. D. (2012). Childhood outcomes after hypothermia for neonatal encephalopathy. New England Journal of Medicine, 366(22), 2085-2092. https://doi.org/10.1056/NEJMoa1112066

Shapiro, S. M. (2003). Bilirubin toxicity in the developing nervous system. Pediatric Neurology, 29(5), 410-421.

https://doi.org/10.1016/j.pediatrneurol.2003.09.011

Sharma, A., Cardon, G., Henion, K., \& Roland, P. (2011). Cortical maturation and behavioral outcomes in children with auditory neuropathy spectrum disorder. International Journal of Audiology, 50(2), 98-106. https://doi.org/10.3109/14992027.2010.542492

Sininger, Y. S., Grimes, A., \& Christensen, E. (2010). Auditory development in early amplified children: Factors influencing auditory-based communication outcomes in children with hearing loss. Ear \& Hearing, 31(2), 166-185. https://doi.org/10.1097/AUD.0b013e3181c8e7b6

Small, A., Ishida, I., \& Stapells, D. (2017). Infant cortical auditory evoked potentials to lateralized noise shifts produced by changes in interaural time difference. Ear \& Hearing, 38(1), 94-102.
Smets, K., De Coen, K., Dhooge, I., Standaert, L., Laroche, S., Mahieu, L., . . Boudewyns, A. (2006). Selecting neonates with congenital cytomegalovirus infection for ganciclovir therapy. European Journal of Pediatrics, 165(12), 885-890.

https://doi.org/10.1007/s00431-006-0192-2

Sousa, A. C., Didoné, D. D., \& Sleifer, P. (2016). Longitudinal comparison of auditory steady-state evoked potentials in preterm and term infants:

The maturation process. International Archives of Otorhinolaryngology, 21(3), 200-205. https://doi.org/10.1055/s-0036-1584888

Staples, J., Dziuban, E., Fischer, M, Cragan, J. D., Rasmussen, S. A., Cannon, M. J., . . Moore, C. A. (2016). Interim guidelines for the evaluation and testing of infants with possible congenital zika virus infection-United States, 2016. Morbidity and Mortality Weekly Report, 65(3), 63-67. https://doi.org/10.15585/mmwr.mm6503e3

Starr, A., Picton, T. W., Sininger, Y. Y., Hood, L. J., \& Berlin, C. I. (1996). Auditory neuropathy. Brain, 119(Pt. 3), 741-753.

Steel, M. M., Papsin, B. C., \& Gordon, K. A. (2015). Binaural fusion and listening effort in children who use bilateral cochlear implants: A psychoacoustic and pupillometric study. PLoS One, 10(2), 1-29. https://doi.org/10.1371/journal.pone.0117611

Stika, C. J., Eisenberg, L. S., Johnson, K. C., Henning, S. C., Colson, B. G., Ganguly, D. H., \& DesJardin, J. L. (2015). Developmental outcomes of early-identified children who are hard of hearing at 12 to 18 months of age. Early Human Development, 91(1), 47-55.

https://doi.org/10.1016/j.earlhumdev.2014.11.005

Stuart, A., \& Cobb, K. M. (2014). Effect of stimulus and number of sweeps on the neonate auditory brainstem response. Ear \& Hearing, 35(5), 585-588. https://doi.org/10.1097/AUD.0000000000000066

Sun, L., Li, G., Miller, T., Salorio, C., Byrne, M. W., Bellinger, D. C., . . . McGowan, F. X. (2016). Association between a single general anesthesia exposure before age 36 months and neurocognitive outcomes in later childhood. JAMA, 315(21), 2312-2320.

Suskind, D., Leffel, K. R., Hernandez, M. W., Sapolich, S. G., Suskind, E., Kirkham, E., \& Meehan, P. (2013). An Exploratory Study of "Quantitative Linguistic Feedback": Effect of LENA Feedback on Adult Language Production. Communication Disorders Quarterly, 34(4), 199-209. https://doi.org/10.1177/1525740112473146

Swanepoel, D. W., \& Hall, J. W. (2010). A systematic review of tele health applications in audiology. Telemedicine Journal, 16(2), 181-200. https://doi.org/10.1089/tmj.2009.0111

Szagun, G., \& Schramm, S. (2016). Sources of variability in language development of children with cochlear implants: Age at implantation, parental language, and early features of children's language construction. Journal of Child Language, 43(3), 505-536.

https://doi.org/10.1017/S0305000915000641

Tarkan, O., Sari, P., Demirhan, O., (2013). Connexin 26 and 30 mutations in paediatric patients with congenital, non-syndromic hearing loss treated with cochlear implantation in Mediterranean Turkey. Journal of Laryngology \& Otology, 127(1), 33-37.

https://doi.org/10.1017/S0022215112002587

Tomblin, J. B., Harrison, M., Ambrose, S. E., Walker, E. A., Oleson, J. J., \& Moeller, M. P. (2015). Language outcomes in young children with mild to severe hearing loss. Ear and Hearing, 36, 76S-96S.

https://doi.org/10.1097/AUD.0000000000000219

Tomblin, J. B., Oleson, J. J., Ambrose, S. E., Walker, E., \& Moeller, M. P. (2014). The influence of hearing aids on the speech and language development of children with hearing loss. JAMA Otolaryngoly Head and Neck Surgery. 140(5), 403-409.

https://doi.org/10.1001/jamaoto.2014.267

Topol, D., Girard, N., St. Pierre, L., Tucker, R., \& Vohr, B. (2011). The effects of maternal stress and child language ability on behavioral outcomes of children with congenital hearing loss at 18-24 months. Early Human Development, 87(12), 807-811.

https://doi.org/10.1016/j.earlhumdev.2011.06.006

Uhler, K., Heringer, A., Thompson, N., \& Yoshinaga-Itano, C. (2012). A tutorial on auditory neuropathy/dyssynchrony for the speech-language pathologist and audiologist. Seminars in Speech and Language, 33(4), 354-366. https://doi.org/10.1055/s-0032-1326917

Uhler, K., Thomson, V., Cyr, N., Gabbard, S. A., \& Yoshinaga-Itano, C. (2014). State and territory EHDI databases: What we do and don't know about the hearing or audiological data from identified children. American Journal of Audiology, 23(1), 34-43.

https://doi.org/10.1044/1059-0889(2013/13-0015)

U.S. Department of Education. (n.d.-a). Building the Legacy: IDEA 2004. Retrieved from

https://sites.ed.gov/idea/search-wpsolr/?wpsolr_q=building+the+legacy 
U.S. Department of Education. (n.d.-b). Family Educational Rights and Privacy Act (FERPA). Retrieved from https://ed.gov/policy/gen/guid/fpco/ferpa/index.html

U.S. Department of Health and Human Services. (2017). Health Information Privacy and Portability. Washington, DC: Author. Retrieved from http://www.hhs.gov/hipaa/index.html

U.S. National Library of Medicine. (n.d.-a). Newborn Screening Coding and Terminology Guide. Retrieved from https://newbornscreeningcodes.n/m.nih.gov/

U.S. National Library of Medicine. (n.d.-b). Value Set Authority Center. Retrieved from https://vsac.nlm.nih.gov

Van Camp, G., \& Smith, R. (2017). Hereditary Hearing Loss Homepage. Retrieved from http://hereditaryhearingloss.org/

Vander Werff, K. R., Prieve, B. A., \& Georgantas, L. M. (2007). Test-retest reliability of wideband reflectance measures in infants under screening and diagnostic test conditions. Ear \& Hearing, 28, 669-681.

https://doi.org/10.1097/AUD.0b013e31812f71b1

Van Dyk, M., Swanepoel, D. W., Hall, J. W. (2015). Outcomes with OAE and AABR screening in the first $48 \mathrm{~h}-$ Implications for newborn hearing screening in developing countries. International Journal of Pediatric Otorhinolaryngology, 79(7), 1034-1040.

https://doi.org/10.1016/j.ijporl.2015.04.021

Van Maanen, A., \& Stapells, D. R. (2010). Multiple-ASSR thresholds in infants and young children with hearing loss. Journal of the American Academy of Audiology, 21(8), 535-545.

https://doi.org/10.3766/jaaa.21.8.5

Vohr, B., Topol, D., Girard, N., St. Pierre, L., Watson, V., \& Tucker, R. (2012). Language outcomes and service provision of preschool children with congenital hearing loss. Early Human Development, 88(7), 493-498. https://doi.org/10.1016/j.earlhumdev.2011.12.007

Voss, S. E., Herrmann, B. S., Horton, N. J., Amadei, E. A., \& Kujawa, S. G. (2016). Reflectance measures from infant ears with normal hearing and transient conductive hearing loss. Ear \& Hearing, 37(5), 560-571. https://doi.org/10.1097/AUD.0000000000000293

Walker, E. A., Holte, L., McCreery, R. W., Spratford, M., Page, T., \& Moeller, M. P. (2015). The influence of hearing aid use on outcomes of children with mild hearing loss. Journal of Speech, Language, and Hearing Research. 58(5), 1611-1625.

Walker, E. A., Holte, L., Spratford, M., Oleson, J., Welhaven, A., \& Harrison, M. (2014). Timeliness of service delivery for children with later-identified mild-to-severe hearing loss. American Journal of Audiology, 23(1), 116-128. https://doi.org/10.1044/1059-0889(2013/13-0031)

Watkin, P., \& Baldwin, M. (2012). The longitudinal follow up of a universal neonatal hearing screen: The implications for confirming deafness in childhood. International Journal of Audiology, 51(7), 519-528. https://doi.org/10.3109/14992027.2012.673237

Watkin, P., McCann, D., Law, C., Mullee, M., Petrou, S., Stevenson, J., Kennedy, C. (2007). Language ability in children with permanent hearing impairment: The influence of early management and family participation. Pediatrics, 120(3), e694-e701.

https://doi.org/10.1542/peds.2006-2116

Watkins, S., Pittman, P., \& Walden, B. (1998). The deaf mentor experimental project for young children who are deaf and their families. American Annals of the Deaf, 143(1), 29-34.

https://doi.org/10.1353/aad.2012.0098

Weisel, A., Most, T., \& Michael, R. (2007). Mothers' stress and expectations as a function of time since child's cochlear implantation. Journal of Deaf Studies and Deaf Education, 12(1), 55-64. https://doi.org/10.1093/deafed/enl020

White, K. R., Nelson, L. H., \& Munoz, K. F. (2016). How many babies with hearing loss will be missed by repeated newborn hearing screening with otoacoustic emissions due to statistical artifact? Journal of Early Hearing Detection and Intervention, 1(2), 56-62.

Whitton, J. P., \& Polley, D. B. (2011). Evaluating the perceptual and pathophysiological consequences of auditory deprivation in early postnatal life: A comparison of basic and clinical studies. Journal of the Association for Research in Otolaryngology, 12(5), 535-546.

https://doi.org/10.1007/s10162-011-0271-6
Wickremasinghe, A. C., Risley, R. J., Kuzniewicz, M. W., Wu, Y. W., Walsh, E. M., Wi, S., McCullloch, C. E., \& Newman, T. B. (2015). Risk of sensorineural hearing loss and bilirubin exchange transfusion thresholds. Pediatrics, 136(3), 505-512.

https://doi.org/10.1542/peds.2014-3357

Widen, J., Johnson, J. L., White, K. R., Gravel, J. S., Vohr, B. R., James, M., ... Meyer, S. (2005). A multisite study to examine the efficacy of the otoacoustic emission/automated auditory brainstem response newborn hearing screening protocol: Results of visual reinforcement audiometry. American Journal of Audiology, 14, S2000-S2216.

Widen, J. E., \& Keener, S. K. (2003). Diagnostic testing for hearing loss in infants and young children. Mental Retardation and Developmental Disabilities Research and Review, 9(4):220-224.

https://doi.org/10.1002/mrdd.10083

Wiley, S., \& Meinzen-Derr, J. (2013). Use of the ages and stages questionnaire in young children who are deaf/hard of hearing as a screening for additional disabilities. Early Human Development, 89(10), 294-300.

Williams, T. R., Alam, S., \& Gaffney, M. (2015). Progress in identifying infants with hearing loss-United States, 2006-2012. Morbid Mortality Weekly Report, 64(13), 351-355.

Winston-Gerson, R., \& Hoffman, J. (2017). Tracking Reporting \& Follow-Up. In L. Schmelz (Ed.), EHDI E-Book (Chap. 3). Logan, UT: National Center for Hearing Assessment and Management. Retrieved from https://tinyurl.com/y4y73wk2

Wood, S. A., Davis, A. C., \& Sutton, G. J. (2013). Effectiveness of targeted surveillance to identify moderate to profound permanent childhood hearing impairment in babies with risk factors who pass newborn screening. International Journal of Audiology, 52(6), 394-399. https://doi.org/10.3109/14992027.2013.769067

Vohr, B. R., Widen, J. E., Cone-Wesson, B., Sininger, Y. S., Gorga, M. P., Folsom, R., \& Norton, S. J. (2000). Identification of neonatal hearing impairment: Characteristics of infants in the neonatal intensive care unit and well-baby nursery. Ear \& Hearing, 21, 373-382. https://doi.org/10.1097/00003446-200010000-00005

Yamamoto, A. Y., Mussi-Pinhata, M. M., Isaac, M. L., Amaral, F. R., Carvalheiro, C. G., Aragon, D. C., . . Britt, W. J. (2011). Congenital cytomegalovirus infection as a cause of sensorineural hearing loss in a highly immune population. The Pediatric Infectious Disease Journal, 30(12), 1043-1046. Retrieved from https://tinyurl.com/y2mh9q8h Yoshinaga-Itano, C., Baca, R. L., \& Sedey, A. L. (2010). Describing the trajectory of language development in the presence of severe-to-profound hearing loss: A closer look at children with cochlear implants versus hearing aids. Otology \& Neurotology, 31(8), 1268-1274. https://doi.org/10.1097/MAO.0b013e3181f1ce07

Yoshinaga-Itano, C., Coulter, D., \& Thomson, V. (2000). The Colorado newborn hearing screening project: Effects on speech and language development for children with hearing loss. Journal of Perinatology, 20(8, Pt. 2), S132-S137.

Yoshinaga-Itano, C., Sedey, A. L., Coulter, D. K., \& Mehl, A. L. (1998). Language of early- and later-identified children with hearing loss. Pediatrics. 102(5), 1161-1171. https://doi.org/10.1542/peds.102.5.1161

Young, A., Carr, G., Hunt, R., McCracken, W., Skipp, A., \& Tattersall, H. (2006). Informed choice and deaf children: Underpinning concepts and enduring challenges. Journal of Deaf Studies and Deaf Education, 11(3), 322-336. https://doi.org/10.1093/deafed/enj041

Young, N. M., Reilly, B. K., \& Burke, L. (2011). Limitations of universal newborn hearing screening in early identification of pediatric cochlear implant candidates. Archives of Otolaryngology-Head \& Neck Surgery, 137(3), 230-234. https://doi.org/10.1001/archoto.2011.4

Young, J. Y., Ryan, M. E., \& Young, N. M. (2014). Preoperative imaging of sensorineural hearing loss in pediatric candidates for cochlear implantation. Radiographics, 34, E133-E149. https://doi.org/10.1148/rg.345130083

Zimmerman, W., Ganzel, T., Windmill, I., Nazar, G., \& Phillips, M. (2003). Peripheral hearing loss following head trauma in children.

Laryngoscope, 103(1), 87-91. 